Linköping studies in science and technology. Thesis

No. 1808

\title{
On Timing-Based Localization in Cellular Radio Networks
}

Kamiar Radnosrati

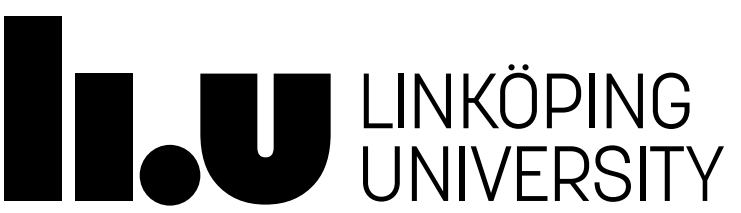


This is a Swedish Licentiate's Thesis.

Swedish postgraduate education leads to a Doctor's degree and/or a Licentiate's degree.

A Doctor's Degree comprises 240 ECTS credits (4 years of full-time studies).

A Licentiate's degree comprises 120 ECTS credits,

of which at least 60 ECTS credits constitute a Licentiate's thesis.

Linköping studies in science and technology. Thesis

No. 1808

On Timing-Based Localization in Cellular Radio Networks

Kamiar Radnosrati

kamiar.radnosratieliu.se

www. control.isy.liu.se

Department of Electrical Engineering

Linköping University

SE-581 83 Linköping

Sweden

ISBN 978-91-7685-269-9ＩSSN 0280-7971

Copyright (C) 2018 Kamiar Radnosrati

Printed by LiU-Tryck, Linköping, Sweden 2018 
To my family and friends 



\section{Abstract}

The possibilities for positioning in cellular networks has increased over time, pushed by increased needs for location based products and services for a variety of purposes. It all started with rough position estimates based on timing measurements and sector information available in the global system for mobile communication (GSM), and today there is an increased standardization effort to provide more position relevant measurements in cellular communication systems to improve on localization accuracy and availability. A first purpose of this thesis is to survey recent efforts in the area and their potential for localization. The rest of the thesis then investigates three particular aspects, where the focus is on timing measurements. How can these be combined in the best way in long term evolution (LTE), what is the potential for the new narrow-band communication links for localization, and can the timing measurement error be more accurately modeled?

The first contribution concerns a narrow-band standard in LTE intended for internet of things (IOT) devices. This LTE standard includes a special position reference signal sent synchronized by all base stations (BS) to all IOT devices. Each device can then compute several pair-wise time differences that corresponds to hyperbolic functions. Using multilateration methods the intersection of a set of such hyperbolas can be computed. An extensive performance study using a professional simulation environment with realistic user models is presented, indicating that a decent position accuracy can be achieved despite the narrow bandwidth of the channel.

The second contribution is a study of how downlink measurements in LTE can be combined. Time of flight (TOF) to the serving BS and time difference of arrival (TDOA) to the neighboring BS are used as measurements. From a geometrical perspective, the position estimation problem involves computing the intersection of a circle and hyperbolas, all with uncertain radii. We propose a fusion framework for both snapshot estimation and filtering, and evaluate with both simulated and experimental field test data. The results indicate that the position accuracy is better than 40 meters $95 \%$ of the time.

A third study in the thesis analyzes the statistical distribution of timing measurement errors in LTE systems. Three different machine learning methods are applied to the experimental data to fit Gaussian mixture distributions to the observed measurement errors. Since current positioning algorithms are mostly based on Gaussian distribution models, knowledge of a good model for the measurement errors can be used to improve the accuracy and robustness of the algorithms. The obtained results indicate that a single Gaussian distribution is not adequate to model the real TOA measurement errors. One possible future study is to further develop standard algorithms with these models. 



\section{Populärvetenskaplig sammanfattning}

Lokalisering, att bestämma sin position, i våra mobilnät är en möjlighet som skapat många nya produkter och tjänster. Det började med att man i GSM (Global System for Mobile Communication) utnyttjade en grov avståndsmätning som fanns med i första standarden, samt vilken sektorantenn användaren var ansluten till, för att få en "tårtbit" som representerade möjliga positioner. Idag har alla mobilstandarder fler mätningar som relaterar till användarens position, och nya tekniker utvecklas ständigt för att få bättre noggrannhet och tillgänglighet $\mathrm{i}$ positioneringen. Dessutom har de flesta av dagens telefoner inbyggd satellitbaserad positionering, främst GPS (Gobal Positioning System), för applikationer som har speciellt höga krav på positions-noggrannhet. GPS förbrukar dock mycket batteri-effekt, och är ingen lösning för bakgrundstjänster, som kräver tillgång till position kontinuerligt men inte har samma krav på noggrannhet. Exempel på sådana tjänster är kommunikationstjänsten i sig, som behöver veta var mobilen är och vart den är på väg för att optimera uppkopplingen.

Det som positionering har gemensamt i GPS och cellulära nätverk är behovet av noggranna tidsmätningar, samt algoritmer för att beräkna en position från flera tidsmätningar. Avhandlingen studerar ett par aspekter på tidsmätningar i nya standarder, och presenterar skräddarsydda algoritmer för positionering i dessa fall, samt utvärderar deras potential för positionering.

Den första aspekten är hur man i long term evoluion (LTE) använder tidsmätningar från den bas-station (BS) mobilen är uppkopplad mot för att räkna ut ett avstånd som svarar mot en cirkel kring denna BS. För att komplettera denna information kan mobilen också räkna ut tidsskillnader från utsända pilotsymboler från den uppkopplade BS till ett antal andra BS. Dessa svarar geometriskt mot hyperbler. Positionerings-problemet kan då formuleras som att hitta skärningen mellan en cirkel och en eller flera hyperbler. Avhandlingen beskriver ett ramverk för hur detta problem kan lösas, dels för varje mätning separat, dels som ett filtrerings-problem där användarens position följs över tiden. Resultat från fältprov visar att man i $95 \%$ av tiden får en noggrannhet som är bättre än 40 meter.

Den andra aspekten relaterar till sakernas internet (Internet of Things, IOT), där det i LTE finns en separat standard för smalbandiga kommunikationskanaler anpassade för IOT. Smalbandigheten gör IOT-enheterna batteri-effektiva, men skapar också problem för de tidsmätningar som krävs för positionering. Standarden föreskriver att speciella symboler ska skickas synkront i nerlänk från varje basstation, och IOT-enheten kan från dessa räkna ut tidsskillnader mellan parvisa BS. Dessa svarar mot hyperbler, och multilateringstekniker kan användas för att räkna ut deras skärningspunkt med tillhörande osäkerhetsmått. Avhandlingen presenterar en utförlig prestanda-studie baserad på en professionell simulator med realistiska modeller för mobilernas rörelse. Resultat är att man kan få en noggrannhet och tillgänglighet som matchar dagens metoder i cellulära nätverk, trots den mycket lägre bandbredden.

En tredje studie $\mathrm{i}$ avhandlingen är en mer generell analys av hur felet i tidsmätningar i LTE fördelar sig statistiskt. Ett antal olika metoder som är populära 
inom maskininlärning appliceras på fältdata, och mixturer av normalfördelningar anpassas till observerade mätfel. En bra modell för mätfel kan i sig förbättra alla metoder för positionering från tidsmätningar, där tumregeln är att ju mer fördelningen avviker från en normalfördelning, desto större förbättringspotential finns det i de algoritmer som används idag. Resultatet är att verkliga tidsfel fördelas sig tämligen olikt en normalfördelning, så ett en möjlig fortsättning på avhandlingen är att vidareutveckla standardalgoritmer med dessa modeller. 


\section{Acknowledgments}

Around three years ago, I started a new path as a $\mathrm{PhD}$ candidate without being sure what it would actually be like. Like any other short- or long- term goals in life, I could imagine a winding journey with its own ups and downs. Looking back on this experience, I could see that last couple of years were a mixture of challenges making me temporarily disappointed, as well as joyful moments pushing me forward. Needless to say, transitioning between the two modes would not have been as smooth without the help from many of you, and I'd like to thank everyone who has assisted me on this journey.

I would like to thank my supervisor Fredrik Gustafsson for trusting in me in the first place as a PhD student, and for offering me never-ending inspiring ideas and great support in the past three years. I am also grateful for your great advice on writing different parts of scientific reports. Sorry that you can still spot some mistakes, the "use of articles" for example. I hope that I managed to come up with an acknowledgment that does not contain many errors. I would also like to thank Svante Gunnarsson and Martin Enqvist for providing such an extraordinary work environment. Ninna Stensgård, thanks a lot for all your help with administrative issues and the large number of letters you provided to help me with different organizations and embassies.

I would like to give special thanks to the great co-supervisors I was privileged to have. No matter if it is a working day or not, Gustaf Hendeby has always been there, and would greet me with "what can I do for you?" whenever I knocked at his door. Gustaf, there is a long list of things to be thankful for. To mention a few, I could name the IATEXtemplate we all use for our theses, your helpful advice when I get stuck in research or even non work-related issues, your (handwritten) comments and corrections on the papers, etc. When it comes to the draft version of my papers or other reports, I will never forget the constructive comments given by Carsten Fritsche. I have learned a lot from the very long emails and discussions from you Carsten. Your ideas have always been inspiring. Thank you for being such a great company in the events outside the work. I would like to thank Fredrik Gunnarsson for giving me the chance to join Ericsson research in Linköping as a visiting researcher for two months and for the ideas he shared with me since the beginning of my PhD studies.

I would also like to thank the great colleagues in Automatic Control group who made an amazing atmosphere at work. I would like to say thank you to Angela Fontan, Shervin Parvini, Du Ho, Alberto Zenere, Per BostrÖm, Kristoffer Bergman and all other colleagues, better call them friends, for the great moments we have had in different occasions. As for the proofreaders of this thesis, Parinaz Kasebzadeh, Martin Lindfors, Erik Hedberg, Andreas BergstrÖm, and Yuxin Zhao.

I would like to acknowledge funding from the European Union FP7 Marie Curie training program on Tracking in Complex Sensor Systems (TRAX), grant number 607400, and the project Scalable Kalman Filters granted by the Swedish Research Council.

It would be impossible for me to tackle emotional and practical challenges 
of living abroad without having lovely close friends by my side. Luckily, I have made some great ones since my master's studies in Finland. I would like to thank you Mona, Orod, Nader, Afsaneh, and Saeid for always being there for me, from long emergency Skype calls to unplanned gatherings. I also got to know many lovely people in Sweden, like Sarah and Mohammad, that I'd like to thank you for the great moments we have shared together.

My family... I can't even formulate in words how much I love and value you! You have raised me, taught me lessons that goes beyond everything I mentioned so far, and have had my back my entire life. Mom and dad, you respected and supported all my decisions and cared about me like no one ever did. I am lucky to have kind, caring, and lovely older sister and brother. Negar and Mazyar, you never let me feel lonely in my life. I would also like to express my gratitude to my brother and sister in-laws and my two lovely nephews.

Finally, I'd like to say few words about one person missing in the list of friends I made in Finland - to you Parinaz. Not that you are not a friend, but I have a few more sentences to devote to you. Our friendship started in the first year of our master's studies. I used to be with you in different labs, partnered with you in various projects, walked with you on the streets, and danced with you at parties. Since then, you have been a colleague, a friend and a family, so you deserve all my gratitude mentioned above. Thanks for everything!

Linköping, May 2018

Kamiar Radnosrati 


\section{Contents}

Notation

xiii

1 Introduction 1

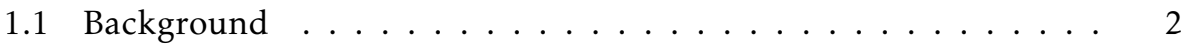

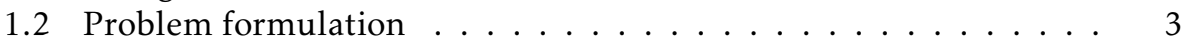

1.3 TDOA positioning in narrowband IoT systems $\ldots \ldots \ldots \ldots$

1.4 Fusion of TOF and TDOA for positioning using two BSs . . . . . 5

1.5 TOA error modeling in presence of NLOS propagation components 6

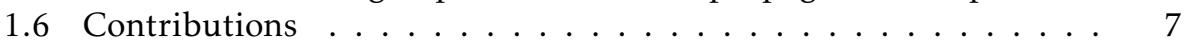

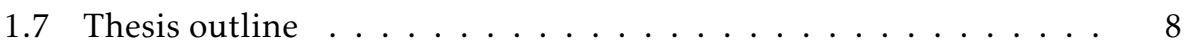

2 State of the Art in Radio Network Positioning 11

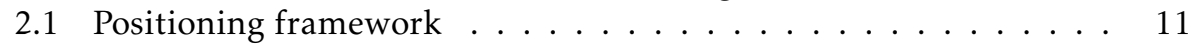

2.1.1 Level 1: Radio measurement principles . . . . . . . . 12

2.1.2 Level 2: Spatial fusion . . . . . . . . . . . . . . . . 15

2.1.3 Level 3: Modality fusion and temporal filtering . . . . . . 17

2.2 Practical considerations . . . . . . . . . . . . . . . 18

2.2 .1 Received signal strength $\ldots \ldots \ldots \ldots \ldots$

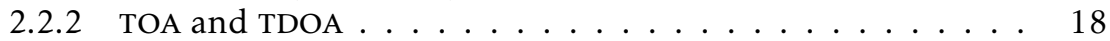

2.2 .3 Barometric pressure . . . . . . . . . . . . . . . . . 19

2.3 Trends. . . . . . . . . . . . . . . . . . . . . 20

2.3.1 New and better information . . . . . . . . . 21

2.3.2 New infrastructure . . . . . . . . . . . . . . 22

3 Theoretical Background 25

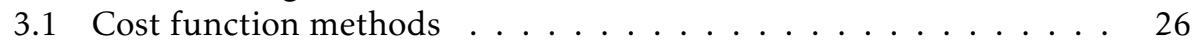

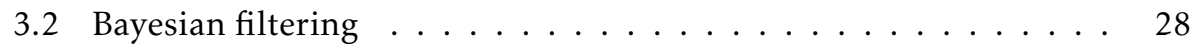

4 OTDOA Positioning in NBIOT Systems 35

4.1 Use cases of IOT positioning . . . . . . . . . . . 35

4.2 OTDOA positioning in NBIOT systems $\ldots \ldots \ldots \ldots \ldots$

4.2.1 IOT positioning in LTE standardization . . . . . . . . 36

4.2 .2 Channel model . . . . . . . . . . . . . . 38 
4.2 .3 RSTD estimation . . . . . . . . . . . . . . . 38

4.3 Simulation study . . . . . . . . . . . . . . . . . 39

4.3.1 Network deployment . . . . . . . . . . . . . . . 4 40

4.3.2 RSTD report budget .................. 40

4.3.3 Positioning scenarios and budget constraints . . . . . . . 41

4.4 Result and Conclusions . . . . . . . . . . . . . . . . . . 42

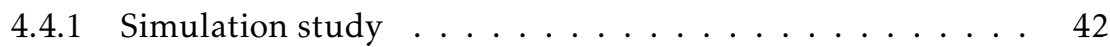

4.4 .2 Conclusions ................... 44

5 Positioning Using Fusion of TOF and TDOA 47

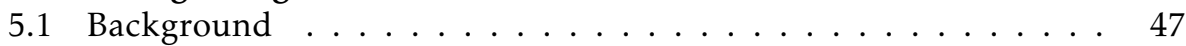

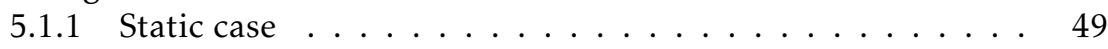

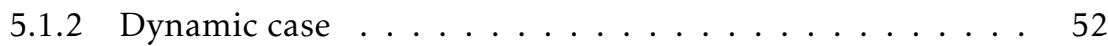

5.1.3 Lower bounds on position estimation . . . . . . . . . 58

5.2 Result and conclusion . . . . . . . . . . . . . . . 60

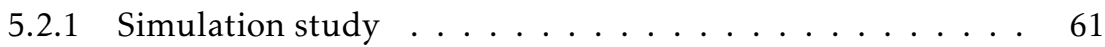

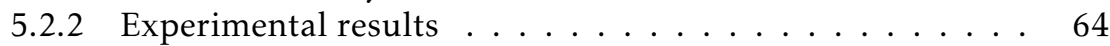

5.2 .3 Conclusions ...................... 66

6 Non-line-of-sight Error Estimation $\quad 67$

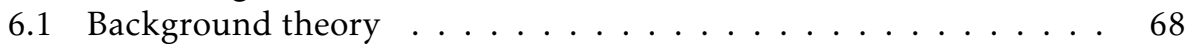

6.1.1 Gaussian mixture models ............. 68

6.1.2 Expectation maximization ............ 70

6.1 .3 Gibbs sampling ...................... 71

6.1.4 Quasi-Bayes approximation ............. 75

6.2 Performance evaluation . . . . . . . . . . . . . . 77

6.2 .1 Simulated model . . . . . . . . . . . . 78

6.2 .2 Experimental data . . . . . . . . . . . 80

7 Concluding Remarks and Future Work $\quad 87$

7.1 State of the art in radio network positioning . . . . . . . 87

7.2 Performance of OTDOA positioning in NBIOT systems . . . . . . . 88

7.3 Fusion of TDOA and TOF for positioning with two involved BSs . . 88

7.4 TOA error modeling in presence of NLOS components . . . . . . . 89

$\begin{array}{ll}\text { Bibliography } & 91\end{array}$ 
Mathematical Symbols and Operations

\begin{tabular}{cl}
\hline Notation & Meaning \\
\hline$a$ & Scalar variable \\
$\boldsymbol{a}$ & Column vector variable \\
$\boldsymbol{A}$ & Matrix variable \\
$\mathbf{0}$ & Column zero vector of appropriate size \\
$\boldsymbol{I}_{N}$ & Identity matrix of size $N \times N$ \\
{$[\cdot]^{T}$} & Vector/Matrix transpose \\
{$[\cdot]^{-1}$} & Non-singular square matrix inverse \\
$\operatorname{tr}(\cdot)$ & Trace of square matrix \\
$\|\cdot\|$ & Euclidean norm \\
$|\cdot|$ & Cardinality of a set \\
$\arg \max$ & Maximizing argument \\
$\arg \min$ & Minimizing argument \\
\hline
\end{tabular}

\section{Probability Symbols and Operations}

\begin{tabular}{cl}
\hline Notation & Meaning \\
\hline$p(\cdot)$ & Probability density function \\
$p(\cdot \mid \cdot)$ & Conditional probability density function \\
$p(\cdot ; a)$ & Probability density function parameterized by vari- \\
& able or expression a \\
$\mathbb{E}_{a}$ & Expectation with respect to stochastic variable $\boldsymbol{a}$ \\
$\operatorname{Cov}(a)$ & Covariance of the stochastic variable $\boldsymbol{a}$ \\
$\mathcal{N}(\mu, \Sigma)$ & Normal distribution with mean $\mu$ and covariance $\Sigma$ \\
\hline
\end{tabular}




\begin{tabular}{|c|c|}
\hline Abbreviation & Meaning \\
\hline 3GPP & 3rd Generation Partnership Project \\
\hline AWGN & Additive White Gaussian Noise \\
\hline BS & Base Station \\
\hline $\mathrm{CDF}$ & Cumulative Distribution Function \\
\hline $\mathrm{CP}$ & Constant Position \\
\hline CRLB & Cramér-Rao Lower Bound \\
\hline $\mathrm{CV}$ & Constant Velocity \\
\hline EKF & Extended Kalman Filter \\
\hline $\mathrm{EM}$ & Expectation Maximization \\
\hline EPA & Extended Pedestrian A \\
\hline ETU & Extended Typical Urban \\
\hline GNSS & Global Navigation Satellite System \\
\hline IOT & Internet Of Things \\
\hline JMM & Jump Markov Model \\
\hline KF & Kalman Filter \\
\hline LOS & Line Of Sight \\
\hline LTE & Long Term Evolution \\
\hline MAP & Maximum A Posteriori \\
\hline MCMC & Markov Chain Monte Carlo \\
\hline MMSE & Minimum Mean Squared Error \\
\hline NBIOT & Narrowband Internet of Things \\
\hline NLOS & Non Line Of Sight \\
\hline NPRS & Narrowband Positioning Reference Signal \\
\hline OTDOA & Observed Time Difference Of Arrival \\
\hline PDF & Probability Density Function \\
\hline PRS & Positioning Reference Signal \\
\hline QB & Quasi Bayes \\
\hline RMSE & Root Mean Square Error \\
\hline RSTD & Reference Signal Time Difference \\
\hline SSM & State-Space Model \\
\hline TDOA & Time Difference Of Arrival \\
\hline TOA & Time Of Arrival \\
\hline TOF & Time Of Flight \\
\hline UE & User Equipment \\
\hline UT & Unscented Transform \\
\hline WLS & Weighted Least Square \\
\hline
\end{tabular}




\section{1}

\section{Introduction}

Positioning capability in devices and gadgets is currently in transformation from "nice to have" to "a must". First, we have safety legislations giving tough specifications on the position information in emergency calls. Then, we have the rapid development of location based services which requires positioning in situations where satellite navigation systems do not work (indoors, underground, etc). Further, a rapidly increasing number of devices connected to the cellular network that are not operated by humans. We have the trends of Internet of Things, machine to machine communication, autonomous vehicles and systems, etc., where communication and positioning will be the key enabler for future functions and services.

While cellular radio networks were traditionally designed for communication purposes, their importance for positioning was soon realized. Awareness of the location in cellular systems is beneficial for the network operators as well as the end users. For example, such information enables network operators to manage their resource consumption, to provide location-based services, and for locationaware advertisement purposes, among others. Hence, determining the location of a source in a cellular system has been receiving considerable attention.

Different types of measurements can be defined based on the type of the measured property of the wireless communication channel. This thesis investigates the propagation time of a reference signal transmitted between the base station (BS) with a priory known location and the user equipment (UE). In the rest of this chapter, a background on timing-based localization in cellular radio networks is first presented in Section 1.1 followed by problem formulation of the work described in Section 1.2. Then, the research motivations for three particular aspects of localization with the focus on timing measurements are provided. Section 1.3 concerns observed time difference of arrival (OTDOA) localization in narrow-band internet of things (NBIOT) systems. Section 1.4 concerns fusion of time of flight 
(TOF) and time difference of arrival (TDOA) in long term evolution (LTE) systems using measurements collected from two BSs. Section 1.5 concerns a more general analysis of how the error in time measurements in LTE is distributed statistically. Finally, the author's publications and contributions are presented in Section 1.6 followed by the outline of this thesis provided in Section 1.7.

\subsection{Background}

The positioning accuracy in the early stages of realizing the potential of cellular systems for positioning was rather poor which was due to the fact that the used signals were not designed for positioning purposes. However, in recent years there has been a tremendous standardization effort to increase this accuracy, which was also a result of federal communications commission (FCC) regulations on emergency calls that were established in the U.S [39].

Dedicated solutions such as global navigation satellite system (GNSS) have been, and are being, used by end users for a long time. However, mutual benefits of more reliable, yet accurate, source of information for users and cellular network operators emerged a new research direction; combining positioning and communication systems into a single system.

Timing-based positioning in cellular systems is based on time of arrival (TOA), time of flight (TOF), and time difference of arrival (TDOA) measurements. TOA is estimated by cross-correlating the received signal with a replica of the transmitted signal waveform. Both TOF and TDOA are based on time of arrival (TOA) measurements at the UE as well as the BS. TOA is used to estimate TOF by combining TOA estimated at BS and UE, while TDOA is estimated using TOA associated to two different BSs. It might be worth mentioning that TOA requires accurate synchronization between UE and BS whereas TDOA only requires synchronization between BSs.

Timing measurements can be translated to absolute, for TOF and TOA, or relative, for TDOA, distances between the UE and the set of BSs. Knowing the location of each BS, it is possible to estimate the unknown UE position using different techniques. Closed-form solutions for hyperbolic positioning can be found for instance in [24, 50, 115]. Iterative algorithms for solving a nonlinear weighted least squares form another major group. The Gauss-Newton algorithm is studied in [22], constrained and unconstrained NLS solutions are discussed in [23, 28]. The iterative approaches generally require good initialization to converge to the global optimum of the cost function and often require many iterations. In order to avoid these issues, the solutions proposed in $[30,61]$ transform nonlinear equations into a set of linear ones, thus making real-time implementations possible. Factor graph-based methods carrying low-complex flags also attracted some attention $[26,92]$.

Starting from Release 9, LTE integrated positioning reference signals into their standard. Since then, it can be seen as a continuous trend in all consecutive updates of 3GPP standards to evolve different aspects of positioning. Localization in LTE systems is a mature research area. For example, [90] uses real TDOA mea- 
surements and investigates channel impacts on positioning accuracy. An error analysis of TDOA is reported in [74]. Baseline performance based on 3GPP 3D MIMO deployment and propagation model is investigated in [106]. The work in [81] addresses the main requirement for accurate TDOA positioning, synchronization of anchor nodes. Finally, surveys on the obtainable accuracy bounds are reported in [55], [106], and [99].

The localization accuracy, in all timing-based methods, is influenced by the accuracy of the estimated TOAs and the number of measured BSs, among others. In line of sight (LOS) environments TOA can be estimated with fairly good accuracy in LTE systems. In non-line-of-sight (NLOS) environments, however, more sophisticated algorithms are required. Modeling the observed time measurement error is widely studied in the literature to mitigate NLOS effects.

NLOS errors are modeled as a deterministic variable or as a random variable in [27]. In the former, at a fixed time instance, NLOS errors are treated as a constant variable. It is mentioned that NLOS errors depend on the propagation environment, hence their values are allowed to vary between $100 \mathrm{~m}$ to $1300 \mathrm{~m}$ in their simulations. In the second case in which NLOS errors are modeled as a random variable, it is mentioned that the errors can be modeled using an exponential, a uniform, or a delta random variable. Authors in [88] also model NLOS errors as random variables with an exponential PDF.

Empirical analysis of the real data is performed in [62] to determine the error PDF of timing-based position estimator. Authors in [62] have modeled the LOS errors with a zero-mean Gaussian whose variance depends on the SNR of the received signal. The NLOS errors are modeled with a Rayleigh distribution that should be parameterized depending on the propagation scenario. Using the Rayleigh distribution for modeling NLOS errors is also mentioned in [130].

The introduced timing measurement error models in [55] assume that LOS errors belong to a zero-mean Gaussian distribution while NLOS belong to a shifted Gaussian distribution. The authors in $[46,47,57,82]$ also model NLOs errors using shifted Gaussian densities and introduce robust timing-based position estimation methods. In [58], the second component in the mixture distribution corresponding to the NLOS errors is modeled using the convolution of the probability distribution function (PDF) of a positive random variable and the zeromean Gaussian density of LOS errors. The authors in [31] consider the Gaussiandistributed NLOS error mitigation problem and consider three different cases in which NLOS are assumed to have known statistics, limited prior information, or totally unknown statistics.

\subsection{Problem formulation}

Positioning in cellular networks is often based on indirect observations of the user equipment's (UE's) position, measured from various properties of the wireless communication channel between base stations (BSs) and the UE. The measurements collected from a set of BSs are then further processed to infer the unknown position. In cellular radio networks, the UE is generally assigned to a specific 
BS, the serving BS, which is responsible for the communication link with the UE; other BSs are referred to as neighboring BSs.

Today's cellular radio network standards enable the configuration of positioning reference signals (PRS) from BSs which enable UE to estimate TOA measurements. In third generation partnership project (3GPP) LTE, PRS can be defined based on orthogonal patterns, as well as muting schemes, where some BSs transmit a PRS, while other BSs are muted, in order to suppress interference and ensure a wide detectability of signals.

OTDOA is a downlink positioning method in LTE systems based on PRS transmitted by the BS. While OTDOA-based positioning is widely studied in the literature, the performance evaluation for the newly released NBIOT systems is not treated with the same level of detail. One objective of this thesis is to evaluate the positioning performance in NBIOT systems. The downlink TDOA measurements estimated from narrowband positioning reference signals are used in the evaluations.

In LTE systems, positioning is traditionally considered to be enabled, in $2 \mathrm{D}$ coordinates, when the UE provides measurements of at least three different BSs. All the methods introduced in Section 1.1 are based on this requirement. In this thesis, we additionally investigate positioning based on time series of timing measurements gathered from two BSs with known positions. The set of two measured BSs is different along the trajectory. Each report contains TOF for the serving base station, and TDOA measurement for the most favorable neighboring BS relative the serving BS.

In this thesis, we also report preliminary results for the problem of modeling TOA measurement errors in presence of NLOS propagation error components. Three main sources of TOA estimation errors are [14]:

\section{Measurement noise.}

2. Multipath conditions resulting in paths that arrive close to the direct path.

3. Undetected direct path in which the direct path is blocked, hence the first arriving path is erroneously detected as the direct path.

Measurement noises have a zero mean value while the multipath error has a random but small mean value, [13] and [12]. The undetected direct path, on the other hand, is larger than the two others and has a positive mean.

In case of single path propagation, TOA measurement errors, $\boldsymbol{e}$, are modeled using a two-component mixture PDF. The first component corresponds to the LOS path and is modeled using a zero-mean Gaussian distribution $\boldsymbol{e}_{\mathrm{LOS}} \sim \mathcal{N}\left(\mathbf{0}, \sigma_{\mathrm{LOS}}^{2}\right)$. The second component, models NLOS propagation effects whose PDF is given by $\mathcal{H}(\boldsymbol{e})$. It must be noted that at each time instance, only one component of the Gaussian mixture is effective. As discussed in [131], $\mathcal{H}(\boldsymbol{e})$ has a positive bias with a variance larger than $\sigma_{\mathrm{LOS}}^{2}$. 


\subsection{TDOA positioning in narrowband lOT systems}

Uplink TDOA (UTDOA) and downlink OTDOA methods rely on different reference signals and are performed at the BS and at the UE, respectively. OTDOA, defined in 3GPP Rel-9, is a UE-assisted position measurement of TDOA in which the UE measures the TOA of signals received from multiple BSs. Then, the computed TOAs of several BSs are subtracted from the TOA of the received signal from a reference BS to form OTDOA.

TDOA positioning, in general, using different reference signals in LTE systems is a well-studied research area. However, performance analysis of positioning in NBIOT systems is limited. NBIOT is an emerging cellular technology designed to target low-cost devices, high coverage, long device battery life (more than ten years), and massive capacity.

The potential of device tracking in NBIOT systems using OTDOA measurements is evaluated in Chapter 4 . The reference signals from the serving and a number of neighboring BSs are used by the UE to estimate reference signal time differences and report them back to the network to estimate the position. The effect of the number of such reports and their reporting schemes on the horizontal positioning accuracy is also evaluated.

Three different alternatives of deploying NBIOT, namely in band, guard band, and stand alone are presented in Figure 1.1. This study simulates the deployment of NBIOT within the LTE spectrum allocation, inside the LTE carrier. The extended pedestrian A (EPA) and extended typical urban (ETU) channel models are used to account for multipath propagation effects.
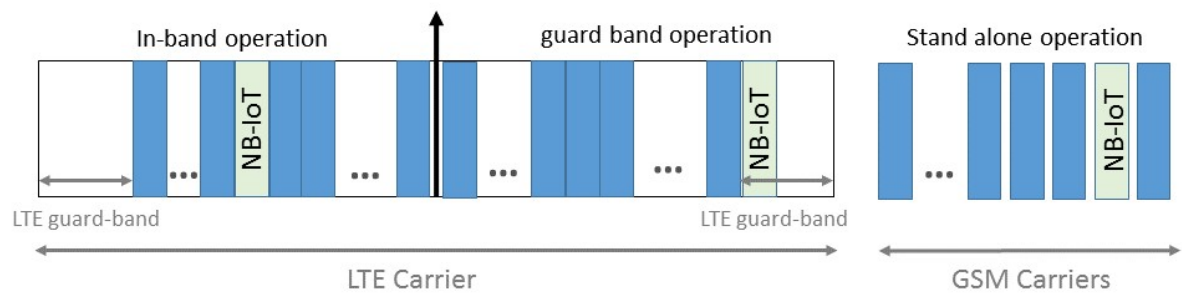

Figure 1.1: Deployment configurations of NBIOT [101, C2017 IEEE].

\subsection{Fusion of TOF and TDOA for positioning using two BSS}

UE measurements, collected from at least three base stations, are mainly used for positioning in cellular networks. To better illustrate the problem of fusing one TOF and one TDOA while three BSs are detectable, assume that BSs are deployed in a cellular radio network consisting of hexagonal cells [106] as shown in Figure 1.2. The serving BS $S_{1}$ is assumed to provide the TOF measurement, 
and two neighboring BSs $S_{2}$ and $S_{3}$ are detected by the UE to form TDOA measurements. We consider the problem in two-dimensional scenarios, and convert TOF and TDOA measurements to corresponding range and range differences. Geometrically, this means that the TOF measurement can be represented by a circle around the serving BS and the TDOA by a hyperbola with foci equivalent to the two neighboring BSs as depicted in Figure 1.2. The UE positioning problem then becomes a classical circle and hyperbola intersection problem.

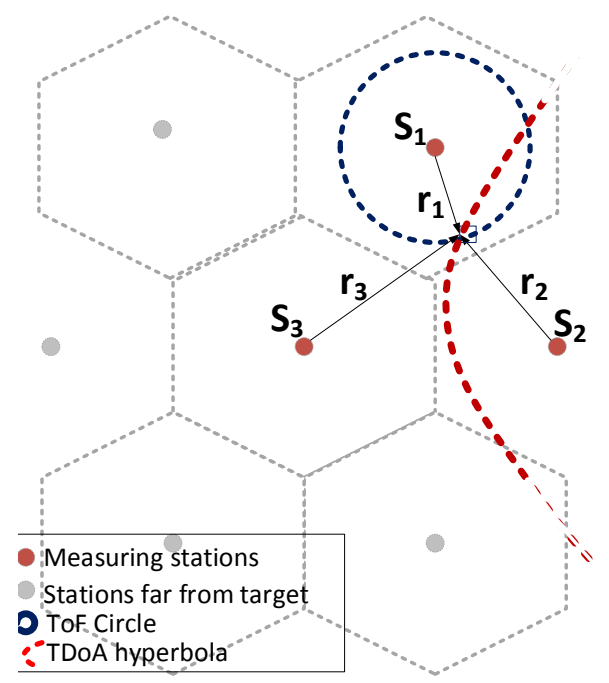

Figure 1.2: Circle of TOF measurement reported by $S_{1}$, marked with blue, and hyperbola of TDOA measurements based on the relative distance of $S_{2}$ and $S_{3}$, marked with red [100, C2016 IEEE].

This work considers a more limited case, with a time series of measurements associated to two base stations - TOF from the serving base station and TDOA measurements associated to the serving and one non-serving base station. In such cases, it is not possible to uniquely estimate the position of the UE using existing methods. Hence, more sophisticated approaches are required to deal with the ambiguity in the problem. Chapter 5 evaluates the performance of a solution based on a bank of Kalman filters.

\subsection{TOA error modeling in presence of NLOS propagation components}

All wireless positioning methods have one shared source of error, in addition to measurement noises, coming from propagation effects of the communication channel. Multipath fading, shadowing, interference, and NLOS are examples of additional errors caused by signal propagation through the wireless channel. 
To deal with performance degradation in NLOS conditions, conventional position estimation techniques which are developed based on LOS conditions need to be adopted. As discussed in [131], "identify and discard", "mathematical programming", and "robust estimation" are the three broad categories of timingbased position estimation methods which are robust against NLOS errors. In this work, we set the first stage towards developing robust estimation methods by first modeling the TOA error PDF in terms of a mixture of Gaussian distributions. The goal at this stage is to compare a deterministic, a pure Bayesian, and a quasiBayesian approach and evaluate their performances.

\subsection{Contributions}

The main contributions of this thesis can be listed as follows:

1. A survey on the state of the art in radio-based positioning, see Chapter 2 . This material is based on:

K. Radnosrati, F. Gunnarsson, F. Gustafsson. New Trends in Radio Network Positioning. In Proceedings of the 18th International Conference on Information Fusion (FUSION), Washington DC, USA, July 2015.

2. Simulation of NBIOT system and evaluation of the OTDOA positioning using simulated data, see Chapter 4 . This material has been published in:

K. Radnosrati, G. Hendeby, C. Fritsche, F. Gunnarsson, F. Gustafsson. Performance Evaluation of OTDOA Positioning in NB-IOT Systems. In Proceedings of the 28th IEEE International Symposium on Personal, Indoor and Mobile Radio Communications (PIMRC), Montreal, QC, Canada, October 2017.

3. Derivation of a closed-form solution to the problem of fusing noise-free TOF and TDOA measurements collected from two base stations. The two possible positions of the UE are then estimated using Gaussian transformation of the derived closed-form solutions. Using the two estimated positions, a jump Markov model is formulated that can handle the ambiguity in positions using extra information available at handover time instances, see Chapter 5. This material has been published in parts in:

K. Radnosrati, C. Fritsche, G. Hendeby, F. Gunnarsson, F. Gustafsson. Fusion of TOF and TDOA for 3GPP Positioning. In Proceedings of the 19th International Conference on Information Fusion (FUSION), Heidelberg, Germany, July 2016.

An extension of the work, including the extra material presented in Chapter 5, has been prepared and will be submitted to IEEE Transactions on Vehicular Technology: 
K. Radnosrati, C. Fritsche, G. Hendeby, F. Gunnarsson, F. Gustafsson. Fusion of TOF and TDOA for Timing-Based Localization. To be submitted to IEEE Transactions on Vehicular Technology.

4. Initial modeling of TOA measurement errors using the experimental data, see Chapter 6. This contribution is to be extended and is not previously published.

The content of all publications is reused in this thesis courtesy of IEEE.

\subsection{Thesis outline}

Chapter 2 presents a general positioning framework in which three main levels of information flow in positioning systems are first highlighted. In the lowest level, principles of radio measurements are introduced followed by position estimation using spatial fusion of the collected measurements in the second level. The third level consists of modality fusion and temporal filtering in state-space frameworks. Some practical considerations in radio network positioning are then discussed followed by discussions on the existing trends towards more accurate positioning systems.

Chapter 3 further discusses the last two levels introduced in Chapter 2 and provides theoretical background of position estimation methods in static and dynamic systems. In the static case, cost function methods are discussed and two well-known numerical optimization algorithms, Gauss-Newton and steepest descent, are introduced. Then, linear and nonlinear dynamic systems are briefly described and recursive state estimation in the Kalman filtering framework is discussed.

Chapter 4 evaluates positioning performance in NBIOT systems using the observed TDOA measurements. The research is motivated by the immense number of use cases of IOT positioning as briefly described. The OTDOA positioning method uses the UE estimation of the relative distance between a reference base station and a number of neighboring base stations. The estimated reference signal time differences are then reported by the UE to a positioning center to estimate the unknown location of the UE. The possibility of optimizing the number of such reports while maintaining the final horizontal position estimation accuracy within an acceptable range in a simulated network is investigated.

Chapter 5 evaluates the performance of hybrid TOF and TDOA positioning in LTE systems when only two base stations are detected by the UE. The two analytical solutions to the intersection of TOF circle and TDOA hyperbola is derived. To deal with the ambiguity caused by multiple solutions, a multimodal jump Markov system is introduced in which each mode of the system contains a possible position of the UE. A bank of Kalman filters is employed to keep track of all modes of the system until the set of involved BSs changes. The extra information obtained from this change is used to keep the true mode sequence and discard the rest. The lower bound on the achievable accuracy using the proposed method is introduced. The proposed algorithm is evaluated using both simulated and real data and the results are reported. 
Chapter 6 contains results of modeling TOA errors in presence of NLOS components. The expectation maximization (EM) and Gibbs sampling for Gaussian mixture parameter estimation together with a quasi-Bayesian approach are introduced. The performance of all three methods is then evaluated using both a simulation study and on the TOA error dataset.

Finally, Chapter 7 provides some concluding remarks and possible future research directions. 



\section{2}

\section{State of the Art in Radio Network Positioning}

Positioning in wireless networks is based on the measurements collected either at the UE and reported to the network, at the BS, or a combination thereof. All the measurements, despite the large variety of positioning systems, are essentially either based on identity labels of involved BSs, commonly referred to as cell identity, or properties of the communication link between the UE and BS. The positioning is then either based on snapshot measurements or a time series of measurements. The survey research articles $[23,36,55,109,117,135]$ report extensive information about wireless network positioning together with their associated accuracies. This chapter describes the information flow of current positioning algorithms and discusses existing trends aiming to enhance the achievable accuracy.

Section 2.1 introduces a generic measurement model and describes a typical positioning framework consisting of three different layers from the received observation to the final position. The generic measurement model is then further explained and different types of available measurements in radio networks are introduced and their associated accuracies are provided in Section 2.2. Finally, current trends aiming to improve the achievable accuracy in the presented framework are given in Section 2.3.

\subsection{Positioning framework}

The information flow in current positioning algorithms can be categorized in different levels as presented in Figure 2.1. Throughout this thesis, both the UE and the involved reference points are restricted to two dimensional scenarios. Let $\theta_{t}=\left(\theta_{x_{t}}, \theta_{y_{t}}\right)^{T}$ denote the unknown position of the UE at time $t$ and $\boldsymbol{\ell}_{t}^{(i)}=\left(\ell_{x_{t}}^{(i)}, \ell_{y_{t}}^{(i)}\right)$ denote the known position of the reference point $i$.

The generic measurement $y_{t}^{(i)}$ relative to the reference point $i$ at time $t$ is a 


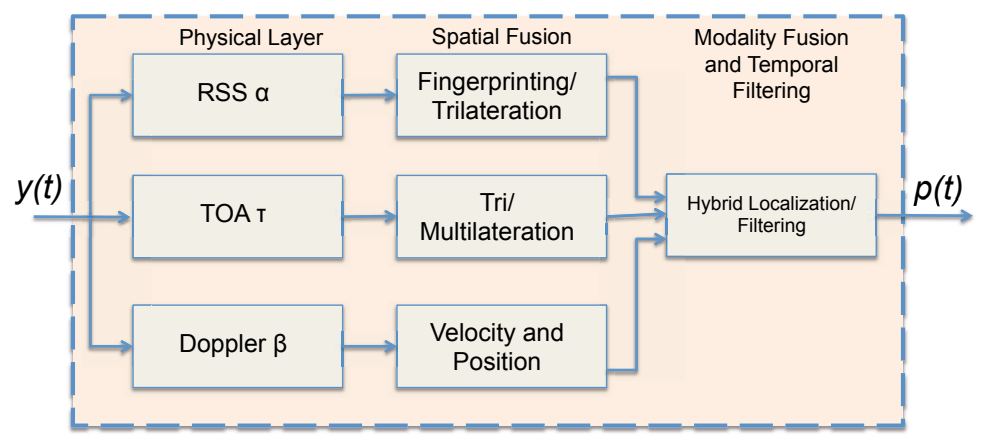

Figure 2.1: Levels of Information fusion for radio network positioning [99, C2015 IEEE].

function of both $\boldsymbol{\theta}_{t}$ and $\boldsymbol{\ell}_{t}^{(i)}$, subject to measurement noise $e_{t}^{(i)}$. Under additive measurement noise assumption, the generic model is given by

$$
y_{t}^{(i)}=h_{t}\left(\boldsymbol{\theta}_{t}, \boldsymbol{\ell}_{t}^{(i)}\right)+e_{t}^{(i)}
$$

The measurement model (2.1) is in the most generic form where the reference points can also move in time, as in some ad-hoc network problems. However, in case of snapshot measurements, or time series of measurements with fixed reference points, the time subscripts may be ignored.

\subsubsection{Level 1: Radio measurement principles}

Radio measurement, in the lowest layer of the system, is based on the received pilot signal which is transmitted over the communication channel for different purposes including referencing. The transmitted pilot symbol $s^{(i)}(t)$, in the physical layer, is sampled at the receiver

$$
z^{(i)}(t)=\sum_{k=0}^{n} \alpha_{k}^{(i)} s\left(\beta_{k}^{(i)}\left(t-\tau_{k}^{(i)}\right)\right)+e_{k}^{(i)}(t)
$$

where $\alpha_{k}^{(i)}$ is the impulse response of the multi-path channel, $\tau_{k}^{(i)}$ is the time delay per incoming path, and $\beta_{k}^{(i)}$ is the Doppler shift that scales time. Assuming that the receiver can estimate these parameters, different higher layer position-related measurements can be defined based on the parameters $\alpha, \tau$, or $\beta$ as described in the following. The generic function $h(\cdot)$ introduced in (2.1) can then be defined for each position-related measurement.

\section{Measurements based on $\tau$}

Three different higher layer measurements can be defined corresponding to $\tau_{k}^{(i)}$ : 
1. Time of Arrival corresponds to the absolute distance between the emitting and receiving nodes using the travel time of the signal transmitted between the two

$$
y_{t}^{(i), \mathrm{TOA}}=\frac{1}{c}\left\|\boldsymbol{\theta}_{t}-\boldsymbol{\ell}_{t}^{(i)}\right\|+e_{t}^{(i), \mathrm{TOA}}
$$

where $c$ is the speed of radio waves and the measurement error $e_{t}^{(i), \text { TOA }}$ captures both the estimation error and the model error due to multipath assuming that the emitter and receiver are perfectly synchronized. Otherwise, an additional error emerges from the clock offset between transceivers.

2. Time Difference of Arrival is the timing difference between two TOA measurements estimated from signals that are sent at the same time. This yields

$$
y_{t}^{(i j), \mathrm{TDOA}}=\frac{1}{c}\left\|\boldsymbol{\theta}_{t}-\boldsymbol{\ell}_{t}^{(i)}\right\|-\frac{1}{c}\left\|\boldsymbol{\theta}_{t}-\boldsymbol{\ell}_{t}^{(j)}\right\|+e_{t}^{(i), \mathrm{TOA}}-e_{t}^{(j), \mathrm{TOA}} .
$$

TDOA measurements can be obtained in both uplink and downlink directions. In the former, the UE transmits a signal to a pair of receiving BSs, hence the network is responsible for estimating the uplink TDOA. In the downlink mode, a pair of BSs will instead send reference signals to the receiving UE that is responsible for estimating the observed TDOA, known as OTDOA. Since the emission time of the signal is exactly the same, the synchronization between receiver and transmitter is no longer required. Instead, in both cases, the involved BSs need to be synchronized.

3. Time of Flight ${ }^{1}$ corresponds to the sum of the TOA measurements in both uplink and downlink directions. Figure 2.2 illustrates how TOF is estimated in LTE systems. In LTE, $T_{s} \approx 32 \mathrm{~ns}$ is the basic time unit [6], hence only $N_{T}$, in steps of $16 T_{s}$, depends on the channel quality and is updated by the network.

At the uplink transmission time $T x_{\mathrm{UL}}$, the UE transmits either a random access or demodulation reference signal and the BS measures the uplink TOA $\left(\mathrm{TOA}_{\mathrm{UL}}\right)$. The BS then sends a first $N_{T}$ to the UE to be used when deciding when to send the next uplink transmission in relation to the downlin TOA $\left(\mathrm{TOA}_{D L}\right)$. For subsequent uplink transmissions, the BS regularly sends relative corrections to $N_{T}$ in steps of $16 \mathrm{Ts}$ which means that the UE as well as the network maintains an updated $N_{T}$. In addition, the BS tries to match a certain arrival time of uplink signals in relation to the downlink transmission time (start of DL frame), $T x_{D L}$, and this is represented by $\Delta T$. The TOF measurement is thus given by,

$$
\begin{aligned}
y_{t}^{(i), \mathrm{TOF}} & =N_{T} T_{s}-\Delta T+e_{t}^{(i), \mathrm{TOF}} \\
& =\frac{2}{c}\left\|\boldsymbol{\theta}_{t}-\boldsymbol{\ell}_{t}^{(i)}\right\|+e_{t}^{(i), \mathrm{TOA}_{\mathrm{DL}}}+e_{t}^{(i), \mathrm{TOA}_{\mathrm{UL}}} .
\end{aligned}
$$

\footnotetext{
1 or round trip time.
} 


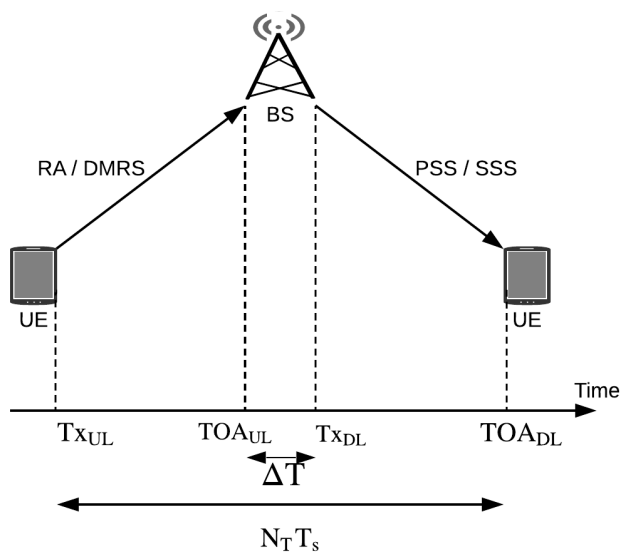

Figure 2.2: TOF measurement in LTE systems. In the uplink, random access (RA) or demodulation reference signal (DMRS) is transmitted. In the downlink, primary synchronization signal (PSS) or secondary synchronization signal (SSS) is transmitted.

4. Angle of arrival (AOA) can be computed by comparing delays $\tau$ of the received signal to multiple antennas or by using directional antennas. The high-level measurement is

$$
y_{t}^{(i), \mathrm{AOA}}=\arctan \left(\theta_{y_{t}}-\ell_{y_{t}}^{(i)}, \theta_{x_{t}}-\ell_{x_{t}}^{(i)}\right)+e_{t}^{(i), \mathrm{AOA}}
$$

The angle of the received signal could either be computed using directional antennas in which the main drawback is implementation cost of such antennas, if their sizes need to be rather small. Using an array of antennas is yet another alternative in which AOA is inferred indirectly from TOA measurement. Sophisticated algorithms are defined for array processing problems, see [75]. Additionally, AOA estimation can be performed using the antenna lobe diagram, see [53] for example.

\section{Measurements based on $\alpha$}

Received signal strength (RSS) is a ranging measurement that corresponds to the total energy of the received signal, $\sum_{k=0}^{n} \alpha_{i, k}^{2}$. The generic model for RSs measurement is given by

$$
y_{t}^{(i), \operatorname{RSS}}=h_{\mathrm{RSS}}\left(\left\|\boldsymbol{\theta}_{t}-\boldsymbol{\ell}^{(i)}\right\|\right)+e_{t}^{(i), \mathrm{RSS}},
$$

where $h_{\mathrm{RSS}}\left(\left|\boldsymbol{\theta}_{t}-\boldsymbol{\ell}^{(i)}\right|\right)$ is a deterministic function denoting the received signal strength due to path loss. Let $P_{0}^{(i)}$ denote the measured RSS of the $i$ th $\mathrm{BS}$ at a 
reference distance $d_{0}$. The deterministic function for RSS due to path loss can be written as:

$$
h_{\mathrm{RSS}}\left(\left\|\boldsymbol{\theta}_{t}-\boldsymbol{\ell}^{(i)}\right\|\right)=P_{0}^{(i)}+10 \eta \log \left(\frac{\left\|\boldsymbol{\theta}_{t}-\boldsymbol{\ell}_{t}^{(i)}\right\|}{d_{0}}\right),
$$

where $\eta$ is the path-loss exponent.

\section{Measurements based on $\beta$}

The estimated parameter $\beta$ can be interpreted as a measure of the relative speed between the UE and BS. Thus the measurement model is

$$
y_{t}^{(i), \text { Doppler }}=\frac{\partial\left\|\theta_{t}-\boldsymbol{\ell}_{t}\right\|}{\partial t}+e_{t}^{(i), \text { Doppler }} .
$$

\subsubsection{Level 2: Spatial fusion}

The information obtained from multiple, spatially distributed, sensors is fused at the second level. Let $N$ denote the number of transmitters from which measurements corresponding to the ones introduced in Section 2.1.1 are obtained. The set of equations are given by

$$
y_{t}^{(i), \text { type }}=h^{\text {type }}\left(\left\|\theta_{t}-\boldsymbol{\ell}_{t}^{(i)}\right\|\right)+e_{t}^{(i), \text { type }}, \quad i=1, \ldots, N
$$

where type is either TOA, TDOA, AOA, RSS, or Doppler. Basic methods of position estimation using the first four types of measurements are briefly explained in the remainder of this section, while more advanced methods using timing measurements are introduced and applied in the later chapters.

It must be noted that in addition to the wireless positioning methods other alternatives also exist. For instance there are some frameworks that do not use wireless communication infrastructures but rather depend on, for example, image processing techniques or dead reckoning approaches [69]. To maintain the focus of this thesis they are not discussed.

Using the range or angle measurements, the known position of BSs, and the trigonometry properties, it is possible to estimate the unknown position of the UE. Since no temporal dependency is considered in these methods, and to simplify the notation, the time subscript $t$ is dropped in the derivations. Additionally, the measurement noises of $N$ involved BSs are assumed to be normally distributed with zero mean and covariance $\boldsymbol{R}$ i.e., $\boldsymbol{e} \sim \mathcal{N}\left(\mathbf{0}, \boldsymbol{R}_{N \times N}\right)$. In this Section, we use different tricks to linearize the system to obtain the matrices $\boldsymbol{H}_{N \times 2}$ and $\boldsymbol{Y}_{N \times 1}$ such that $\boldsymbol{Y}=\boldsymbol{H} \boldsymbol{\theta}+\boldsymbol{e}$. Thus, $\hat{\boldsymbol{\theta}}$ can be computed as weighted least squares (WLS) estimator, $\hat{\boldsymbol{\theta}}=\left(\boldsymbol{H}^{T} \boldsymbol{W} \boldsymbol{H}\right)^{-1} \boldsymbol{H}^{T} \boldsymbol{W} \boldsymbol{Y}$ where the weighting matrix $\boldsymbol{W}=\boldsymbol{R}^{-1}$. The only difference is how the $\boldsymbol{H}$ and $\boldsymbol{Y}$ are formed using either of TOA, TDOA, or AOA measurements. In the following, different methods are briefly introduced, see [45] for more details. 
TOA

The absolute distances between the UE and measured BSs are used in lateration techniques to localize the UE. In a noise-free situation, the TOA circles of $N \geq 3$ BSs intersect in a single location in 2-D. However, in case of noisy measurements, the circles do not intersect in a single point and thus data fusion techniques are required to estimate the best possible position. In order to combine the available observations collected from $N$ BSs, and to linearize the equations, one trick is to subtract the distances between the UE and $\mathrm{BSs}^{(i)}, i=2, \ldots, N$ from a reference $\mathrm{BS}^{(1)}$. Let $r_{i}=y^{(i), \mathrm{TOA}}$, as shown in [45], expanding (2.3) gives

$$
r_{i}^{2}-r_{1}^{2}=\left\|\boldsymbol{\ell}^{(i)}\right\|^{2}-\left\|\boldsymbol{\ell}^{(1)}\right\|^{2}-2 \theta_{x}\left(\ell_{x}^{(i)}-\ell_{x}^{(1)}\right)-2 \theta_{y}\left(\ell_{y}^{(i)}-\ell_{y}^{(1)}\right),
$$

the matrices are thus given by

$$
\begin{gathered}
\boldsymbol{H}=\left[\begin{array}{cc}
\ell_{x}^{(2)}-\ell_{x}^{(1)} & \ell_{y}^{(2)}-\ell_{y}^{(1)} \\
\ell_{x}^{(3)}-\ell_{x}^{(1)} & \ell_{y}^{(3)}-\ell_{y}^{(1)} \\
\vdots & \vdots \\
\ell_{x}^{(N)}-\ell_{x}^{(1)} & \ell_{y}^{(N)}-\ell_{y}^{(1)}
\end{array}\right], \\
\boldsymbol{Y}=\frac{1}{2}\left[\begin{array}{c}
r_{1}^{2}-r_{2}^{2}+\left\|\boldsymbol{\ell}^{(2)}\right\|^{2}-\left\|\boldsymbol{\ell}^{(1)}\right\|^{2} \\
r_{1}^{2}-r_{3}^{2}+\left\|\boldsymbol{\ell}^{(3)}\right\|^{2}-\left\|\boldsymbol{\ell}^{(1)}\right\|^{2} \\
\vdots \\
r_{1}^{2}-r_{N}^{2}+\left\|\boldsymbol{\ell}^{(N)}\right\|^{2}-\left\|\boldsymbol{\ell}^{(1)}\right\|^{2}
\end{array}\right] .
\end{gathered}
$$

TDOA

To localize the UE using relative distances given by TDOA measurements, hyperbolic localization techniques can be used. Using the same notation as in lateration, the relative distances $r_{i 1}=r_{i}-r_{1}$. Following the method introduced in [45], one can get

$$
r_{i 1}^{2}+2 r_{i 1} r_{1}=r_{i}^{2}-r_{1}^{2}
$$

that can be expanded as

$$
r_{i 1}^{2}+2 r_{i 1} r_{1}=\left\|\boldsymbol{\ell}^{(i)}\right\|^{2}-\left\|\boldsymbol{\ell}^{(1)}\right\|^{2}-2 \theta_{x}\left(\ell_{x}^{(i)}-\ell_{x}^{(1)}\right)-2 \theta_{y}\left(\ell_{y}^{(i)}-\ell_{y}^{(1)}\right) .
$$


Since the TOA measurement $r_{1}$ is unknown, it should be added to the parameter vector as well. Thus, $\tilde{\boldsymbol{\theta}}=\left(\theta_{x}, \theta_{y}, r_{1}\right)^{T}$ and we solve $\boldsymbol{Y}=\boldsymbol{H} \tilde{\boldsymbol{\theta}}$ for $\tilde{\boldsymbol{\theta}}$ where

$$
\begin{gathered}
\boldsymbol{H}=\left[\begin{array}{ccc}
\ell_{x}^{(2)}-\ell_{x}^{(1)} & \ell_{y}^{(2)}-\ell_{y}^{(1)} & r_{21} \\
\ell_{x}^{(3)}-\ell_{x}^{(1)} & \ell_{y}^{(3)}-\ell_{y}^{(1)} & r_{31} \\
\vdots & \vdots & \vdots \\
\ell_{x}^{(N)}-\ell_{x}^{(1)} & \ell_{y}^{(N)}-\ell_{y}^{(1)} & r_{N 1}
\end{array}\right], \\
\boldsymbol{Y}=\frac{1}{2}\left[\begin{array}{c}
\left\|\boldsymbol{\ell}^{(2)}\right\|^{2}-\left\|\boldsymbol{\ell}^{(1)}\right\|^{2}-r_{21}^{2} \\
\left\|\boldsymbol{\ell}^{(3)}\right\|^{2}-\left\|\boldsymbol{\ell}^{(1)}\right\|^{2}-r_{31}^{2} \\
\vdots \\
\left\|\boldsymbol{\ell}^{(N)}\right\|^{2}-\left\|\boldsymbol{\ell}^{(1)}\right\|^{2}-r_{N 1}^{2}
\end{array}\right] .
\end{gathered}
$$

AOA

The position of the UE can be estimated from AOA measurements using angulation technique. Let $\alpha_{i}$ denote the measured angle of the received signal transmitted by the $\mathrm{BS}^{(i)}$. As discussed in [45], equation (2.6) gives

$$
\left(\ell_{x}^{(i)}-\theta_{x}\right) \sin \left(\alpha_{i}\right)=\left(\ell_{y}^{(i)}-\theta_{y}\right) \cos \left(\alpha_{i}\right)
$$

with

$$
\begin{gathered}
\boldsymbol{H}=\left[\begin{array}{cc}
-\sin \left(\alpha_{1}\right) & \cos \left(\alpha_{1}\right) \\
-\sin \left(\alpha_{2}\right) & \cos \left(\alpha_{2}\right) \\
\vdots & \vdots \\
-\sin \left(\alpha_{N}\right) & \cos \left(\alpha_{N}\right)
\end{array}\right], \\
\boldsymbol{Y}=\frac{1}{2}\left[\begin{array}{c}
\ell_{y}^{(1)} \cos \left(\alpha_{1}\right)-\ell_{x}^{(1)} \sin \left(\alpha_{1}\right) \\
\ell_{y}^{(2)} \cos \left(\alpha_{2}\right)-\ell_{x}^{(2)} \sin \left(\alpha_{2}\right) \\
\vdots \\
\ell_{y}^{(N)} \cos \left(\alpha_{N}\right)-\ell_{x}^{(N)} \sin \left(\alpha_{N}\right)
\end{array}\right] .
\end{gathered}
$$

\subsubsection{Level 3: Modality fusion and temporal filtering}

The so called hybrid positioning techniques are based on a combination of different methods introduced in Section 2.1.2 aiming to improve reliability, accuracy, and wireless resource consumption, among other performance characteristics.

Using measurements of different modality (kind) is not a problem and is covered in the same nonlinear set of equation framework as (2.1). The only difference is that other sensor information can be included. The inertial sensor unit in smartphones is today used to compute various motion related parameters. These can be used on the device for positioning, but also transmitted to the network. For 
instance, inertial sensor measurements can be combined with the global positioning system (GPS) for classification of the user's motion modes, see [70]. Fusion of TOF and TDOA measurements is another example of this type that is further discussed in later chapters.

The key idea with filtering is to include temporal correlation in a dynamic model, so that a prediction of the next position can be computed in a state-space model (SSM) framework. The unknown, unobserved states $x$ of the system in a SSM framework are inferred from the measurement function $\mathbf{h}(\cdot)$ and evolved in time using the transition function $\mathbf{f}(\cdot)$. Although for the linear class of SSM in white Gaussian noise a closed-form solution exists, nonlinear SSM require approximative approaches to compute the recursions. Further discussions on Bayesian filtering and corresponding solutions are provided in Section 3.2.

\subsection{Practical considerations}

This section continues the brief overview of radio network measurements given in Section 2.1.1, and provides a practical survey similar to [55], extended with recent measurements and standards. Lower layer techniques for providing these measurements are not addressed, and instead we refer to $[36,134]$ for 2nd generation $(2 \mathrm{G}),[22,135]$ for 3 rd generation $(3 \mathrm{G})$ and $[33,73]$ for 4 th generation $(4 \mathrm{G})$ cellular systems.

\subsubsection{Received signal strength}

In the RSS measurement (2.7a), in addition to the measurement noise $e_{t}^{(i), \text { RSS }}$, one might also consider the diffraction factor. This way, (2.7a) can be re-written as

$$
y_{t}^{(i), \text { RSS }}=P_{0}^{(i)}+10 \eta \log \left(\frac{\left\|\boldsymbol{\theta}_{t}-\boldsymbol{\ell}_{t}^{(i)}\right\|}{d_{0}}\right)+e_{t}^{(i), \mathrm{RSS}}+d_{t}^{(i), \mathrm{RSS}},
$$

where $d_{t}^{(i), \text { RSS }}$ is the diffraction. Propagation also features diffraction effects which resembles shadow fading that is a lowpass spatial process. A number of methods exists to deal with the diffraction error. One way is to lump them together with the measurement error, see [133] for more details. Another approach is to capture these variations in a model/database which essentially forms the fingerprinting method. A third way is to assume that the shadow fading is only present in the intermediate to far field from the antenna, but not in the near field. This way, in the near field, the only source of error is the measurement noise.

\subsubsection{TOA and TDOA}

Both TOF and TDOA are based on TOA measurements at the UE as well as the BS. TOA is estimated by cross-correlating the received signal with a replica of the transmitted signal waveform. TOA is used to estimate TOF by combining TOA estimated at BS and UE, while TDOA is estimated using TOA associated to two 
different BSs. [53] provides a novel method for round trip time calculations in LTE systems using the uplink timing alignment mechanism.

The performance analysis performed by [129], indicates different levels of accuracy based on the pilots used, as well as the bandwidth of the LTE system. Let $\sigma_{\mathrm{LB}}^{\mathrm{TOA}}$ denote the lower bound (LB) on achievable TOA estimation with $68 \%$ confidence interval when the pilot signal is received at UE with signal to noise ratio $(\mathrm{SNR})=-13 \mathrm{~dB}$. For an ideal additive white Gaussian noise (AWGN) channel, for a $20 \mathrm{MHz}$ LTE system using PRS, the lower bound on TOA standard deviation is $\sigma_{\mathrm{LB}}^{\mathrm{TOA}}=2.4 \mathrm{~ns}$. Assuming the signal is transmitted at $3 \times 10^{8} \mathrm{~m} / \mathrm{s}$, this translates to about $0.7 \mathrm{~m}$. Using the same pilot signal but reducing the system bandwidth to $1.4 \mathrm{MHz}$, the accuracy degrades to $\sigma_{\mathrm{LB}}^{\mathrm{TOA}}=66 \mathrm{~ns}$ or about $20 \mathrm{~m}$.

Assuming that two independent TOA measurements, with $\sigma_{\mathrm{LB}}^{1, \mathrm{TOA}}$ and $\sigma_{\mathrm{LB}}^{2, \mathrm{TOA}}$, are used to estimate the TDOA, the lower bound on the achievable accuracy is given by $\sigma_{\mathrm{LB}}^{\mathrm{TDOA}}=\sqrt{\left(\sigma_{\mathrm{LB}}^{1, \mathrm{TOA}}\right)^{2}+\left(\sigma_{\mathrm{LB}}^{2, \mathrm{TOA}}\right)^{2}}$. For the $20 \mathrm{MHz}$ and $1.4 \mathrm{MHz} \mathrm{LTE}$ systems, with the same setup as above, the accuracy levels are $1 \mathrm{~m}$ and $22 \mathrm{~m}$, respectively, see [129] for more details.

The emergency call positioning requirements by the FCC in the United States have been refined several times, initially with requirements on network-based positioning, and subsequently with tighter requirements on mobile-assisted positioning $[59,135]$. Recently, FCC has yet again refined the requirements to give particular attention to requirements for positioning of indoor devices [1].

These requirements are presented as a roadmap with stricter requirements over time, and considering all mobiles, both outdoors and indoors. The requirement is a horizontal accuracy corresponding to a dispatchable address or within a radius of $50 \mathrm{~m}$ for $40 \%$ of all wireless 911 calls within two years, gradually tightened to $80 \%$ of the wireless 911 calls within six years. The highest achievable accuracies for TOA and TDOA estimations are thus currently well in-line with the requirements of FCC even for the lowest bandwidth $(1.4 \mathrm{MHz})$ of LTE systems.

\subsubsection{Barometric pressure}

All indoor navigation systems, require a reliable source of vertical measurement (along the $z$-axis) in multi-story environments to operate with an acceptable level of accuracy. This information can be obtained for example from GPS-based elevation estimation techniques. However, lack of accuracy and reliability on top of limited availability to outdoor environments motivates more reliable source of information. One complementary sensor that solves the tricky vertical position problems is barometric pressure sensors that are based on barometric formula stating that atmospheric pressure decreases with increasing altitude.

Given a reference point at which the height above the see level $\ell_{z_{t}}^{(i)}$, standard air temperature $T_{r}$, and air pressure $p_{r}$ are known, see Figure $2.3, \theta_{z_{t}}$ can be found by

$$
\theta_{z_{t}}=p_{r}+\frac{T_{r}}{L}\left({\frac{p_{\theta}}{p_{r}}}^{-\tilde{c}}-1\right),
$$


where $\tilde{c}$ is the constant in barometric formula, $L$ is temperature lapse rate, and $p_{\theta}$ is the known air pressure at location of the UE. Generic measurement of the altitude of UE relative to the reference point is thus given by

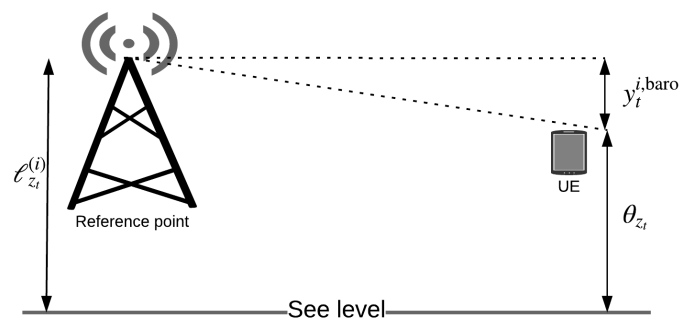

Figure 2.3: The measured elevation of the UE using known pressure at a reference point.

$$
y_{t}^{i, \text { baro }}=\left\|\theta_{z, t}-\ell_{z, t}^{(i)}\right\|+e_{t}^{i, \text { baro }} .
$$

An example of a possible use of a barometer in vertically oriented activities is presented in [94]. Three types of reference points exist:

- Meteorological stations for weather forecast already deployed by the national meteorological agencies. These stations have coarse spatial density on the amplitude of tens of kilometers and low update frequency of almost once an hour.

- The elevation of a person with a smartphone in outdoor environment taken from Digital Elevation Model (DEM)-map based on his current location is called a "DEM reference".

- The third reference point is based on an ad-hoc fashion of smartphones within the system.

For the case a reference pressure is unavailable, [84] presents a framework that does not depend on any special infrastructure and provides accurate elevation measurements using only smartphones. The final accuracy obtained by applying the system presented in [84] is less than $5 \mathrm{~m}$ in $90 \%$ of the cases and less than $3 \mathrm{~m}$ in $75 \%$ of times.

\subsection{Trends}

So far, we described the area of positioning in radio networks followed by practical consideration. However, there are some important trends that are expected to further improve the achievable accuracy. 


\subsubsection{New and better information}

\section{New timing measurements}

The timing measurement protocol was first introduced in the institute of electrical and electronics engineers (IEEE) $802.11 \mathrm{v}$ standard as an optional management for stations. Those stations who do not support this procedure, shall ignore a received timing measurement frame. Reference [44] presents the workflow of various wireless network management procedures of the IEEE $802.11 \mathrm{v}$ standard including timing measurements. Initiation of or stopping an ongoing procedure takes place by a "Request frame" sent by the receiving station. The value of the trigger field dictates if it is an initiative frame or a stop one.

Indoor environments, however, require more sophisticated measurement procedures to deal with practical challenges. For instance, while GNSS systems in outdoor environments are equipped with atomic clocks providing precise synchronization between all satellites, WiFi access points are not necessarily synchronized. The synchronization issue is compensated by measuring round-trip delays [76]. A yet newer protocol, fine timing measurement (FTM) [44], enables indoor TOF positioning. Measurements in the FTM protocol can be performed for different bandwidths. Additionally, the number of measurement frames is configurable and can take a value between 1 and 32. Figure 2.4 illustrates a generic implementation of FTM initiated with FTM request.

\section{Massive MIMO}

Classic array processing with multiple input multiple output (MIMO) antennas as surveyed in [75], enables accurate direction of arrival estimation. Massive MIMO, where the number of antenna elements is on order of magnitude larger than the number of communication links they serve, scales very favorably. This and many other advantages are described in [105].

In addition to the research on communications perspectives, as shown in [51], massive MIMO is also an enabler for accurate localization. Authors in [108] studied multiple users localization using fingerprinting solution by means of massive MIMO. Using the concept of direct localization, first introduced in [123], authors in [48] studied direct localization for massive MIMO.

\section{Ad-hoc networks}

Localization services that are applicable to these networks must meet different demands such as low power consumption, availability, and reliability. That is why some existing services such as GPS cannot be employed on wireless ad-hoc networks. To address this issue, one alternative is to use short-range single-hop localization systems. However, there are cases in which reference nodes are not in the range of unknown ones. Then, multi-hop techniques must be taken into account. In these scenarios, beacon positions are broadcasted over multiple hops. This allows estimation of the distance to beacon nodes by calculating hop sizes and number of hops. 


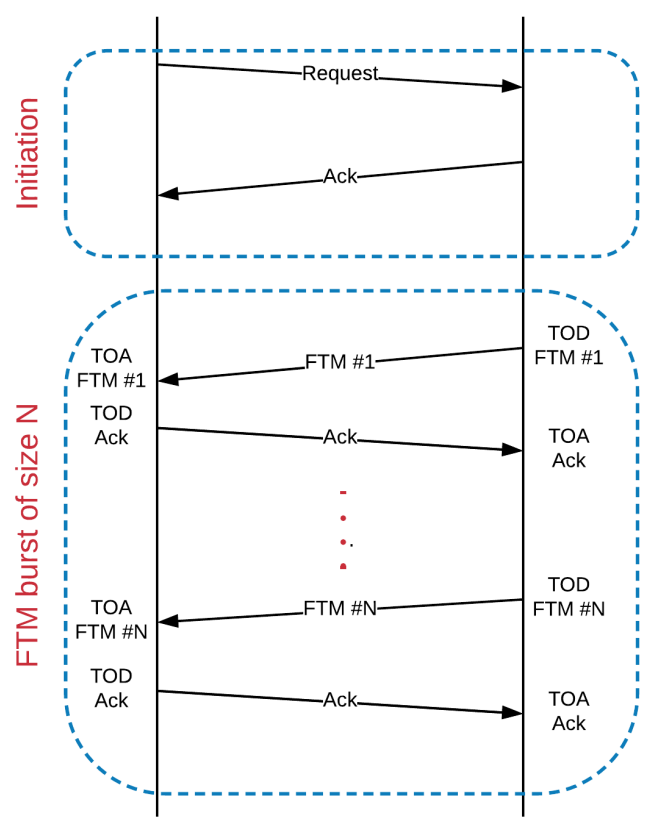

Figure 2.4: Fine timing measurement protocol with FTM burst size $N$ where $1 \leq N \leq 32$. Time of departure (TOD) and TOA are marked in the figure.

An ad-hoc positioning system based on AOA measurements is reported in [96]. Authors in [127] use a distance vector hop algorithm for wireless sensor networks based on the received signal strength indicator for positioning. Experimental results on ad-hoc networks that are self-organized by means of flying robots are studied in [104]. An extensive survey on position-based routing in vehicular adhoc networks is performed in [65].

\subsubsection{New infrastructure}

The infrastructure contains different entities that each of them can affect the measurement resolution drastically. All the devices at the lowest layer are connected to their upper layer devices via a short-range technology such as Bluetooth, ZigBee, etc. In the meantime, devices in the middle layer could vary from a simple UE acting as a gateway to a machine type communication (MTC) device [18]. Different types of access of the middle devices could be an IP-connectivity to another gateway, cellular access to the access point (AP) or even an intra-connection to another device of the same layer via a short range technology. 


\section{BLE beacons}

Bluetooth low energy (BLE) beacons can be low cost tiny computers equipped with Bluetooth radios. More complex hand-held devices such as smartphones can also provide the same functionality. The general idea is that these devices emit short-range signals that can be decoded by another BLE-enabled device. The distance to the receiving beacon can then be estimated. The possibility of identification of multiple beacons simultaneously in parallel with relative distance calculations of each beacon, location awareness of the device becomes possible.

\section{IoT}

Internet of things (IОT) can be seen as a great potential in many lines of research and development. However, massive signaling traffic produced by numerous objects that update their locations, causes new challenges that need to be addressed. Thus, there is a need for appropriate solutions that provide accurate location information while keeping the signaling level low.

\section{M2M}

Machine to machine (M2M) networks contain a number of devices such as radiofrequency identification, sensors, tags, etc. This type of network is employed in different location-based applications ranging from health monitoring to battlefield surveillance. M2M communication networks are self-configurable with the feature of being accessed remotely. The efficiency of approaches for location estimation of M2M network devices can be defined by scalability, whether or not they depend on GPS systems, range-based or range free property, and error handling capabilities.

\section{Fifth generation $(5 \mathrm{G})$}

Positioning is one of the most important design specifications for next generation 5G systems. Particularly, the millimeter wave technology operating in carrier frequencies beyond $30 \mathrm{GHz}$ band [11] has specific properties that make it of great interest for radio-based positioning [118]. The millimeter wave technology allows for packing massive arrays into a small area. For example, authors in [68] studied the problem of realizing millimeter-wave massive arrays with dimensions of a tablet.

The possibility of integrating a massive array in small areas, enabled by millimeter wave technology in 5G systems, motivated authors in [52], to study the concept of personal mobile radar operating at millimeter-waves. Positioning for vehicular networks using millimeter wave technology in 5G systems is studied in [126]. More recently, by taking advantage of large MIMO and millimeter-wave technologies, authors in [110] study the problem of positioning and orientation estimation using only one BS. 


\section{Narrowband loT}

Although GNSS solutions are capable of determining the position of an object with a few meters accuracy in outdoor environments, the robustness of GNSSbased methods is always restricted by the availability of GNSS signals. Indoor environments and dense urban areas are examples where these solutions fail.

As a response, 3GPP LTE standard features positioning support since 3GPP Release 9. The subsequent releases, as explained in [9], further extended capabilities of positioning by introducing specific signaling infrastructures. For more information on positioning in LTE systems see $[25,37,67]$.

The immense number of use cases inspired by IOT, however, motivated 3GPP to introduce Release 14, Narrowband IOT (NBIOT). Wearable technologies, asset tracking, environmental monitoring are examples of 'things' addressed by IOT. Low power consumption and the possibility to communicate in the most challenging locations, in terms of coverage, are among shared requirements in all these scenarios.

NBIOT aims to offer deployment flexibility allowing an operator to allocate a small portion of its current available spectrum to NBIOT. Co-existence performance with legacy GSM, general packet radio service (GPRS) and LTE technologies are primary design criterion for NBIOT. As reported in [122], NBIOT requires a minimum of $180 \mathrm{kHz}$ system bandwidth for both downlink and uplink. A GSM operator can replace one GSM carrier $(200 \mathrm{kHz})$ with NBIOT. An LTE operator can deploy NBIOT inside an LTE carrier by allocating one of the physical resource blocks (PRB) of $180 \mathrm{kHz}$ to NBIOT. 


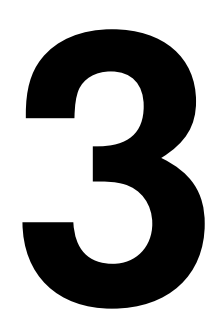

\section{Theoretical Background}

Localization in timing-based systems, in general, is based on the known position of the involved BSs. For instance, in TOF and TOA positioning systems, lateration is applied that uses the absolute distance between UE and the involved BSs. Using pairwise differences of the absolute distances to each BS gives the TDOA measurement, commonly referred to as hyperbolic localization. The measurements can be translated into hyperbolas with focal points located at the BSs on which the UE can be. Without any further processing, in all the three measurements, at least three BSs are required in $2 \mathrm{D}$ scenarios to locate the UE.

Combining measurements of different types, not limited to those obtained from radio networks, leads to hybrid approaches that can improve the accuracy and deal with possible ambiguities. For instance, fusing the information obtained from GNSS signals with inertial sensor measurements, it is possible to take advantage of the fast sampling-rate of inertial sensor observations and correct the drift using accurate GNSS position fixes [69].

Basic position estimators, introduced in Section 2.1.2, are based on the least squares principle and valid only for linear models. However, for hybrid methods with nonlinear models more complex algorithms are required to be able to fuse information obtained from different sources. Additionally, measurement noises in e.g. TDOA have spatial correlations resulting in symmetric but non-diagonal covariance matrices. In the rest of this section, the problem of static location estimation of the UE is studied. Then, modality fusion and temporal filtering introduced in Section 2.1.3 is discussed in more details for both linear and nonlinear models.

Let $h(\boldsymbol{\theta}, \boldsymbol{\ell})$ denote any type of the standard measurement models defined in Section 2.1.1 where type of measurements are discarded for sake of notation simplicity. The set of available measurements stacked in a vector is then given by $y=\boldsymbol{h}(\boldsymbol{\theta}, \boldsymbol{\ell})+\boldsymbol{e}$. 


\subsection{Cost function methods}

In the static case, there is no assumption of temporal correlation between consecutive positions, so the position vector is a sequence of uncorrelated parameters estimated in a snapshot manner.

\section{Problem formulation}

The UE can be localized by finding the position that minimizes a specific cost function $\mathcal{J}(\theta)$ that in general is presented by

$$
\hat{\theta}=\underset{\theta}{\arg \min } \mathcal{J}(\theta),
$$

where $\mathcal{J}(\boldsymbol{\theta})=\|\mathbf{y}-\boldsymbol{h}(\boldsymbol{\theta}, \boldsymbol{\ell})\|$ is one possibility. Different types of cost functions can be defined to estimate $\theta$ from known measurements $y$ corrupted by the stochastic unknown error $\boldsymbol{e}$. For instance, if the probability distribution of the error, $p_{e}(\boldsymbol{e})$ is known, the maximum likelihood estimator (MLE) corresponding to the cost function

$$
\mathcal{J}^{M L}(\boldsymbol{\theta})=\log p_{e}(\boldsymbol{y}-\boldsymbol{h}(\boldsymbol{\theta})),
$$

can be used. The MLE is known to be asymptotically efficient [72]. In the special case where measurement noises are independent, identically Gaussian distributed, $p_{e}(\boldsymbol{e}) \sim \mathcal{N}\left(\boldsymbol{e} ; \mathbf{0}, \sigma_{e}^{2} \boldsymbol{I}\right)$, the MLE turns into a nonlinear least squares (NLS) problem

$$
\mathcal{J}^{N L S}(\boldsymbol{\theta})=(\boldsymbol{y}-\boldsymbol{h}(\boldsymbol{\theta}))^{T}(\boldsymbol{y}-\boldsymbol{h}(\boldsymbol{\theta})) .
$$

In case of spatial correlations in measurement noise terms $p_{e}(\boldsymbol{e}) \sim \mathcal{N}(\boldsymbol{e} ; \mathbf{0}, \boldsymbol{R}(\boldsymbol{\theta}))$, the weighted version of NLS, WNLS, should be used instead

$$
\mathcal{J}^{W N L S}(\boldsymbol{\theta})=(\boldsymbol{y}-\boldsymbol{h}(\boldsymbol{\theta}))^{T} \boldsymbol{R}^{-1}(\boldsymbol{\theta})(\boldsymbol{y}-\boldsymbol{h}(\boldsymbol{\theta})) .
$$

\section{Optimization methods}

Irrespective of which optimization criteria in (3.2) is considered, in general, there is no closed form solution to (3.1) and numerical optimization methods are required [54]. Initialized at $\hat{\theta}_{0}$, these methods produce a sequence of iterative updates $\left\{\hat{\theta}_{k}\right\}_{k=0}^{\infty}$. In each iteration $k$, the algorithm computes the cost function at $\hat{\theta}_{k}$ to decide how to move to the next iterate such that $\mathcal{J}\left(\hat{\theta}_{k}\right)<\mathcal{J}\left(\hat{\theta}_{k-1}\right)$. The iterations terminate either at a given threshold or when no more progress is made.

Two fundamental strategies for iterative updates of the unknown parameter are line search and trust region approaches. In this thesis we consider the line search method in which the iteration is defined by

$$
\hat{\theta}_{k}=\hat{\theta}_{k-1}+\alpha_{k} \boldsymbol{p}_{k}
$$

where $p_{k}$ is the search direction and $\alpha_{k}$ is the step length that defines how far to move along the direction $\boldsymbol{p}_{k}$. Given an appropriate step length $\alpha_{k}$ at each 
iteration, any direction with an angle of less than $\pi / 2$ radian with $-\nabla_{\theta} \mathcal{J}(\hat{\theta})_{k}$ is called a descent direction. Mainly, all line search algorithms consider a descent direction $\boldsymbol{p}_{k}$ that satisfies $\boldsymbol{p}_{k}^{T} \nabla_{\theta} \mathcal{J}\left(\hat{\boldsymbol{\theta}}_{k}\right)<0$. This guarantees the reduction in the cost function if we move along this direction [97]. The general form of the search direction in line search methods is given by

$$
\boldsymbol{p}_{k}=-\Upsilon_{k}^{-1} \nabla_{\boldsymbol{\theta}} \mathcal{J}\left(\hat{\boldsymbol{\theta}}_{k}\right),
$$

where $\Upsilon_{k}$ is a symmetric and non-singular matrix. Two local search algorithms that are widely used in positioning applications are steepest descent and GaussNewton [34] as defined in the rest of this section.

Define $\boldsymbol{H}(\boldsymbol{\theta})=\nabla_{\boldsymbol{\theta}} \boldsymbol{h}(\boldsymbol{\theta})$ for the least squares cost functions and $\boldsymbol{H}(\boldsymbol{\theta})=\nabla_{\boldsymbol{\theta}} \log p_{e}(\boldsymbol{y}-\boldsymbol{h}(\boldsymbol{\theta}))$ for MLE. It must be noted that in what follows, $\mathbf{R}$ should be set to an identity matrix of appropriate size if either of cost functions $(3.2 \mathrm{a})$ or $(3.2 \mathrm{~b})$ are to be optimized.

\section{Steepest descent}

The most obvious choice of the search direction is the one used in steepest descent where $\boldsymbol{\Upsilon}_{k}$ is the identity matrix, hence $\boldsymbol{p}_{k}=-\nabla_{\boldsymbol{\theta}} \mathcal{J}\left(\hat{\boldsymbol{\theta}_{k}}\right)$. The steepest descent iterations are given by

$$
\hat{\boldsymbol{\theta}}_{k}=\hat{\boldsymbol{\theta}}_{k-1}+\alpha_{k} \boldsymbol{H}^{T}\left(\hat{\boldsymbol{\theta}}_{k-1}\right) \boldsymbol{R}^{-1}\left(y-\boldsymbol{h}\left(\hat{\boldsymbol{\theta}}_{k-1}\right)\right) .
$$

As discussed in [97], the steepest descent algorithm is globally convergent but might be very slow in practice.

\section{Gauss-Newton}

Newton's method uses information of the exact Hessian matrix to find the search direction. This guarantees a descent direction given that the exact Hessian matrix is positive definite. Otherwise, a search direction might not even exist and should be approximated using Hessian modification methods that might result in a direction that is not descent [97]. Gauss-Newton is an approximated version of the Newton's method that uses an approximate Hessian (as in Levenberg-Marquardt method), $\nabla_{\boldsymbol{\theta}}^{2} \mathcal{J}\left(\hat{\boldsymbol{\theta}}_{k}\right) \approx 2 \boldsymbol{J}\left(\hat{\boldsymbol{\theta}}_{k}\right)^{T} \boldsymbol{J}\left(\hat{\boldsymbol{\theta}}_{k}\right)$ and exact gradient [34], where $\boldsymbol{J}(\hat{\boldsymbol{\theta}})$ is the Jacobian matrix. The Gauss-Newton iterations are given by

$$
\hat{\boldsymbol{\theta}}_{k}=\hat{\boldsymbol{\theta}}_{k-1}+\alpha_{k}\left(\boldsymbol{H}^{T}\left(\hat{\boldsymbol{\theta}}_{k-1}\right) \boldsymbol{R}^{-1} \boldsymbol{H}\left(\hat{\boldsymbol{\theta}}_{k-1}\right)\right)^{-1} \boldsymbol{H}^{T}\left(\hat{\boldsymbol{\theta}}_{k-1}\right) \boldsymbol{R}^{-1}\left(y-\boldsymbol{h}\left(\hat{\boldsymbol{\theta}}_{k-1}\right)\right)^{T} .
$$

Note that in the exact Newton's method, $\Upsilon_{k}$ is the exact Hessian $\nabla_{\theta}^{2} \mathcal{J}\left(\hat{\boldsymbol{\theta}}_{k}\right)$ giving the search direction $\boldsymbol{p}_{k}=-\left(\nabla_{\boldsymbol{\theta}}^{2} \mathcal{J}\left(\hat{\boldsymbol{\theta}}_{k}\right)\right)^{-1} \nabla_{\boldsymbol{\theta}} \mathcal{J}\left(\hat{\boldsymbol{\theta}}_{k}\right)$.

\section{Gauss-Newton with modified step lengths}

Possibility of convergence to a local optima is a well-known problem with all line search methods that might occur in case of poor initialization of the iterations. 
Rate of convergence is yet another important factor in all these optimization algorithms that need to be addressed while designing them. These two criteria can sometimes conflict with each other, hence extra care needs to be taken. Section 4.2 employs the modified Gauss-Newton method with varying step lengths as in [77] to improve both the convergence rate and global convergence.

Additive updates in each iteration, $\alpha_{k}$ are damped by using $\alpha_{k} \in(0,1]$

$$
\begin{aligned}
\mathcal{J}_{k}\left(\alpha_{k}\right) & :=\mathcal{J}\left(\hat{\boldsymbol{\theta}}_{k-1}+\alpha_{k} \boldsymbol{p}_{k}\right) \\
M & :=\left\{\alpha_{k} \in\left\{1, \frac{1}{2}, \frac{1}{4}, \frac{1}{8}\right\}: \mathcal{J}_{k}\left(\alpha_{k}\right)<\mathcal{J}_{k}(0)\right\} \\
\alpha_{k} & =\left\{\begin{array}{cc}
\frac{1}{8} & M=\emptyset \\
\max (M) & \text { otherwise. }
\end{array}\right.
\end{aligned}
$$

\subsection{Bayesian filtering}

Bayesian filters are recursive algorithms used to infer the states of dynamic systems from noisy observations. In localization context, the state can be the UE's location while indirect observations of the state are given by e.g. timing measurements. In each recursion, the previous state estimate together with a priori known dynamics of the system and current observations are used to update the state estimate.

\section{Statistical system models}

The statistical dynamic model explains how the states evolve in time and is given by

$$
x_{t+1} \sim p\left(x_{t+1} \mid x_{t}\right)
$$

The statistical measurement models are used to relate the collected observations to the system states as

$$
y_{t} \sim p\left(y_{t} \mid x_{t}\right)
$$

Assuming that the states of the system, $\boldsymbol{x}_{t}$, follow a Markovian property

$$
\begin{aligned}
p\left(x_{t+1} \mid x_{1} \ldots x_{t}\right) & =p\left(x_{t+1} \mid x_{t}\right), \\
p\left(y_{t} \mid x_{1} \ldots x_{t}, y_{1} \ldots y_{t-1}\right) & =p\left(y_{t} \mid x_{t}, y_{1} \ldots y_{t-1}\right) .
\end{aligned}
$$

Furthermore, noting that given $x_{t}, y_{t}$ is conditionally independent of $y_{1}, \ldots, y_{t-1}$

$$
p\left(y_{t} \mid \boldsymbol{x}_{t}, y_{1} \ldots y_{t-1}\right)=p\left(y_{t} \mid \boldsymbol{x}_{t}\right) \text {. }
$$

The posterior densities are estimated recursively in the Bayesian framework, initialized at the prior distribution of states $p\left(x_{0}\right)$, see [63] and [107]. 


\section{Recursive state estimate}

Given the set of measurements $\boldsymbol{Y}_{1: t}=\left[y_{1}, \ldots, y_{t}\right]$, the state estimation problem is the problem of finding the predictive $p\left(\boldsymbol{x}_{t+1} \mid \boldsymbol{Y}_{1: t}\right)$ and filtering $p\left(\boldsymbol{x}_{t} \mid \boldsymbol{Y}_{1: t}\right)$ posterior distributions. The one-step ahead predictive distribution for a given dynamic model is given by

$$
p\left(\boldsymbol{x}_{t+1} \mid \boldsymbol{Y}_{1: t}\right)=\int p\left(\boldsymbol{x}_{t+1} \mid \boldsymbol{x}_{t}\right) p\left(\boldsymbol{x}_{t} \mid \boldsymbol{Y}_{1: t}\right) \mathrm{d} \boldsymbol{x}_{t}
$$

The filtering distribution then further includes the information obtained from measurements $y_{t}$ resulting in

$$
p\left(\boldsymbol{x}_{t} \mid \boldsymbol{Y}_{1: t}\right)=\frac{p\left(y_{t} \mid \boldsymbol{x}_{t}\right) p\left(\boldsymbol{x}_{t+1} \mid \boldsymbol{Y}_{1: t}\right)}{p\left(y_{t} \mid \boldsymbol{Y}_{1: t-1}\right)},
$$

where the normalization constant in (3.11a) is defined as

$$
p\left(y_{t} \mid \boldsymbol{Y}_{1: t-1}\right)=\int p\left(y_{t} \mid \boldsymbol{x}_{t}\right) p\left(\boldsymbol{x}_{t} \mid \boldsymbol{Y}_{1: t-1}\right) \mathrm{d} \boldsymbol{x}_{t}
$$

In this thesis, we restrict our analysis to discrete-time filtering of sampled signals denoted by subscript $k$. The snapshot positions $\theta$ are then indexed by $k$, stacked in the states of the system dynamics $\boldsymbol{x}_{k}$. Bayesian filters can then be applied to derive the filtering distribution of the state of the system at time instance $k$ given all the measurements up to $k$.

Point estimates of the state vector at each time instance $k$, if desired, can then be computed from the marginal posterior distribution $p\left(\boldsymbol{x}_{k} \mid \boldsymbol{Y}_{1: k}\right)$. For example, choosing the point estimate that gives the minimum mean squared error (MMSE) gives the conditional mean of the state $\boldsymbol{x}_{k}$ given the measurements $\boldsymbol{Y}_{1: k}$. MMSE is in fact the optimal solution to the optimization of the expected loss function $\mathbb{E}\left\{\mathcal{J}(\boldsymbol{x}) \mid \boldsymbol{Y}_{1: k}\right\}$ when a weighted quadratic loss function is to be optimized. Maximum a posteriory (MAP) is another alternative that gives the most probable value of the sates as the point estimate. In the special case of Gaussian posterior distribution, the two solutions coincide,

$$
\begin{aligned}
\hat{\boldsymbol{x}}_{k \mid k}^{\mathrm{MMSE}} & =\mathbb{E}_{\boldsymbol{x}_{k}}\left\{\boldsymbol{x}_{k} \mid \boldsymbol{Y}_{1: k}\right\}, \\
\hat{\boldsymbol{x}}_{k \mid k}^{\mathrm{MAP}} & =\underset{\boldsymbol{x}_{k}}{\arg \max } p\left(\boldsymbol{x}_{k} \mid \boldsymbol{Y}_{1: k}\right) .
\end{aligned}
$$

For the general statistical model (3.8), closed-form solutions to the recursions (3.10) and (3.11a) might not always exist. In the rest of this section we first consider the special case in which (3.8) corresponds to a linear Gaussian SSM for which analytical solutions exist. Then, one alternative for approximating nonlinear SSM is introduced. 


\section{Linear models; closed form solution}

In the special case when both the dynamic and the measurement model are linear, we obtain the class of linear SSM,

$$
\begin{aligned}
\boldsymbol{x}_{k+1} & =\boldsymbol{F}_{k} \boldsymbol{x}_{k}+\boldsymbol{\omega}_{k+1}, \\
y_{k} & =\boldsymbol{H}_{k} \boldsymbol{x}_{k}+\boldsymbol{e}_{k},
\end{aligned}
$$

where $\omega_{k} \sim p\left(\omega_{k}\right)=\mathcal{N}\left(\omega_{k} \mid \mu_{k}^{\omega}, Q_{k}\right) \in \mathcal{R}^{n_{\omega}}$ denote the disturbances entering the system as input and the vector $\boldsymbol{e}_{k} \sim p\left(\boldsymbol{e}_{k}\right)=\mathcal{N}\left(\boldsymbol{e}_{k} \mid \boldsymbol{\mu}_{k}^{e}, \boldsymbol{R}_{k}\right) \in \mathcal{R}^{n_{e}}$ is the measurement noise.

The well-known Kalman filter (KF) provides an optimal closed form solution to the estimation problem. Assuming zero-mean noise terms, $\boldsymbol{\mu}_{K}^{e}=\boldsymbol{\mu}_{K}^{\omega}=\mathbf{0}, \mathrm{Al}$ gorithm 1 presents KF steps starting the recursions from initial state $x_{0}$ with the prior uncertainty $\boldsymbol{P}_{0}$. See [54], and [107] for proof. The algorithm can be used to obtain parameters of the following distributions

- Predictive distribution:

$$
p\left(\boldsymbol{x}_{k+1} \mid \boldsymbol{Y}_{1: k}\right)=\mathcal{N}\left(\boldsymbol{x}_{k+1} \mid \boldsymbol{x}_{k+1 \mid k}, P_{k+1 \mid k}\right) .
$$

- Filtering distribution:

$$
p\left(\boldsymbol{x}_{k} \mid \boldsymbol{Y}_{1: k}\right)=\mathcal{N}\left(\boldsymbol{x}_{k} \mid \boldsymbol{x}_{k \mid k}, \boldsymbol{P}_{k \mid k}\right)
$$

\section{Nonlinear models; approximate solutions}

In many practical applications, as the ones introduced in Section 2.1.1, the measurement model is not linear. In cases when either one or both of the dynamic and measurement models are nonlinear, approximations are required. Assuming that the noise terms enter the system additively, the nonlinear SSM is given by

$$
\begin{aligned}
\boldsymbol{x}_{k+1} & =\boldsymbol{f}_{k}\left(\boldsymbol{x}_{k}\right)+\boldsymbol{\omega}_{k+1} \\
y_{k} & =\boldsymbol{h}_{k}\left(\boldsymbol{x}_{k}\right)+\boldsymbol{e}_{k} .
\end{aligned}
$$

The solution to the nonlinear filtering problem can be approximated in different ways. One general framework is to solve the problem by approximating nonGaussian densities, resulting from nonlinearities in the model, by Gaussian densities. See [125], [16], [17], and [15] for more details on generalized Gaussian filters applications.

Special cases of general Gaussian filters can also be derived using Taylor series approximations of nonlinear models as in the extended Kalman filter (EKF), see, [114] and [63]. 


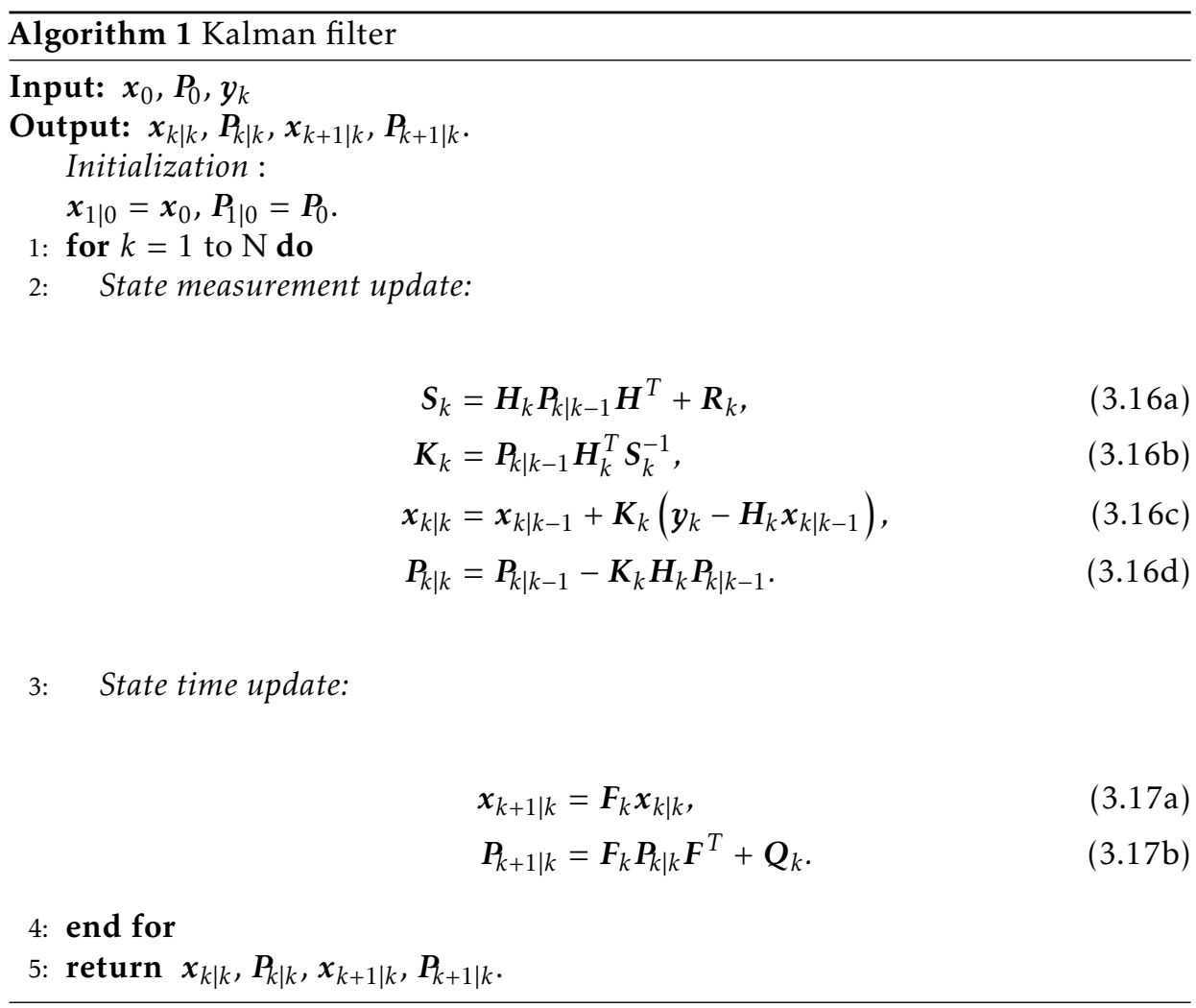

The EKF assumes that the posterior distribution is Gaussian $p\left(\boldsymbol{x}_{k} \mid \boldsymbol{Y}_{1: k}\right) \simeq$ $\mathcal{N}\left(\boldsymbol{x}_{k} \mid \overline{\boldsymbol{x}}_{k \mid k}, \overline{\boldsymbol{P}}_{k \mid k}\right)$ and approximates the first two moments. Algorithm 2 summarizes the steps of the EKF filtering method for the nonlinear SSM model (3.18) with additive noise.

The EKF estimated distributions, and their corresponding point estimates, are no longer optimal but the algorithm is simple and performs fairly well in many applications [66]. However in cases with severe nonlinearity, the algorithm might have poor performance as it is based on a local linear approximation. Performance improvements might be possible for example by employing information in the Hessian of the nonlinear models using a second order Taylor expansion. However, this further restricts the algorithm to models for which both first and second derivatives are available. In cases where the dynamic model is linear, and the measurement model is nonlinear the iterated EKF can provide performance improvements [19]. The idea with the iterated EKF is to better approximate the measurement model by iteratively repeating the measurement update phase (3.19) until a certain criteria is satisfied. 


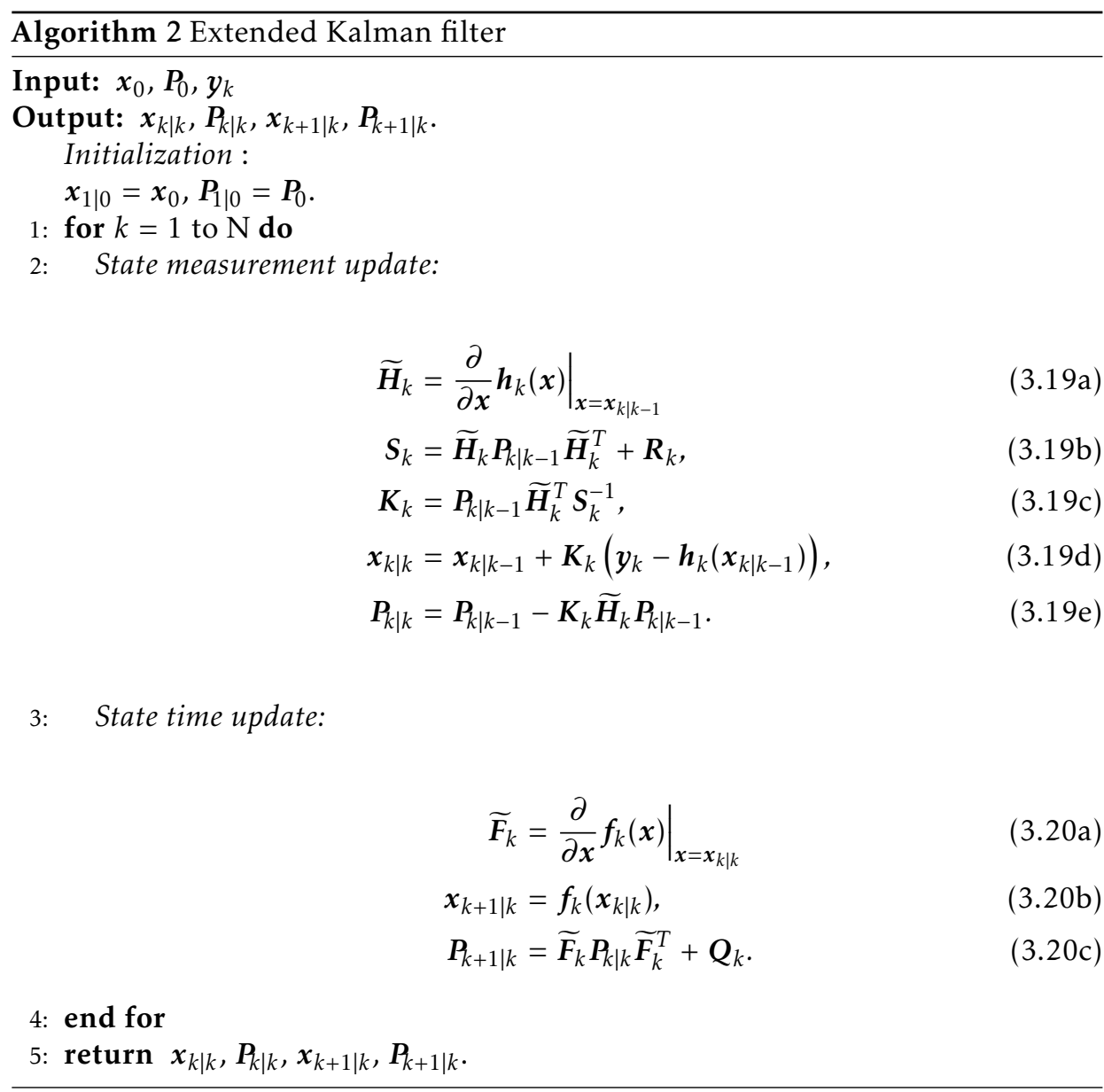

\section{Two examples of system dynamics}

The simplest system dynamics corresponds to the case where the UE is assumed to have nearly constant position manipulated by a zero-mean stochastic velocity known as random walk, nearly constant position model or constant position (CP) model [80]. In this case, the state vector contains only the position of the UE and in two-dimensional scenarios, this implies that $x_{k}=\left(\theta_{x, k}, \theta_{y, k}\right)^{T}$. Assuming that the disturbances $\omega_{k}$ in (3.14) are modeled as white Gaussian noise and enter the system additively,

$$
\begin{aligned}
& \boldsymbol{x}_{k+1}=\boldsymbol{F}^{(\mathrm{CP})} \boldsymbol{x}_{k}+\omega_{k+1}, \\
& \omega_{k+1} \sim \mathcal{N}\left(\mathbf{0}_{2}, Q^{(\mathrm{CP})}\right),
\end{aligned}
$$


with

$$
\boldsymbol{F}^{(\mathrm{CP})}=\boldsymbol{I}_{2}, \quad \boldsymbol{Q}^{(\mathrm{CP})}=\sigma_{\omega}^{2}\left(\begin{array}{ll}
T & 0 \\
0 & T
\end{array}\right)\left(\begin{array}{cc}
T & 0 \\
0 & T
\end{array}\right)^{T},
$$

where $\sigma_{\omega}$ is the noise standard deviation in $\mathrm{m} / \mathrm{s}$ and $T$ is the sampling interval.

In some applications, it is useful to further extend the state vector by the velocity of the UE. The assumption is that the velocity of the UE is fairly constant, and the acceleration is modeled as noise entering the dynamics. This is referred to as a nearly constant velocity model or constant velocity (CV) model [80]. The state vector in $2 \mathrm{D}$ then becomes $\boldsymbol{x}_{k}=\left(\theta_{x, k}, \theta_{y, k}, v_{x, k}, v_{y, k}\right)^{T}$ where $v_{x}$ and $v_{y}$ are the velocities in $x$ and $y$ directions, respectively. The dynamics of the system are then given by

$$
\begin{gathered}
\boldsymbol{x}_{k+1}=\boldsymbol{F} \boldsymbol{x}_{k}+\boldsymbol{\omega}_{k+1}, \\
\omega_{k+1} \sim \mathcal{N}\left(\mathbf{0}, \boldsymbol{Q}^{(\mathrm{CV})}\right),
\end{gathered}
$$

with

$$
\boldsymbol{F}^{(\mathrm{CV})}=\left(\begin{array}{cc}
\mathbf{I}_{2} & T \mathbf{I}_{2} \\
\mathbf{0} & \mathbf{I}_{2}
\end{array}\right), \quad \mathbf{Q}^{(\mathrm{CV})}=\sigma_{\omega}^{2}\left(\begin{array}{c}
\frac{T^{2}}{2} \mathbf{I}_{2} \\
T \mathbf{I}_{2}
\end{array}\right)\left(\begin{array}{c}
\frac{T^{2}}{2} \mathbf{I}_{2} \\
T \mathbf{I}_{2}
\end{array}\right)^{\mathrm{T}} .
$$

The process noise standard deviation for $\mathrm{CV}$ models is in $\mathrm{m} / \mathrm{s}^{2}$. For more information, see the survey article [80] that reports different types of motion models used in target tracking applications.

\section{Jump Markov models}

In cases where the system has multiple operational modes, the general statistical model (3.8) needs to be extended accordingly. For example, consider the simplified tracking problem based on the measurement model $\tilde{y}(t)=\tilde{x}^{2}(t)+\tilde{e}(t)$, where $\tilde{x}(t)$ is a slowly varying scalar process. We could in principle solve for $\tilde{x}(t)$ and get $\tilde{x}(t)= \pm \sqrt{\tilde{y}(t)-\tilde{e}(t)}$, and the core problem is which branch to select, the positive or negative one.

One alternative to handle multi-modality in the positioning problem is to derive the filtering distribution of the states of a jump Markov model (JMM). In general, JMM is a state-space model where both the motion and measurement models can depend on the mode of the system, $\delta_{t}$. The general class of JMMs without any assumptions on the system and noise models are defined as

$$
\begin{gathered}
\boldsymbol{x}_{k+1}=f\left(\boldsymbol{x}_{k}, \omega_{k}, \delta_{k}\right), \\
y_{k}=h\left(\boldsymbol{x}_{k}, \boldsymbol{e}_{k}, \delta_{k}\right),
\end{gathered}
$$

where the set of possible modes of the system is given by $\delta_{k} \in S$. In this thesis, the set of modes $S$ is assumed to contain integers. Additionally, the mode exhibits Markov property. Switching between the modes can either be deterministic or be 
given by an additional model $\Pi_{k}^{a, b}$ denoting the probability of transition from mode $a$ to mode $b$.

The filtering distribution of JMM can be computed in a filter bank framework. A filter bank can then be applied to ensure keeping track of all possible modes of the system at each time instant. Positioning using a bank of KFs is further investigated in Section 5.1.2 for linear JMM with additive noise terms. 


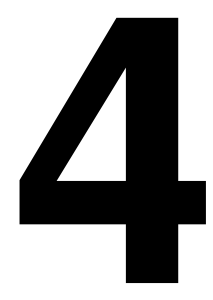

\section{OTDOA Positioning in NBIOT Systems}

Cellular communication networks, as discussed in [111], play an important role in IOT applications. Contrary to the broadband services in which high data rates are required, in most cases, lower data rates are acceptable for IOT applications [83] but with tighter requirements on better coverage, lower power consumption, and cheaper devices [3]. Thus, 3GPP developed two machine type communication (MTC) technologies, LTE MTC (LTE-M) and NBIOT, introduced in its Release 13 for low power wide area IOT connectivity. Limited positioning support for both LTE$M$ and NBIOT in Release 13, motivated 3GPP for improvements in Release 14 [83].

LTE-M is based on LTE and operates on a minimum system bandwidth of 1.4 $\mathrm{MHz}$ but with additional features resulting in better support for IOT services [103]. NBIOT, on the other hand, is a new radio access technology that requires $180 \mathrm{KHz}$ system bandwidth allowing for more deployment flexibility [122].

This chapter investigates OTDOA positioning in NBIOT systems where at least three base stations are detected by the UE to form timing measurements. We first motivate the research by giving a number of use cases of IOT positioning in Section 4.1 followed by the formulation of the OTDOA positioning in NBIOT systems given in Section 4.2. Section 4.3 presents different scenarios and a simulated NBIOT network followed by performance evaluations discussed in Section 4.4.

\subsection{Use cases of IOT positioning}

The intensive expansion of IOT could be seen as a result of rapid growth in use cases where machine-type communications are needed. The existing connectivity solutions fail to satisfy the three main requirements of wide coverage area, low cost device design, and low power consumption, simultaneously. Hence, the key features and capabilities that are to be addressed and optimized in NBIOT are, UE complexity reduction, long battery life and deeper coverage to reach challenging 
locations such as deep inside buildings. The new technologies also can leverage existing LTE infrastructure and spectrum, coexisting with today's mobile broadband services.

Tracking, logistics and wearables are all exemplary IOT use cases in which position awareness is required. For example, in indoor localization applications where GNSS solutions fail, NBIOT modules are a promising alternative. Tracking with low sampling rate is another use case in which relying on a NBIOT module as a low-cost alternative to a GSM tracker, is beneficial. GNSS based solutions might not always be the best alternative due to lost connectivity as well as high power consumption. Base stations might also be far away from the location of the user which requires the UE's transmitter to operate at high power.

Depending on the use case, the required positioning accuracy might vary ranging from a few meters up to hundreds of meters. The authors in [83] report that the benchmark target for LTE-M and NBIOT has been $50 \mathrm{~m}$ horizontal accuracy.

\subsection{OTDOA positioning in NBIOT systems}

To enhance TOA measurement precision, resulting in higher OTDOA positioning accuracy, LTE introduced PRS. NBIOT systems are also equipped with a specific PRS, narrowband positioning reference signal (NPRS), supporting downlink OTDOA based positioning. Specifically, for in-band deployment, PRS symbols are reused also in the NPRS, which typically is extended with a large number of repetitions to allow accumulation at the device for detectability. The signal to interference and noise ratio (SINR) threshold requirement for reference and neighboring cells of NBIOT devices compared to LTE systems is also improved. This results in better positioning support with OTDOA techniques in NBIOT devices compared to the legacy LTE as finding the minimum three cells for trilateration is more probable.

In the rest of this section, we present different aspects of IOT positioning within LTE standardization. NPRS transmission schemes compensating lower bandwidth of NBIOT systems together with the principle of reference signal time difference (RSTD) estimation are briefly explained.

\subsubsection{IOT positioning in LTE standardization}

Devices involved in IOT use cases require low-power consumption while demanding a certain level of positioning accuracy. They might be located indoors where GPS signals are not detectable or even might not support GPS or any other GNSS. Thus, the primary objective of NBIOT is to provide a radio access technology that allows for low device complexity, with low power consumption while still providing an adequate throughput for the connected devices.

\section{NPRS transmission schemes}

The reference signals used for TOA estimation are transmitted at so-called positioning occasions [40]. In LTE systems, in each positioning occasion, $N_{\text {PRS }}$ consec- 
utive subframes are sent every $T_{\mathrm{PRS}}$ subframe. In legacy LTE, $N_{\mathrm{PRS}}$ can be 1,2 , 4 , or 6 , while $T_{\text {PRS }}$ can be $160,320,640$ or $1280 \mathrm{~ms}$, see [6]. Figure 4.1 shows an example of positioning occasion corresponding to a $20 \mathrm{MHz}$ LTE system with 100 Resource Blocks (RB). The bandwidth of each RB is $180 \mathrm{kHz}$ and the PRS, in this example, has $10 \mathrm{MHz}$ bandwidth. $\Delta_{\mathrm{PRS}}$ is the cell specific subframe offset which defines the starting subframe of the PRS transmission relative to the start of the system frame cycle.

For NBIOT, the carrier bandwidth is $180 \mathrm{kHz}$, which fills up one LTE RB. To address the requirements of IOT use cases, NBIOT systems must ensure coverage and good performance in challenging indoor environments. Thus, denser NPRS transmissions compared to the legacy LTE PRS configuration are needed to support aggregation of data for adequate coverage.

Denser NPRS transmission occasions is achieved by extending $N_{\text {NPRS }}$ to also include $10,20,40,80$ or 160 subframes. Figure 4.1 illustrates an example of NBIOT NPRS transmission. Narrower bandwidth, as shown in the figure, can be compensated by longer $N_{\text {NPRS }}$. Supporting $N_{\text {NPRS }}=160$ subframes, it is possible to configure a transmission schedule to use all NBIOT resources, enabling even a continuous NPRS.

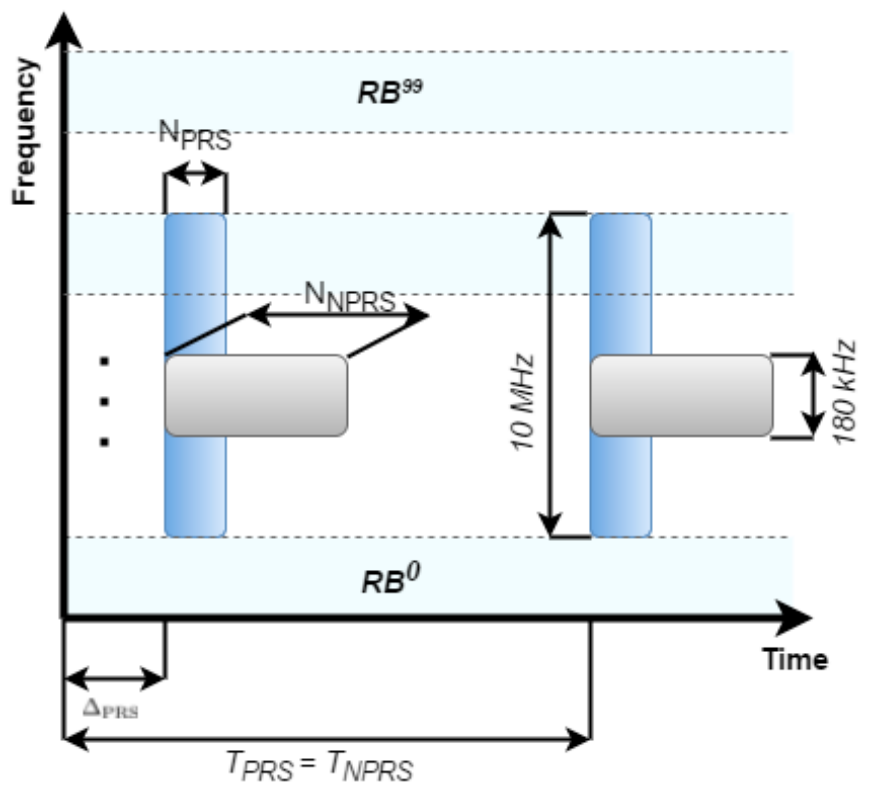

Figure 4.1: Example of the PRS and NPRS transmission schemes for a $20 \mathrm{MHz}$ LTE system with $10 \mathrm{MHz}$ PRS bandwidth and an in-band deployment of NBIOT system with $180 \mathrm{kHz}$ NPRS bandwidth. Maximum number of $N_{\text {PRS }}$ is 6 subframes. The narrower bandwidth of the NPRS is compensated by allowing $N_{\mathrm{NPRS}}$ to be increased up to 160 subframes [101, C2017 IEEE]. 
Table 4.1: Parameters of EPA and ETU tapped-delay LTE channel models.

\begin{tabular}{c|cc|cc} 
Tap & \multicolumn{2}{|c|}{ EPA channel } & \multicolumn{2}{c}{ ETU channel } \\
& $\begin{array}{c}\text { tap delay } \\
{[\mathrm{ns}]}\end{array}$ & $\begin{array}{c}\text { Relative power } \\
{[\mathrm{dB}]}\end{array}$ & $\begin{array}{c}\text { tap delay } \\
{[\mathrm{ns}]}\end{array}$ & $\begin{array}{c}\text { Relative power } \\
{[\mathrm{dB}]}\end{array}$ \\
\hline 1 & 0 & 0.0 & 0 & -1.0 \\
\hline 2 & 30 & -1.0 & 50 & -1.0 \\
\hline 3 & 70 & -2.0 & 120 & -1.0 \\
\hline 4 & 90 & -3.0 & 200 & 0.0 \\
\hline 5 & 110 & -8.0 & 230 & 0.0 \\
\hline 6 & 190 & -17.2 & 500 & 0.0 \\
\hline 7 & 410 & -20.8 & 1600 & -3.0 \\
\hline 8 & & & 2300 & -5.0 \\
\hline 9 & & & 5000 & -7.0 \\
\hline
\end{tabular}

\subsubsection{Channel model}

There are different channel models recommended for specific propagation environments [32] where they typically characterize multipath fading, path-loss attenuation, and shadow fading. For an extensive survey on wireless channel modeling see for example [93].

The tapped-delay models are based on ITU-R [119] and 3GPP [2] channel models where each tap identifies a multipath signal. Depending on the environment, 3GPP agreed on using pedestrian A from [119] for low delay spread and typical urban from [2] for high delay spread as stated in [8].

The evolution of LTE towards higher operating bandwidths, however, required revisions in the channel models [116]. An extension of the previous channel models were thus introduced and named EPA and ETU whose detailed parameters are described in [4] and [5]. These models can be also extended to multiple antenna scenarios, as described in [136] by considering spatial correlations as identified in [7]. The multipath profile corresponding to EPA and ETU is summarized in Table 4.1. Multipath in EPA and ETU is modeled as series of amplitude weighted delayed copies of the input signal.

\subsubsection{RSTD estimation}

The UE measurement for OTDOA positioning is the RSTD which is the relative time difference between the BS, or evolved node $\mathrm{b}(\mathrm{eNB}), j$ and the reference eNB $i$. RSTD is calculated as the smallest time difference between two subframe boundaries received from two different eNBs.

This thesis focuses on the impact of multipath fading propagation conditions on the RSTD estimations. This work considers this phenomena using a tappeddelay line channel model . Let $L$ be the number of multipath channels with com- 
plex valued gains $\alpha_{\ell}$, the tapped delay line model is then given by

$$
h(t)=\sum_{\ell=0}^{L-1} \alpha_{\ell} \delta\left(t-\tau_{\ell}\right),
$$

where $\tau_{\ell}$ indicates the time delay of the $\ell^{\text {th }}$ tap and $\delta(t)$ is the Dirac delta function. Then, denoting the transmitted signal by $u$, the received version $r$ would be given by

$$
r[i]=u[i] \star h[i]+\omega[i],
$$

where $\star$ stands for convolution and $\omega[i]$ is additive thermal noise at the receiver. The cross-correlation between the received sequence $r[i]$ and the reference sequence, denoted by $R(\tau)$ is computed per subframe. To consider $N_{\text {NPRS }}$ consecutive NPRS occasions, the average of the set of cross-correlation estimates per positioning occasion is formed and denoted by $R_{\text {ave }}$. Finally, the first tap is estimated as

$$
\hat{\tau}=\arg \min _{\tau}\left\{\frac{R_{\mathrm{ave}}(\tau)}{\max \left(R_{\mathrm{ave}}\right)} \geq \zeta\right\},
$$

where $\zeta$ is the threshold value related to the multipath channel. The simulations of this thesis use $\zeta=0.5$, which implies half of the strongest peak, see [106]. In order to estimate the position using the OTDOA method, each UE must be linked to RSTD measurements of multiple eNBs. Let $\hat{\tau}^{(\ell)}$ correspond to the TOA from the $\ell^{\text {th }}$ eNB to the UE. Then,

$$
\hat{\tau}^{(i, j)}=\hat{\tau}^{(i)}-\hat{\tau}^{(j)},
$$

where $\hat{\tau}^{(i, j)}$ is the RSTD between eNB $j$ and the reference eNB $i$.

Let $h_{\mathrm{OTDOA}}(\theta)=r^{i}-r^{j},(i, j) \in K$, and $i \neq j$, be the OTDOA measurement model. Further, assume that $y^{(i, j)}$ contains the RSTD reports in (4.4), translated to relative distances, of the $K$ most powerful eNBs,

$$
y^{(i, j)}=h_{\mathrm{OTDOA}}(\boldsymbol{\theta})+e^{(i, j)},
$$

where the additive error term $e^{(i, j)}$ represents the measurement error times the speed of light. The performance of OTDOA positioning in NBIOT systems is evaluated using both snapshot estimation method and also in a filtering framework.

\subsection{Simulation study}

To evaluate the potential of optimizing resource consumption, RSTD reports are simulated to be sent to the mobile location center periodically or on on-demand. In the latter case, we further investigate two different scenarios. In one scenario, the main interest is to maintain a minimum accuracy level while reducing the resource consumption. In the second scenario, we allocate a certain maximal budget and try to find a reporting pattern that maximizes the final achievable accuracy. All different cases are introduced in Section 4.3.3. To increase the robustness of the filtering method, measurement noise outliers are detected using confidence bounds estimated from filter innovations. 


\subsubsection{Network deployment}

The simulated cellular network consists of 16 macro sites each having 3 cells, located in a hexagonal grid with an inter-site distance of $577 \mathrm{~m}$. The UE starts at a random position in the cellular network and passes through the network with different speeds. Figure 4.2 illustrates the cellular network deployment in which the red dots are macro sites, each with three cells, and the blue line represents the UE's trajectory. The UE trajectory represents a typical asset tracking use case, which is one important mobile IOT use case.

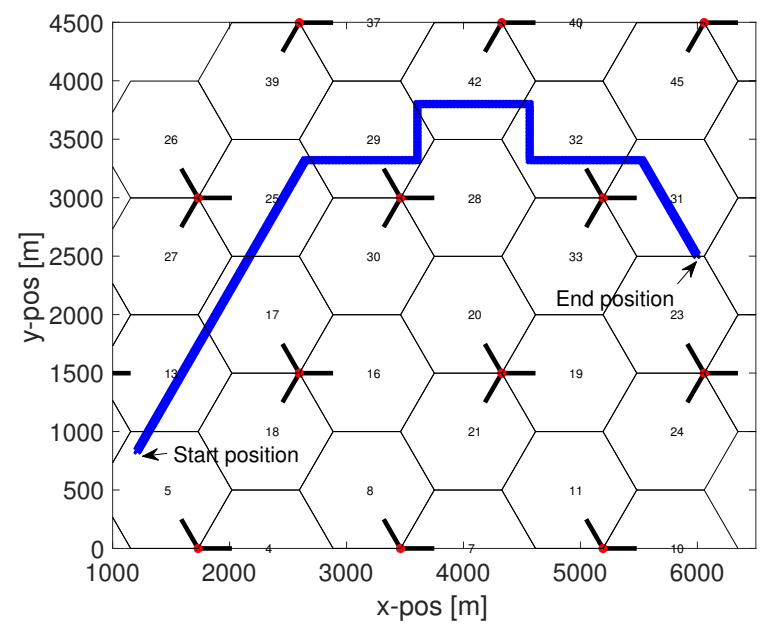

Figure 4.2: Deployment configurations of NBIOT. The blue line represents the UE trajectory [101, C2017 IEEE].

The radio distance wrapping technique, as described in [106], is used in this work. This technique simulates a network in which all sites are assumed to be surrounded by other sites. That is, all the UEs that might be on the border of the deployed network in Figure 4.2 are assured to be covered by other sites.

\subsubsection{RSTD report budget}

As described in Section 4.2.1, NBIOT supports a denser NPRS transmission scheme than PRS, allowing aggregation of data. This enhances TOA detection accuracy compared to other narrowband systems. The NBIOT system with an in-band configuration, as shown in Figure 1.1, within the $20 \mathrm{MHz}$ LTE standard is simulated in this work. The NPRS bandwidth is $180 \mathrm{KHz}$ where $N_{\text {NPRS }}=40$ subframes are transmitted every $T_{\mathrm{NPRS}}=160 \mathrm{~ms}$. The simulated NBIOT system parameters are given in Table 4.2 .

Based on the geometry of the user, hearability of cells, and channel conditions, we form the SNR vector containing signal strengths received from each cell. The cells below a given threshold are assumed to be out of coverage and the $K$ remain- 
Table 4.2: The deployed NBIOT parameters.

\begin{tabular}{ll}
\hline & Deployed NBIOT \\
\hline System carrier bandwidth & $20 \mathrm{MHz}$ \\
NBIOT configuration & In-band \\
NBIOT carrier bandwidth & $180 \mathrm{kHz}$ \\
Number of consecutive NPRS & 40 \\
NPRS period & $160 \mathrm{~ms}$ \\
\hline
\end{tabular}

ing ones with highest SNR form $N_{\text {rep }}=K-1$ TDOA measurements. In this setup, the reference cell is the one with highest SNR value.

Let $T_{\text {rep }}$ be the interval at which the UE reports RSTD measurements to the positioning center. In periodic reporting, the UE forms $N_{\text {rep }}$ RSTD estimates and reports it to the positioning center periodically every $T_{\text {rep }}$ seconds. Alternatively, in the on-demand reporting method, the UE decides the number of RSTDs depending on the SNR of the measured cells. In cases where the UE receives NPRS signals from strong cells, it forms more RSTDs to increase the accuracy. Weaker signals are assumed to correspond to the BSs further away from the UE that are more prone to severe measurement errors. In cases where the received signals are not strong enough, the UE reports less cells to lower the resource consumption.

\subsubsection{Positioning scenarios and budget constraints}

Let $\mathcal{K}$ be the set of BSs in the NBIOT cellular network; $\mathcal{K}=\{1, \ldots, N\}$. The $K$ most powerful BSs, measured in $\mathrm{dB}$, in the set $\mathcal{K}$ are used to form RSTD measurements. The parameter $K$, the number of BSs to be chosen, depends on a specific budget of reports. Hence, $K$ determines the number of BSs used in the position estimation method.

The modified Gauss-Newton method introduced in (3.7) is used for the static case while EKF (see Algorithm 2) is applied for the filtering scenario. RSTD measurements are performed with respect to a reference BS, resulting in a correlated measurement noise covariance matrix R. Diagonal and off-diagonal elements of $\mathbf{R}$ are similar to the ones reported in [71], to capture spatial correlations.

\section{Static case}

In the static case, we evaluate the obtainable position estimation accuracy using NPRS signals as a function of the number of measured cells. The unknown position of the UE is estimated using the modified Gauss-Newton method given by (3.7) initialized at the serving BS position.

\section{Dynamic case}

In this case we consider three different UE speeds 3,10 , and $30 \mathrm{~km} / \mathrm{h}$ modeled as a $\mathrm{CV}$ motion (3.22c). The unknown position and velocity of the UE are then tracked 
in an EKF filtering framework. The reporting budget per minute $\mathcal{B}$ is defined as $\mathcal{B}=\frac{60}{T_{\text {rep }}} \times N_{\text {rep }}$. Reports are either sent periodically or on an on-demand basis forming five different cases:

- Case 1: Two reports every two seconds, $\mathcal{B}=60$.

- Case 2: Three reports every three seconds, $\mathcal{B}=60$.

- Case 3: Five reports every four seconds, $\mathcal{B}=75$.

- Case 4: The UE increases the accuracy by forming more RSTD reports if the SNR values of available cells are above a certain threshold. However, it cannot exceed the maximum budget of $\mathcal{B}_{\max }=85$.

- Case 5: Varying number of reports every four seconds. The UE has a maximum budget $\mathcal{B}_{\text {max }}=45$ and depending on the SNR values, reports less or more cells to optimize resource consumption.

\subsection{Result and Conclusions}

\subsubsection{Simulation study}

The accuracy of different scenarios is numerically evaluated and the results are presented by the cumulative distribution function (CDF) of the root mean squared error (RMSE) of the horizontal accuracy.

Figure 4.3 illustrates the CDF of the horizontal positioning accuracy of the OTDOA estimates for the static case for the EPA and the ETU channel models, separately. Each curve is the result of 50 Monte Carlo simulation runs. Although the maximum positioning error is the same for both channels, the $90^{\text {th }}$ percentile of the error with 4 cells is around $76 \mathrm{~m}$ for the EPA channel and $495 \mathrm{~m}$ for the ETU.

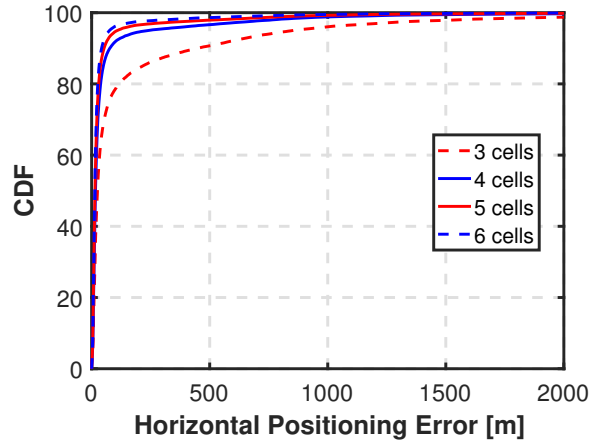

(a) Channel: EPA

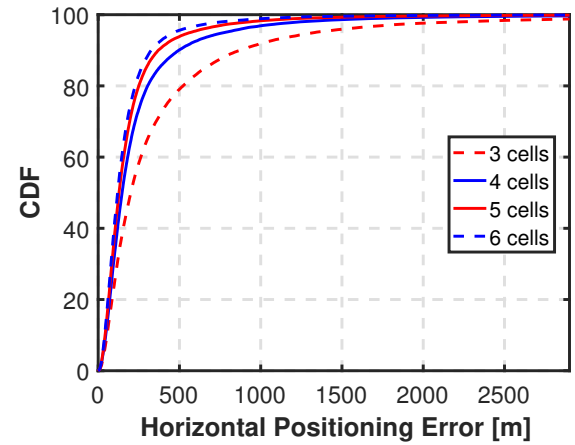

(b) Channel: ETU

Figure 4.3: The OTDOA performance for horizontal position accuracy of static case for both channel models [101, C2017 IEEE]. 
The large positioning errors in Figure 4.3 can be explained by multiple factors. There might be situations where less than three cells are heard. Although this can be handled using a tracking filter, the optimization method in the static case fails to converge as no unique optimum exists. NPRS signals received from cells belonging to the same site is another major influencing factor. Although it is mentioned that the UE uses measurements from unique sites, this is not always possible as there are situations when 3 unique sites are not within range. In cases where the serving cell, initial point, is far away from the true position, the final estimate can be a local minimum of the cost function. In the snapshot positioning method, where no temporal correlation is considered, good initialization is more important.

Figure 4.4 illustrates how poor initialization can be handled using tracking. The UE speed is $30 \mathrm{~km} / \mathrm{h}$ and RSTDs are sent as in case 1 . The shaded areas are the range of positioning errors obtained from 50 Monte Carlo runs and solid lines are the average positioning error.

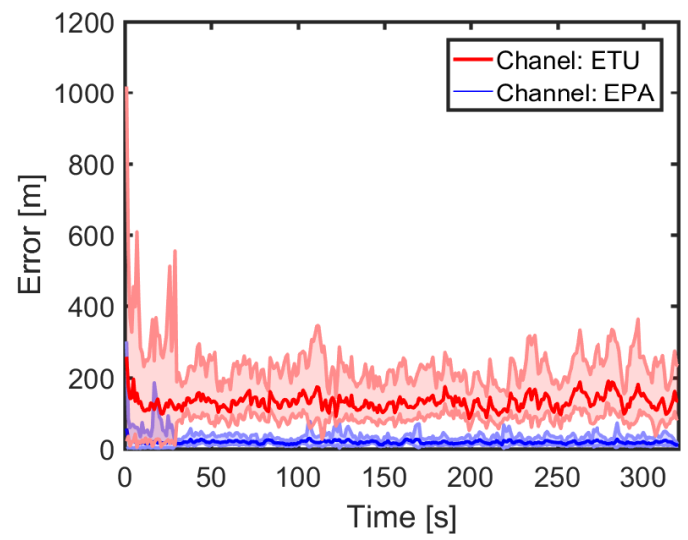

Figure 4.4: RMSE over time for NBIOT positioning using EKF for case 1 [101, C 2017 IEEE].

Figure 4.5 presents the performance of the periodic reporting methods, cases 2 and 3, introduced in Section 4.3.3 for different UE speeds. The figure suggests that in EPA channel models, more frequent reporting with less cells gives better results for all different speeds.

Cases 4 and 5 are compared to the periodic reporting for all cases and the results are presented in Table 4.3. For example, in the ETU channel, for a UE moving with $30 \mathrm{~km} / \mathrm{h}$, case 4 improves the $90^{\text {th }}$ percentile of positioning error from $238 \mathrm{~m}$ in case 3 to $183 \mathrm{~m}$ as seen in Figure 4.6b. Case 5 on the other hand, reduces reports per minute to almost a half while giving almost the same error characteristics. 


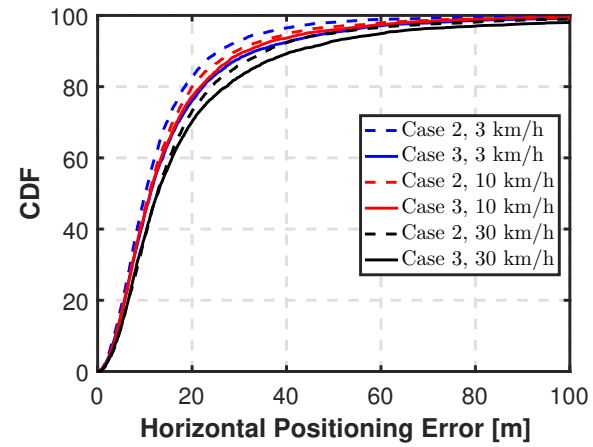

(a) Channel: EPA

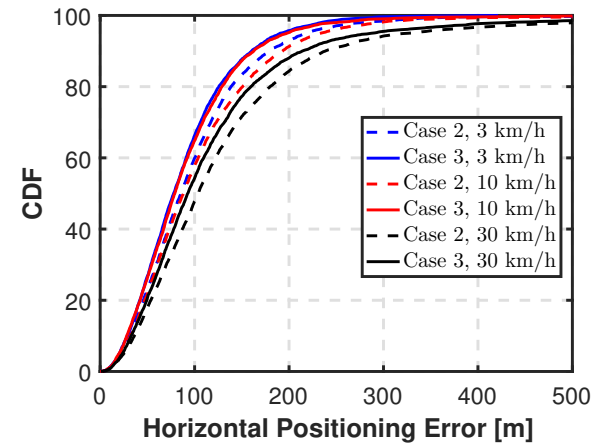

(b) Channel: ETU

Figure 4.5: The OTDOA performance for horizontal position accuracy of EKF for both channel models [101, C2017 IEEE].

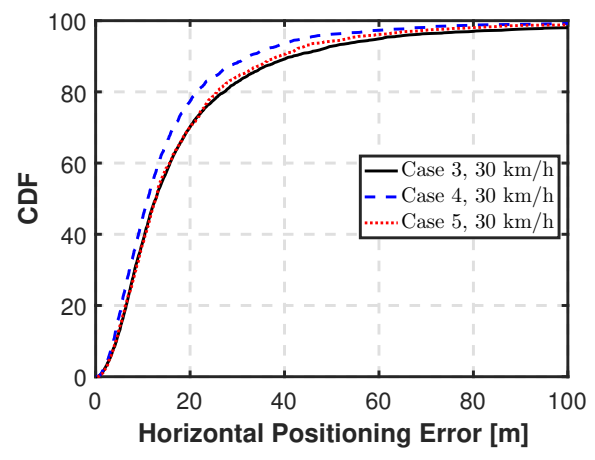

(a) Channel: EPA

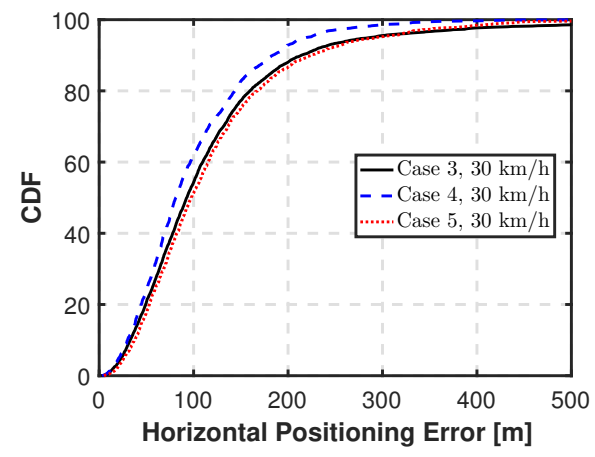

(b) Channel: ETU

Figure 4.6: Comparison of on-demand with periodic reporting schemes [101, C2017 IEEE].

\subsubsection{Conclusions}

The performance of device tracking in NBIOT systems has been investigated with respect to the horizontal positioning accuracy. The new transmission scheme of NPRS was used to compensate narrower bandwidth of the system and improve TOA estimates. Given a certain NPRS transmission scheme, the RSTD was formed and used for downlink OTDOA positioning.

The results were obtained for the EPA and ETU wireless channel models corresponding to low and high delay spread environments, respectively. We evaluated positioning in both static and dynamic cases. 
Table 4.3: Positioning error statistics and reporting budget $\mathcal{B}$ obtained by EKF for UE speeds $3 / 10 / 30 \mathrm{~km} / \mathrm{h}$ for both channel models .

\begin{tabular}{c|c|ccc|c}
\hline \multirow{2}{*}{ Channel } & \multirow{2}{*}{ Case } & \multicolumn{3}{|c|}{ RMSE [m] } & \multirow{2}{*}{ Budget } \\
\cline { 3 - 5 } & & Mean & $67 \%$ & $90 \%$ & \\
\hline \multirow{4}{*}{ EPA } & 1 & $14 / 15 / 18$ & $14 / 14 / 18$ & $26 / 27 / 35$ & 60 \\
& 2 & $13 / 15 / 19$ & $14 / 15 / 17$ & $25 / 29 / 36$ & 60 \\
& 3 & $16 / 15 / 20$ & $16 / 15 / 18$ & $33 / 31 / 41$ & 75 \\
& 4 & $12 / 13 / 16$ & $11 / 13 / 15$ & $22 / 26 / 32$ & $75-85$ \\
& 5 & $15 / 19 / 19$ & $14 / 16 / 18$ & $29 / 34 / 38$ & $35-45$ \\
\hline \multirow{6}{*}{ ETU } & 1 & $104 / 111 / 131$ & $115 / 118 / 145$ & $180 / 195 / 244$ & 60 \\
& 2 & $99 / 105 / 135$ & $111 / 116 / 137$ & $178 / 193 / 238$ & 60 \\
& 3 & $87 / 95 / 117$ & $101 / 103 / 124$ & $160 / 162 / 213$ & 75 \\
& 4 & $71 / 80 / 97$ & $80 / 94 / 110$ & $127 / 142 / 183$ & $75-85$ \\
& 5 & $100 / 118 / 118$ & $113 / 128 / 129$ & $188 / 230 / 223$ & $35-45$ \\
\hline
\end{tabular}





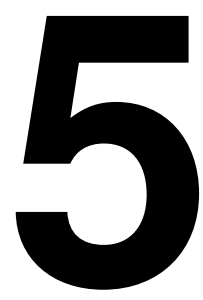

\section{Positioning Using Fusion of TOF and TDOA}

It is often presumed in UE-assisted positioning that at least three different BSs must be measured in order to localize a target uniquely. However, there might be situations where information associated to three BSs is unavailable. For example, the UE may only detect less than three base stations. In cases where signals from only two BSs are detectable, the UE cannot be localized uniquely with an acceptable level of uncertainty using conventional methods for snapshot positioning.

This thesis proposes an approach based on fusion of TOF and TDOA measurements gathered from two BSs over a time series. Solving the set of nonlinear equations for the 2-D position in noise-free scenarios gives two possible closedform solutions for the UE position. The derived solutions are used to estimate the snapshot position of the UE for realistic measurement model using the unscented transformation. The estimated positions of the unscented transformation are then fed to a bank of Kalman filters as position measurements of the UE. While the UE passes through the cellular network, it will be handed over to other BSs based on an event-triggered handover procedure. As soon as a handover occurs, the weights of unlikely modes in the filter bank get noticeably smaller relative to the likely modes. The ambiguity in the positions can then be resolved as soon as a change in the set of BSs occurs.

In the remainder of this chapter we first provide background and related work of timing-based and hybrid localization methods in Section 5.1. The results of the simulation study and real data experiments are given in Section 5.2.

\subsection{Background}

Estimating the 2-D location of a UE from TOA measurements, either forming TOF or TDOA measurements, associated to at least three BSs is a well studied problem. One typical example is to position the UE due to an emergency call, where 
the UE is requested to report TDOA measurement associated to detected base stations. With this snapshot measurement report, the network needs measurements associated to at least three base stations.

To increase the location estimation accuracy, hybrid approaches are introduced in the literature. In a majority of radio-based hybrid positioning approaches, AOA measurements are used together with TDOA [30] and TOA [87, 98]. However, collecting AOA measurements in practical applications is not always trivial. The angle at which a signal arrives at the receiver can be computed either by using a directional antenna or by inferring it indirectly from TOA measurements using antenna arrays $[29,75,120]$. Since directional antennas with suitable size and cost are generally difficult to realize, antenna arrays are more commonly used. However, as shown in [112], the position accuracy obtained by TOA measurements cannot be further improved by AOA measurements obtained from antenna arrays.

Although hybrid positioning solutions can be of great use in cases where the number of available BSs are limited, all these studies assume that at least three BSs are available. Positioning using measurements gathered from only two base stations has not been widely studied until very recently. In [124], the location of a fixed object is estimated using TOA and AOA measurements collected from two BSs. The algorithm is only valid in circular scattering environments and need to be tailored for each individual scenario with various scattering radius properties. Another recent study in [132], uses one TDOA and two AOA measurements collected from two base stations for passive localization of one static source. Although a localization error of around $300 \mathrm{~m}$ (for AOA noise standard deviation of two degrees) might be acceptable in some static source localization applications, in dynamic scenarios, there is no chance to estimate the velocity and other properties of the UE. The authors in [64] investigate the position estimation of a vessel in an automatic identification system using TOA and TDOA measurements. The performance of the estimator is evaluated in terms of geometric dilution of precision and no realistic noisy measurements is tested with the proposed method.

In this work, BSs to which range is measured at each time instant change along the trajectory. Contrary to the NBIOT scenario where the SNR values were used to form the serving and non-serving BSs, we here assume that the serving BS is the BS that has the smallest geometric distance to the UE. Similarly, the neighboring BS is defined to be the BS which, geometrically is, the second closest to the UE. With these assumptions, it is possible to define areas identifying which BS is providing TOF measurements and which pair of BSs are detected for TDOA measurements as shown in Figure 5.1a and Figure 5.1b, respectively. Interestingly, the areas for TOF measurements define hexagonal cells, while the areas for the detected BS pairs for TDOA measurements define parallelograms (e.g., the area where BS $S_{1}$ and $S_{5}$ are detected for the TDOA measurement is defined as the parallelogram having corners defined by $S_{1}$ and $S_{5}$. In this setup, $S_{1}$ provides the TOF measurement, and the TDOA is measured based on signals from $S_{1}$ and $S_{5}$ ).

In the simulation study reported in Section 5.2.1, we consider that the UE is moving on a predefined trajectory, which has a flower-shape structure as depicted in Figure 5.1. The serving BS and the neighboring BSs involved in the positioning 


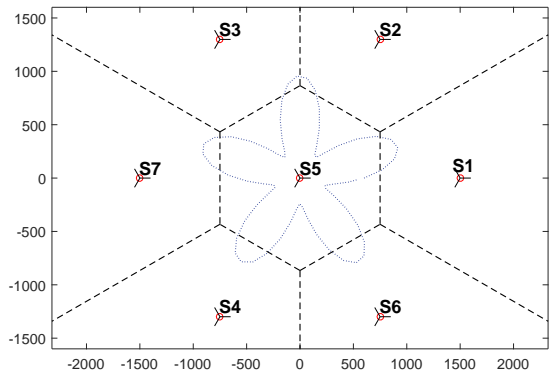

(a) TOF

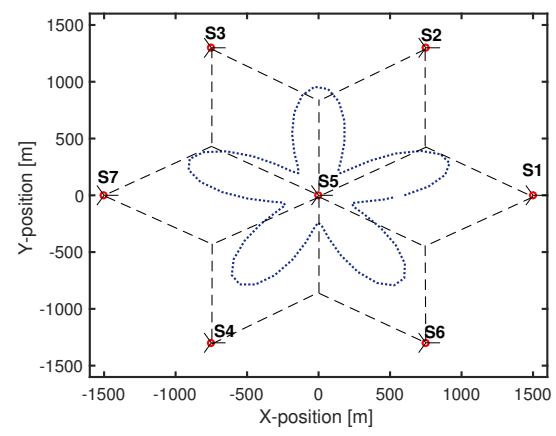

(b) TDOA

Figure 5.1: Simulation scenario with flower-shaped trajectory, and areas identifying (a) which BS is providing TOF measurements, and (b) which pair of BSs is detected for TDOA measurements

process will change depending on the current location of the UE.

\subsubsection{Static case}

Let $r_{1}$ denote the distance between the unknown position of the UE, denoted by $\theta$, to the serving cell $B S 1$ and $r_{2}$ the distance to the neighboring cell $B S 2$. The noise-free absolute and relative ranges are $2 r_{1}$ and $r_{21}=r_{2}-r_{1}$, respectively. Further, let $\boldsymbol{g}\left(r_{1}, r_{21}\right)$ be the inverse mapping that relates the unknown position UE to the range measurements, $\boldsymbol{\theta}=\boldsymbol{g}\left(r_{1}, r_{21}\right)$. In the rest of this section, we first derive closed form solutions of the UE's position given $r_{1}$ and $r_{21}$. The available TOF and TDOA measurements are noisy measurements of true distances $2 r_{1}$ and $r_{21}=r_{2}-r_{1}$, used to estimate the UE's position.

\section{Noise free case}

To facilitate the derivation, the first step is to temporarily use a local coordinate system, where the two BSs, $S_{1}$ and $S_{2}$, are symmetrically located at the $x$-axis as illustrated in Figure 5.2. Define a rotation $R$ and translation $t$ such that, $\bar{\theta}=$ $\boldsymbol{R} \boldsymbol{\theta}+\boldsymbol{t}$ and $\overline{\boldsymbol{\ell}}_{i}=\boldsymbol{R} \boldsymbol{\ell}_{i}+\boldsymbol{t}$, where $\overline{\boldsymbol{\ell}}_{1}=(-D / 2,0)^{T}$ and $\overline{\boldsymbol{\ell}}_{2}=(D / 2,0)^{T}$. We define the solution as $\overline{\boldsymbol{\theta}}=\overline{\boldsymbol{g}}\left(r_{1}, r_{21}\right)$. Since the change back to global coordinates is given by $\boldsymbol{\theta}=\boldsymbol{R}^{T}(\overline{\boldsymbol{\theta}}-\boldsymbol{t})$, the desired mapping is given by $\boldsymbol{\theta}=\boldsymbol{g}\left(r_{1}, r_{21}\right)=\boldsymbol{R}^{T}\left(\overline{\boldsymbol{g}}\left(r_{1}, r_{21}\right)-\boldsymbol{t}\right)$. Note that the rotation matrix $\boldsymbol{R}$ does not affect scale, so the distances $r_{i}$ are the same in local coordinates, and the BS separation is also the same in global and local coordinates.

Geometrically, the solution in the noise-free case is given by the intersection 


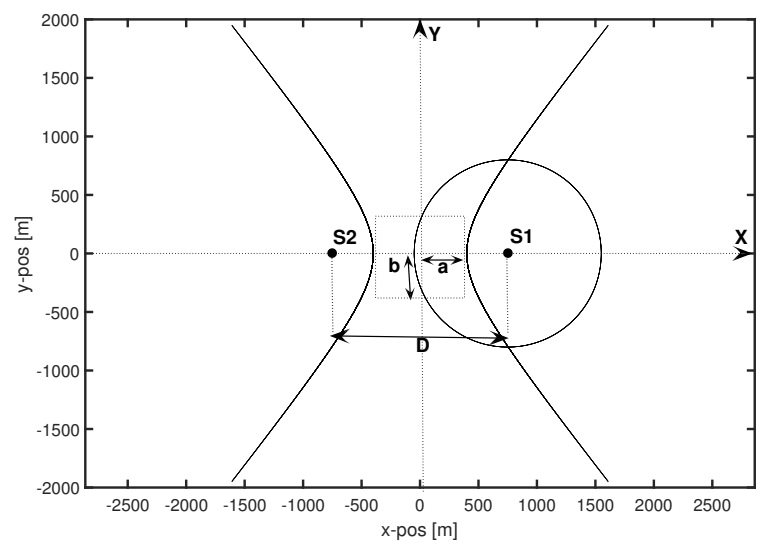

Figure 5.2: Equivalent local coordinate system for the two BS scenario [100, C) 2016 IEEE].

of a circle and a hyperbola, defined by

$$
\begin{aligned}
\left(\bar{\theta}_{x}-\frac{D}{2}\right)^{2}+\bar{\theta}_{y}^{2} & =r_{1}^{2}, \\
\frac{\bar{\theta}_{x}^{2}}{a^{2}}-\frac{\bar{\theta}_{y}^{2}}{b^{2}} & =1,
\end{aligned}
$$

where $a^{2}=\frac{1}{4} r_{21}^{2}$ and $b^{2}=\frac{1}{4}\left(D^{2}-r_{21}^{2}\right)$. Algebraically, the two intersections are given by

$$
\overline{\boldsymbol{\theta}}=\left\{\begin{array}{cc}
{\left[\begin{array}{c}
\bar{g}_{x}\left(r_{1}, r_{21}\right) \\
\delta \bar{g}_{y}\left(r_{1}, r_{21}\right)
\end{array}\right],} & r_{1}+r_{2}>D \\
{\left[\begin{array}{cc}
\bar{g}_{x}\left(r_{1}, r_{21}\right) \\
0
\end{array}\right],} & r_{1}+r_{2}=D \\
\emptyset, & \text { otherwise }
\end{array}\right.
$$

with

$$
\begin{aligned}
& \bar{g}_{x}\left(r_{1}, r_{21}\right)=\frac{r_{21}\left(r_{21}+2 r_{1}\right)}{2 D}, \\
& \bar{g}_{y}\left(r_{1}, r_{21}\right)=\frac{\sqrt{\left(D^{2}-r_{21}^{2}\right)\left(\left(2 r_{1}+r_{21}\right)^{2}-D^{2}\right)}}{2 D},
\end{aligned}
$$

where $\delta \in\{ \pm 1\}$ is a discrete parameter representing the two possible intersection points. Note that the circle and hyperbola can intersect in no, one or two points, where two solutions is the normal case, and only having one solution is a degenerated case when $r_{1}+r_{2}=D$ and the solution lies on the $x$ axis. The case of no 
solution, corresponding to $r_{1}+r_{2}<D$, can happen for noisy measurements and may need special care.

\section{Noisy case}

Let $e_{\mathrm{dl}, 1}$ and $e_{\mathrm{ul}, 1}$ denote the measurement noises of the serving $\mathrm{BS}$ in the downlink and uplink directions, respectively. The measurement noise of the second BS has only contributions from the downlink and is denoted by $e_{\mathrm{dl}, 2}$. The noisy TOF and TDOA measurement models are thus defined by

$$
\begin{aligned}
& z_{1}=2 r_{1}+e_{\mathrm{dl}, 1}+e_{\mathrm{ul}, 1}, \\
& z_{2}=r_{2}-r_{1}+e_{\mathrm{dl}, 2}-e_{\mathrm{dl}, 1},
\end{aligned}
$$

where $e_{\mathrm{dl}, i} \sim \mathcal{N}\left(0, \sigma_{\mathrm{dl}}^{2}\right)$ and $e_{\mathrm{ul}, 1} \sim \mathcal{N}\left(0, \sigma_{\mathrm{ul}}^{2}\right)$. We define the measurement vector $z=\left(z_{1}, z_{2}\right)^{T}$ where $z \sim \mathcal{N}\left(\left(2 r_{1}, r_{21}\right)^{T}, \boldsymbol{R}\right)$ with $\boldsymbol{R}$ given by

$$
\boldsymbol{R}=\operatorname{Cov}(\boldsymbol{z})=\left[\begin{array}{cc}
\sigma_{\mathrm{dl}}^{2}+\sigma_{\mathrm{ul}}^{2} & -\sigma_{\mathrm{dl}}^{2} \\
-\sigma_{\mathrm{dl}}^{2} & 2 \sigma_{\mathrm{dl}}^{2}
\end{array}\right] .
$$

Let $\boldsymbol{e}=\left(e_{\mathrm{dl}, 1}+e_{\mathrm{ul}, 1}, e_{\mathrm{dl}, 2}-e_{\mathrm{dl}, 1}\right)^{T}$ be the measurement noise vector. The stochastic vector $(\mathbf{z}-\mathbf{e})$ can be related to the UE position through a nonlinear mapping $\boldsymbol{\theta}(\delta)=\boldsymbol{g}(\mathbf{z}-\mathbf{e}, \delta)$, mapping $\mathbb{R}^{2} \rightarrow \mathbb{R}^{2}$. Nonlinearities in $\boldsymbol{g}(\cdot)$ mean that the corresponding position will be non-Gaussian distributed. Hence, the UE position estimation problem turns into the problem of efficiently approximating the mean and covariance of Gaussian random variables that have been transformed through nonlinearities.

In the following, we let $\hat{\theta}(\delta)$ denote the UE position estimate. There is a vast literature on how to treat nonlinearities and compute $\hat{\theta}(\delta)$. First and second order Gaussian approximations are based on Taylor series expansions to approximate a linear model. For example, a first-order Taylor approximation of the nonlinear mapping $g(z-e, \delta)$ around the measurement vector $z$ is given by

$$
\boldsymbol{g}(z-\boldsymbol{e}, \delta) \approx \boldsymbol{g}(z, \delta)-\boldsymbol{g}^{\prime}(z, \delta) \boldsymbol{e},
$$

where $g^{\prime}(\cdot)$ is the gradient of $\boldsymbol{g}(\cdot)$ with respect to $z$. From this linear approximation, we obtain the mean and covariance which is sometimes referred to as Gauss' approximation formula, yielding

$$
\begin{aligned}
& \hat{\boldsymbol{\theta}}(\delta)=\mathbb{E}_{p(e)} \boldsymbol{g}(z-\boldsymbol{e}, \delta) \approx \boldsymbol{g}(\boldsymbol{z}, \delta) \\
& \boldsymbol{P}_{\boldsymbol{\theta}(\delta)}=\operatorname{Cov}(\boldsymbol{g}(z-\boldsymbol{e}, \delta)) \approx \boldsymbol{g}^{\prime}(z, \delta) \boldsymbol{R}\left(\boldsymbol{g}^{\prime}(z, \delta)\right)^{T}
\end{aligned}
$$

Thus, the position estimator $\hat{\theta}(\delta)$ is rather simple, as it only replaces the noisefree measurements $r_{1}$ and $r_{21}$ by the noisy measurements $z$.

The Unscented transform (UT) is another method that differs from the Taylorseries-based in the sense that it does not approximate the nonlinear function, rather it tries to directly approximate the first two statistical moments.

UT approximates $\theta(\delta)=\boldsymbol{g}(\boldsymbol{z}-\boldsymbol{e}, \delta)$ and its associated uncertainty as the first two moments of a Gaussian distribution, $\mathcal{N}(\boldsymbol{\theta}(\delta), \boldsymbol{\Lambda}(\delta))$. The approximation process is given by Algorithm 3, see [54], [107] for detailed explanation. 


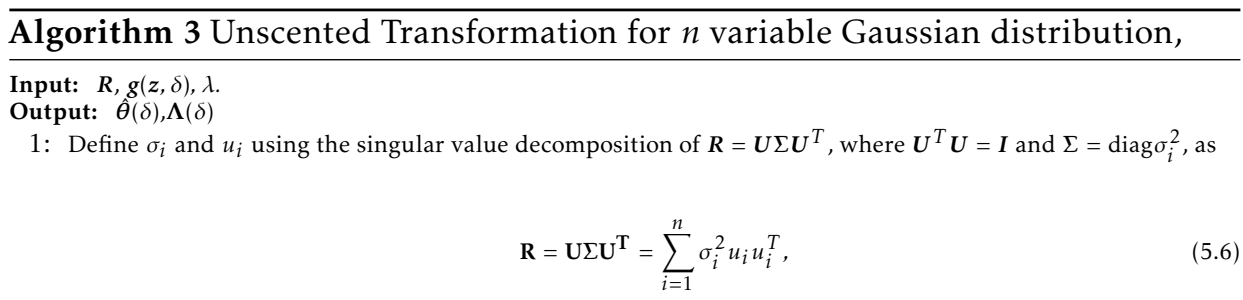

where $u_{i}$ is the $i$ :th column of $\boldsymbol{U}$ and $\sigma_{i}^{2}=\Sigma_{i, i}$ is the $i$ :th diagonal element of $\Sigma$.

2: Form a set of $2 n+1$ sigma points

$$
\begin{aligned}
\tau^{(0)} & =0, \\
\tau^{( \pm i)} & = \pm \sqrt{2+\lambda} \sigma_{i} u_{i}, \quad i=1, \ldots, n .
\end{aligned}
$$

where $\lambda$ is a design parameter.

3: Map the sigma points to $\widetilde{\boldsymbol{\theta}}^{(n)}(\delta)=\boldsymbol{g}\left(z-\boldsymbol{\tau}^{(n)}, \delta\right)$ by propagating them through nonlinear function $\boldsymbol{g}($.$) for n \in$ $\{0, \pm i\}$.

4: Compute the constant weights $w_{n}$

$$
\begin{aligned}
w^{(0)} & =\frac{\lambda}{2+\lambda}, \\
w^{( \pm i)} & =\frac{1}{2(2+\lambda)}, \quad i=1, \ldots, n .
\end{aligned}
$$

5: Estimate the mean and covariance of the transformed variable from the mapped sigma points

$$
\begin{aligned}
\mathrm{E}[\boldsymbol{g}(\boldsymbol{z}, \delta)] \approx \hat{\boldsymbol{\theta}}(\delta) & =\sum_{j \in n} w^{(j)} \widetilde{\boldsymbol{\theta}}^{(j)}(\delta) \\
\operatorname{Cov}[\boldsymbol{g}(\boldsymbol{z}, \delta)] \approx \boldsymbol{\Lambda}(\delta) & =\sum_{j \in n} w^{(j)}\left(\widetilde{\boldsymbol{\theta}}^{(j)}(\delta)-\boldsymbol{\theta}(\delta)\right) \\
& \times\left(\widetilde{\boldsymbol{\theta}}^{(j)}(\delta)-\boldsymbol{\theta}(\delta)\right)^{T} .
\end{aligned}
$$

\subsubsection{Dynamic case}

In the dynamic case, we would like to use filtering methods to solve the problem. Given the sequence of $\delta$, this can be done using a KF. However, as $\delta$ is unknown, we resort to enumerating possible $\delta$ combinations, and keeping track of how likely they are. This is known as a filter bank. Since the number of possible mode combinations in the filter bank grows exponentially, the problem becomes intractable over time. Hence, to make the algorithm tractable, the number of mode combinations need to be reduced. Next, a filter bank approach will be described with the model structure.

\section{Filter bank solution}

We use a bank of Kalman filters to compute the filtering distribution of the jump Markov state-space model. Applying the filter bank assures keeping track of both possible modes of the system along the trajectory. Then, it is possible to avoid making a hard decision until the set of involved BSs changes. 
To better illustrate how a change in the pair of BSs introduces two solutions, see Figure 5.3 in which the two possible solutions are marked with blue and black dots while the UE goes through the network. Initiating at time 0 , the UE starts its path at point $(600,0)^{T}$. The serving BS is BS5 and the neighboring BS is BS1. Let $\tilde{t}$ be the time at which the first handover occurs and the new set of BSs change to BS5 and BS2. Two possible positions at time $\tilde{t}-1$ are $(690,390)^{T}$ and $(690,-390)^{T}$ while the former is the true position of the UE.

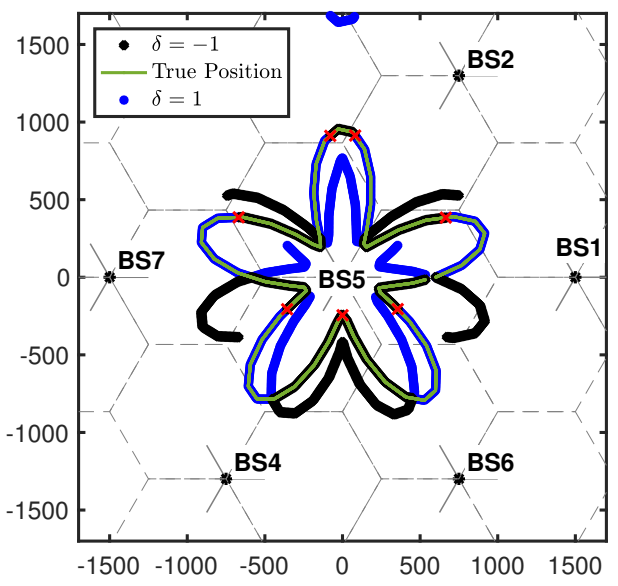

Figure 5.3: Simulated network deployment with seven BSs and the true trajectory, marked with green, together with the true and ambiguous solutions, marked with blue for $\delta=1$ and black for $\delta=-1$. Time instance at which the measured BS pair change are marked with red crosses.

Using the additional information obtained at the time of handovers, the true sequence of modes gets a higher weight relative to the other set of mode making it possible to keep the relevant estimates and discard the rest. The method is illustrated by the flow diagram given in Figure 5.4.

\section{Jump Markov state-space model}

The estimated positions $\hat{\boldsymbol{\theta}}(\delta)$ and their associating covariance matrices $\Lambda(\delta)$ given by (5.9) are used as the measurements $y_{t}$ in the state-space model. The motion of the UE is modeled using the CV model (3.22c) translational kinematics where the unknown inputs to the system are modeled as process noise. The (Kalman filter 


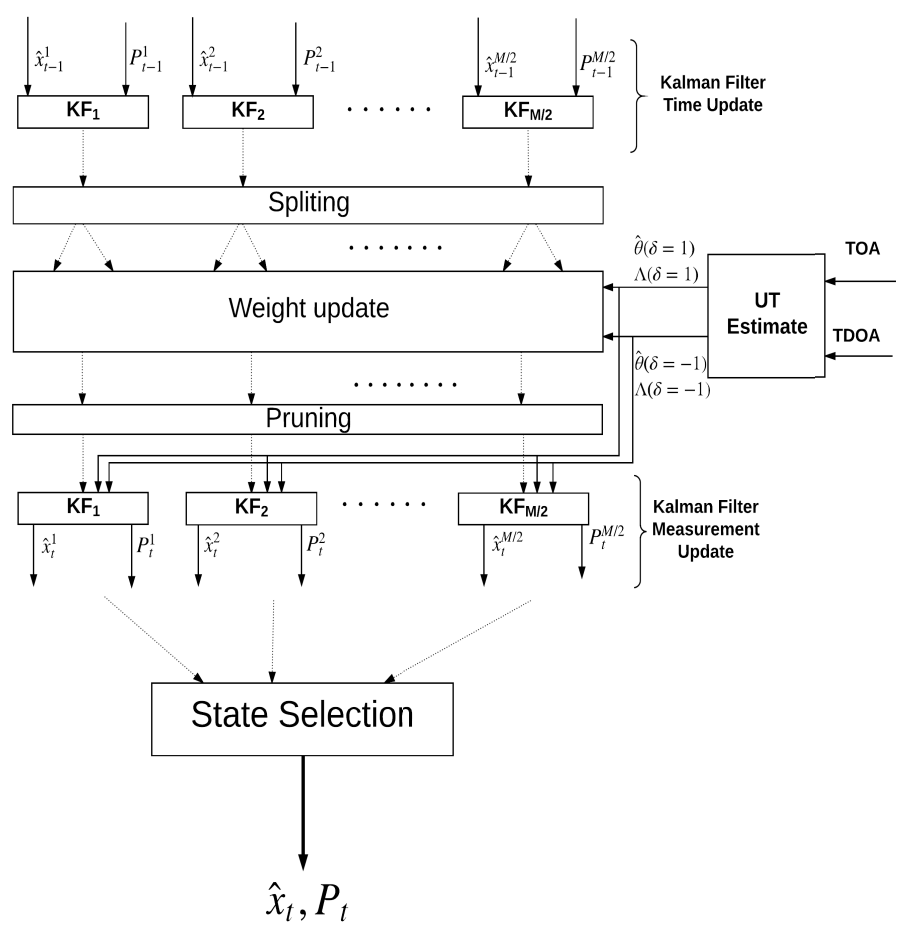

Figure 5.4: Flow diagram of the proposed method.

bank) model is defined by

$$
\begin{aligned}
\boldsymbol{x}_{t+1} & =\boldsymbol{F} \boldsymbol{x}_{t}+\omega_{t+1}, \\
\mathbf{0} & =\boldsymbol{H} \boldsymbol{x}_{t}+\boldsymbol{c}_{t}\left(\delta_{t}\right)+\boldsymbol{e}_{t}\left(\delta_{t}\right), \\
\omega_{t+1} & \sim \mathcal{N}(\mathbf{0}, \mathbf{Q}), \\
\boldsymbol{e}_{t}\left(\delta_{t}\right) & \sim \mathcal{N}\left(\mathbf{0}, \boldsymbol{\Lambda}_{t}\left(\delta_{t}\right)\right), \\
\mathrm{p}\left(\delta_{t+1} \mid \delta_{t}\right) & =\boldsymbol{\Pi}_{t}^{\delta_{t+1}, \delta_{t}},
\end{aligned}
$$

with

$$
\begin{aligned}
\boldsymbol{F} & =\left(\begin{array}{cc}
\mathbf{I}_{2} & T \mathbf{I}_{2} \\
\mathbf{0} & \mathbf{I}_{2}
\end{array}\right), \quad \boldsymbol{H}=\left(\begin{array}{cccc}
1 & 0 & 0 & 0 \\
0 & 1 & 0 & 0
\end{array}\right), \\
\boldsymbol{c}_{t}\left(\delta_{t}\right) & =-\hat{\boldsymbol{\theta}}_{t}\left(\delta_{t}\right)=-\boldsymbol{y}_{t}, \quad \boldsymbol{Q}=\sigma_{\omega}^{2}\left(\begin{array}{c}
\frac{T^{2}}{2} \mathbf{I}_{2} \\
T \mathbf{I}_{2}
\end{array}\right)\left(\begin{array}{c}
\frac{T^{2}}{2} \mathbf{I}_{2} \\
T \mathbf{I}_{2}
\end{array}\right)^{\mathrm{T}},
\end{aligned}
$$

where $\sigma_{\omega}$ is the process noise standard deviation, $T$ is the sampling interval, and $\Pi_{t}^{\delta_{t+1}, \delta_{t}}$ models the mode transition probability at time $t$. The transition probabil- 
ities are modeled as a first-order Markov chain where $\mathrm{p}\left(\delta_{t} \mid \delta_{t-1}, \delta_{t-2}, \ldots, \delta_{1}\right)=$ $\mathrm{p}\left(\delta_{t} \mid \delta_{t-1}\right)$.

\section{Kalman filter bank}

The posterior distribution of states $x_{t}, t \in\{1: N\}$ is a mixture of $2^{N}$ distributions. Enumerating each unique sequence of modes by $\delta_{1: t}^{(i)}$, the posterior distribution is given by

$$
p\left(x_{t} \mid y_{1: t}\right)=\sum_{i=1}^{2^{t}} w_{t}^{(i)} \cdot p\left(x_{t} \mid \delta_{1: t}^{(i)}, y_{1: t}\right)
$$

where for $i=2^{t}$ number of unique sequences of modes, $\delta_{1: t}^{(i)}=\left\{\delta_{j}^{(i)}\right\}_{j=1}^{t}$, the mixing probability $w_{t}^{(i)}$ is defined as $w_{t}^{(i)} \triangleq p\left(\delta_{1: t}^{(i)} \mid y_{1: t}\right)$. Using the Markovian property of modes, the mixing probabilities in the mixture distribution is given by

$$
w_{t}^{(i)} \propto w_{t-1}^{(i)} \Pi_{t}^{\delta_{t}^{(i)}, \delta_{t-1}^{(i)}} \mathcal{N}\left(y_{t} \mid \hat{y}_{t}^{(i)}, \boldsymbol{S}_{t}^{(i)}\right)
$$

where $S_{t}$ is the innovation covariance computed by the Kalman filter applied to each individual mode. The complete filter bank, as introduced above, grows exponentially in the number of filters, as time evolves, leading to the computation of the posterior distribution to become intractable. To overcome this issue, one needs to reduce the size of the filter bank.

\section{Mode reduction}

Mode reduction can be performed by introducing a pruning or merging step on the tree of mode sequences. Generally, there are different ways for pruning and/or merging in online algorithms, see for example [60],[54]. In this work, a filter bank with pruning of low-probability branches is applied to compute the posterior distribution of the branches with highest probabilities only. To do so, a sequence-splitting step is defined, in which the existing high-probability branches are extended with all possible modes at each time instance. In the next step, weights of all the newly constructed branches are updated and only $M$ branches with highest probabilities are kept and the rest are discarded. The remaining branches are then updated in a measurement update phase. The whole algorithm for the filter bank with pruning is given in Algorithm 4.

\section{State selection}

The result of running parallel filters introduced in Algorithm 4 can be used to construct a set of quadruples $\left\{\delta_{1: t}, \hat{\boldsymbol{x}}_{1: t}(\delta), \hat{\boldsymbol{P}}_{1: t}(\delta), w_{1: t}(\delta)\right\}$. The surviving modes include both the desired solution, the UE position, and the second solution of the 


\section{Algorithm 4 Kalman filter bank with pruning}

Input: $x_{0}, P_{0}, y_{1: \mathrm{N}}$

Output: $\hat{\boldsymbol{x}}_{t \mid t}^{(i)}, \boldsymbol{P}_{t \mid t}^{(i)}, \delta_{1: t}^{(i)}, w_{t}^{(i)}$ for $i \in\{1: M\}$.

$$
\begin{aligned}
& \text { Initialization: } \\
& \delta_{1: 0}^{(k)}=\emptyset, \hat{\boldsymbol{x}}_{0 \mid 0}^{(k)}=x_{0}, P_{0 \mid 0}^{(k)}=\boldsymbol{P}_{0}, w_{0}^{(k)}=1 \text { for } k=1 .
\end{aligned}
$$

1: for $t=1$ to $\mathrm{N}$ do

2: $\quad$ State time update: for $i=1, \ldots, k$

$$
\begin{aligned}
& \hat{\boldsymbol{x}}_{t \mid t-1}^{(i)}=\boldsymbol{F} \hat{\boldsymbol{x}}_{t-1 \mid t-1}^{(i)}, \\
& \boldsymbol{P}_{t \mid t-1}^{(i)}=\boldsymbol{F} \boldsymbol{P}_{t-1 \mid t-1}^{(i)} \boldsymbol{F}^{\mathrm{T}}+\boldsymbol{Q}_{t \mid t} .
\end{aligned}
$$

3: Splitting: for all $\left\{\delta_{1: t-1}^{(i)}\right\}_{i=1}^{k}$ construct:

$$
\begin{aligned}
\delta_{1: t-1}^{(i,-1)} & =\left\{\delta_{1: t-1}^{(i)},-1\right\} \\
\delta_{1: t-1}^{(i,+1)} & =\left\{\delta_{1: t-1}^{(i)},+1\right\} \\
k & =2 k
\end{aligned}
$$

4: $\quad$ Weight update: for $i=1, \ldots, k$

$$
\begin{aligned}
& \widetilde{w}_{t}^{(i)}=w_{t-1}^{(i)} \Pi_{t}^{\delta_{t}^{(i)}}, \delta_{t-1}^{(i)} \mathcal{N}\left(y_{t} \mid \hat{y}_{t}^{(i)}, s_{t}^{(i)}\right), \\
& w_{t}^{(i)}=\frac{\widetilde{w}_{t}^{(i)}}{\sum_{j=1}^{M} \widetilde{w}_{t}^{(j)}} .
\end{aligned}
$$

5: $\quad$ Pruning: (if $k>M$ )

Sort the weights in descending order, $w_{t}^{(1)} \geq w_{t}^{(2)} \geq \ldots \geq w_{t}^{(M)} \ldots$. Keep the first $M$ sequences and discard the rest.

$$
k=M
$$

6: $\quad$ State measurement update: for $i=1, \ldots, k$

$$
\begin{aligned}
& \boldsymbol{S}_{t}^{(i)}=\boldsymbol{H} \boldsymbol{P}_{t \mid t-1}^{(i)} \boldsymbol{H}^{\mathrm{T}}+\boldsymbol{\Lambda}_{t}\left(\delta_{t}^{(i)}\right), \\
& \boldsymbol{K}_{t}^{(i)}=\boldsymbol{P}_{t \mid t-1}^{(i)} \boldsymbol{H}^{\mathrm{T}}\left(\boldsymbol{s}_{t}^{(i)}\right)^{-1}, \\
& \hat{\boldsymbol{x}}_{t \mid t}^{(i)}=\hat{\boldsymbol{x}}_{t \mid t-1}^{(i)}+\boldsymbol{K}_{t}^{(i)}\left(y_{t}-\boldsymbol{H} \hat{\boldsymbol{x}}_{t \mid t-1}^{(i)}\right), \\
& \boldsymbol{P}_{t \mid t}^{(i)}=\boldsymbol{P}_{t \mid t-1}^{(i)}-\boldsymbol{K}_{t}^{(i)} \boldsymbol{H} \boldsymbol{P}_{t \mid t-1}^{(i)} .
\end{aligned}
$$

7: end for

8: return $\hat{x}_{t \mid t}^{(i)}, P_{t \mid t}^{(i)}, \delta_{1: t}^{(i)}, w_{t}^{(i)}$. 
intersection problem. The state selection stage of Figure 5.4 uses additional information gained at each time instance of handover to provide a single sequence of state estimates together with their associated uncertainties.

Figure 5.5 illustrates the first 19 seconds of the algorithm applied on the simulated network with $M=2$. As the figure suggests, the mode sequence $\delta_{1: 18}^{(1)}$ contains the UE's estimated positions and $\delta_{1: 18}^{(-1)}$ contains the shadow solutions. At $t=19$, the set of invovled BSs change, hence $\delta_{19}^{(1)}$ gets a large weight. At this point, the whole sequence $\delta_{1: 18}^{(-1)}$ is discarded and the mode history for both filters becomes $\delta_{1: 18}^{(1)}$. The branches are then extended with both possible modes at $t=19$ and the same procedure is repeated in the rest.

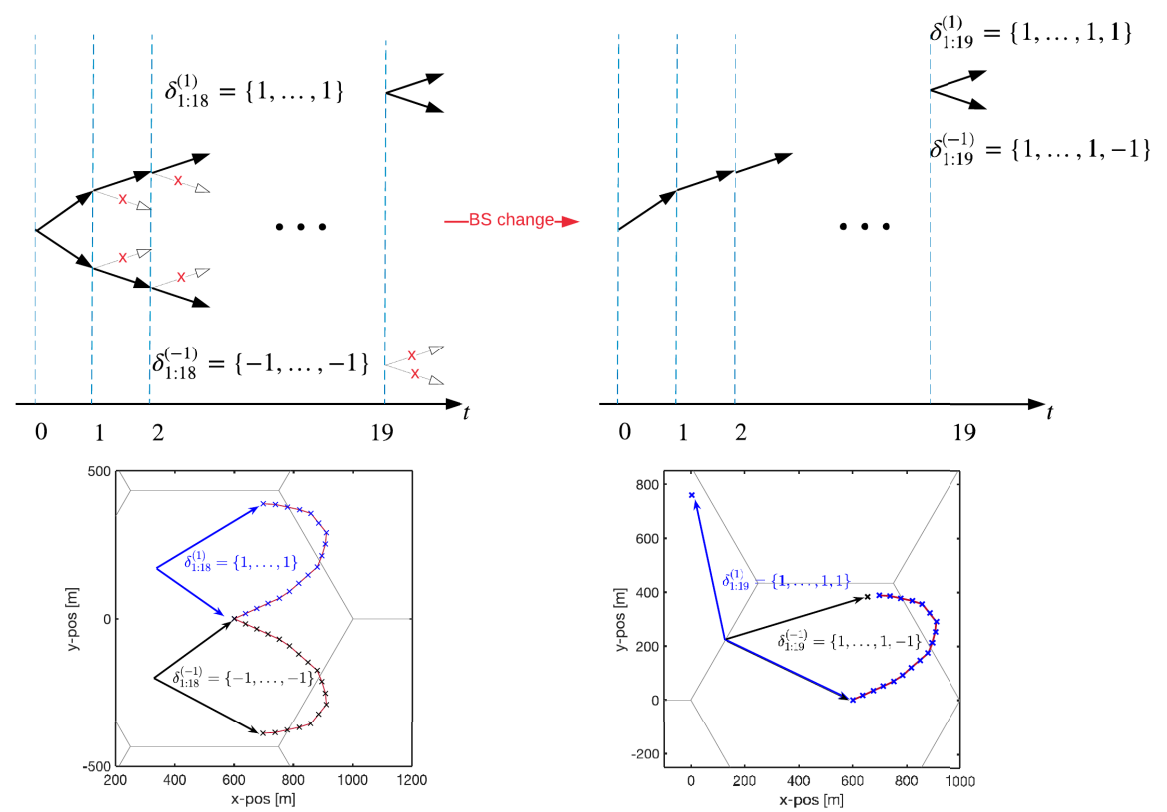

Figure 5.5: Mode sequences of the first 19 seconds corresponding to the simulated network. During the first 18 seconds, both solutions have equal weights. At $t=19$, when the set of BSs change, the wrong branch is discarded.

In order to formulate the state selection procedure for a filter bank with general $M$ pruned branches, assume that $\tilde{t}$ is the time at which the set of BSs change and $\tilde{t}_{0}$ be the last time until which state and covariance estimates are available. In the MAP sense, the state and covariance estimates are obtained by first finding the mode with highest probability at time $\tilde{t}$

$$
\hat{\delta}=\underset{\delta}{\arg \max } w_{\tilde{t}}(\delta)
$$


and then

$$
\begin{aligned}
\hat{\boldsymbol{x}}_{\tilde{t}_{0}}: \tilde{t} & =\hat{\boldsymbol{x}}_{\tilde{t}_{0}: \tilde{t}}(\hat{\delta}) \\
\hat{\boldsymbol{P}}_{t_{0}}: \tilde{t} & =\hat{\boldsymbol{P}}_{t_{0}: \tilde{t}}(\hat{\delta})
\end{aligned}
$$

Additionally, it is possible to find the minimum variance estimate for all the $M$ remaining branches based on

$$
\begin{aligned}
& \hat{\boldsymbol{x}}_{t}=\sum_{\delta_{t}} w_{t}(\delta) \hat{\boldsymbol{x}}_{t}(\delta) \\
& \hat{\boldsymbol{P}}_{t}=\sum_{\delta_{t}} w_{t}(\delta)\left[\hat{\boldsymbol{P}}_{t}(\delta)+\left(\hat{\boldsymbol{x}}_{t}(\delta)-\hat{\boldsymbol{x}}_{t}\right)\left(\hat{\boldsymbol{x}}_{t}(\delta)-\hat{\boldsymbol{x}}_{t}\right)^{T}\right]
\end{aligned}
$$

\subsubsection{Lower bounds on position estimation}

In this section, we study lower bound on the estimated positions for both snapshot and filtering estimators. Since no information of the true mode of the system is available, the Cramér-Rao Lower Bound (CRLB) analysis for the snapshot method is performed for the true mode of the system. For the dynamic case, we introduce a performance measure on the covariance of state estimates $\hat{\boldsymbol{x}}_{t \mid t}$ using measurements $y_{1: t}$.

\section{CRLB for the static case}

The theoretical lower bound on the covariance matrix of the estimation error, for any unbiased estimator, can be obtained from the CRLB theory given that certain regularity conditions are satisfied [79]. Let $\hat{\theta}(y)$ denote the estimator of the parameter vector $\theta$ from uncertain measurements $y$ in an static system. The lower bound on the covariance, $P^{\mathrm{CRLB}}$, is the inverse of the Fisher information matrix (FIM) $\boldsymbol{J}(\boldsymbol{\theta})$ [41], [42]. Given the statistical model $p(\boldsymbol{y} ; \boldsymbol{\theta})$ of the measurement vector $y$ parameterized by the deterministic unknown parameters $\theta$, the FIM is defined by

$$
[\boldsymbol{J}(\boldsymbol{\theta})]_{i, j}=\mathbb{E}\left[\frac{\partial \ln p(\boldsymbol{y} ; \boldsymbol{\theta})}{\partial \boldsymbol{\theta}^{(i)}} \frac{\partial \ln p(\boldsymbol{y} ; \boldsymbol{\theta})}{\partial \boldsymbol{\theta}^{(j)}}\right]=-\mathbb{E}\left[\frac{\partial^{2} \ln p(\boldsymbol{y} ; \boldsymbol{\theta})}{\partial \boldsymbol{\theta}^{(j)} \partial \boldsymbol{\theta}^{(j)}}\right],
$$

where the expectation is taken with respect to $p(y ; \boldsymbol{\theta})$. The covariance of $\hat{\boldsymbol{\theta}}(\boldsymbol{y})$ is bounded from below by the inverse of (5.20),

$$
\operatorname{Cov}(\hat{\theta}(y)) \triangleq \mathbb{E}_{y}\left[(\hat{\theta}(y)-\theta)(\hat{\theta}(y)-\theta)^{T}\right] \geq J(\theta)^{-1},
$$

where $\operatorname{Cov}(\hat{\boldsymbol{\theta}}(\boldsymbol{y}))-\boldsymbol{J}(\boldsymbol{\theta})^{-1} \geq \mathbf{0}$ denotes positive definiteness of the resulting matrix. The required regularity conditions under which (5.21) holds are:

1. $\frac{\partial \ln p(y ; \theta)}{\partial \theta^{(i)}}$ exists and is integrable for the whole parameter vector space

2. $\frac{\partial^{2} \ln p(y ; \theta)}{\partial \boldsymbol{\theta}^{(j)} \partial \boldsymbol{\theta}^{(j)}}$ exists and is integrable for the whole parameter vector space 
The special case of Gaussian observations of the form $y \sim \mathcal{N}(\mu(\theta), C(\theta))$, where both the mean and covariance depend on parameter vector $\theta$, is discussed for example in [72]. Assuming that the mode of the system is known a priori, the CRLB for the parameter vector $\theta=\left(\theta_{x}, \theta_{y}\right)^{T}$ is then computed from the inverse of the Gaussian FIM. Let $\boldsymbol{r}(\boldsymbol{\theta})=\left(r_{1}(\boldsymbol{\theta}), r_{21}(\boldsymbol{\theta})\right)^{T}$ be the vector function of distances, the Gaussian FIM for the special case of this problem is given by

$$
[\boldsymbol{J}(\boldsymbol{\theta})]_{i, j}=\left[\frac{\partial \boldsymbol{r}(\boldsymbol{\theta})}{\partial \theta^{(i)}}\right]^{T} \boldsymbol{R}^{-1}\left[\frac{\partial \boldsymbol{r}(\boldsymbol{\theta})}{\partial \theta^{(j)}}\right] .
$$

Figure 5.6 presents the expected lower bound on the location estimation error, assuming $\sigma=8 \mathrm{~m}$ and known mode of the system $\delta$, for the simulated network illustrated in Figure 5.3. While an error of below $80 \mathrm{~m}$ is expected in most of the area inside the BS coverage, there are certain points where the expected positioning error is extremely large. The reason is that the geometry of the two measured BSs relative to the UE that makes the positioning problem ill-posed. That is, the set of involved BSs and the UE get aligned on nearly a straight line so that the information matrix (5.22) becomes ill-conditioned.

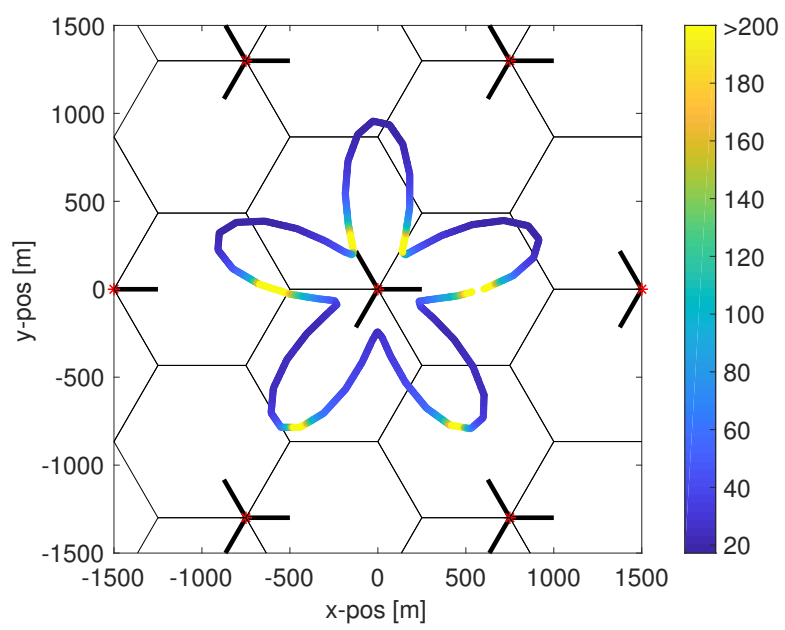

Figure 5.6: $C R L B$ of position estimation error assuming $\sigma=8 \mathrm{~m}$. The error is given for the trajectory of the simulated network illustrated in Figure 5.3. In most parts of the trajectory, marked with dark blue, low theoretical bounds on positioning errors are expected. However, there are certain parts of the trajectory, marked with yellow, large positioning errors are predicted.

\section{Approximate performance measure for the dynamic case}

The exponential growth of hypotheses of the optimal filter, from which the Mean Squared Error (MSE) can be computed, make it intractable to compute. Thus, 
we derive an approximate performance measure of the optimal filter bank for a linearized model, where each model is linearized around the true position.

The original TOF and TDOA measurement models (2.5) and (2.4) can be written as a nonlinear function $\boldsymbol{h}_{t}\left(\boldsymbol{x}_{t}\right)=\left(r_{i}, r_{j}-r_{i}\right)^{T}$ where the indices $i, j$ correspond to the serving and neighboring base stations, respectively. Linearizing around the two solutions (from the circle-hyperbola intersection problem) using a first-order Taylor series gives

$$
\begin{aligned}
\boldsymbol{x}_{t+1} & =\boldsymbol{F} \boldsymbol{x}_{t}+\omega_{t} \\
z_{t} & =\boldsymbol{H}_{t}^{\operatorname{lin}}(\delta) \boldsymbol{x}_{t}+c_{t}^{\operatorname{lin}}(\delta)+\boldsymbol{e}_{t}
\end{aligned}
$$

where $\boldsymbol{c}(\delta)$ is the linearization constant and $\boldsymbol{H}_{t}^{\text {lin }}(\delta)=\left.\frac{\partial}{\partial \boldsymbol{x}} \boldsymbol{h}_{t}\left(\boldsymbol{x}_{t}\right)\right|_{\boldsymbol{x}_{t}=\boldsymbol{\theta}^{(\delta)}}$ with $\delta \in$ $\{1,-1\}$ and where we define that $\delta=1$ assigns the true solution and $\delta=-1$ the shadow solution. The performance measure of the approximated jump Markov linear model can then be computed.

A filter bank algorithm generally does not know which mode is the correct one and thus has to estimate it together with the unknown state $\boldsymbol{x}_{t}$. What follows is an approximate measure to predict the performance of any such filter bank algorithm. Let $\delta_{1: t}^{(1)}=\{1, \ldots, 1\}$ denote the mode sequence containing the true solutions and let $\delta_{1: t}^{(-1)}=\{-1, \ldots,-1\}$ denote the mode sequence of ambiguous solutions. Further, let $\left\{\hat{\boldsymbol{x}}_{t \mid t}^{(i)}\left(z_{1: t}^{0}\right), \boldsymbol{P}_{t \mid t}^{(i)}\right\}_{i \in\{-1,1\}}$ denote the sufficient statistics of the mode conditioned posterior $p\left(x_{t} \mid \delta_{1: t}^{(i)}, z_{1: t}^{0}\right)$ of the optimal Kalman filter, where $z_{1: t}^{0}$ is the noise-free version of (5.23). Then, a performance measure is given by

$$
\boldsymbol{C}_{t \mid t}^{\mathrm{PM}}=\sum_{i \in\{-1,1\}} \alpha_{t}(i)\left[\boldsymbol{P}_{t \mid t}^{(i)}+\left(\hat{\boldsymbol{x}}_{t \mid t}^{(i)}\left(\boldsymbol{z}_{1: t}^{0}\right)-\hat{\boldsymbol{x}}_{t \mid t}\left(z_{1: t}^{0}\right)\right)\left(\hat{\boldsymbol{x}}_{t \mid t}^{(i)}\left(\boldsymbol{z}_{1: t}^{0}\right)-\hat{\boldsymbol{x}}_{t \mid t}\left(\boldsymbol{z}_{1: t}^{0}\right)\right)^{T}\right]
$$

where $\hat{\boldsymbol{x}}_{t \mid t}\left(z_{1: t}^{0}\right)=\sum_{i \in\{-1,1\}} \alpha_{t}(i) \cdot \hat{\boldsymbol{x}}_{t \mid t}^{(i)}\left(z_{1: t}^{0}\right)$ and $\alpha_{t}(i)=\operatorname{Pr}\left\{\delta_{1: t}^{(i)} \mid z_{1: t}^{0}\right\}$. Ideally, the weighting factors $\alpha_{t}$ in (5.24) are $\alpha_{t}(1)=\alpha_{t}(-1)=0.5$ whenever the branches are initiated from two solutions that are either very close to each other or are equal. In the rest of the trajectory, the tracking filter does not allow for a big jump between the current estimate and the arriving measurement resulting in zero weight for the wrong solution. Figure 5.7 illustrates ambiguities in solutions for the case of ideal mode probability weightings corresponding to the simulated network deployment whose two possible solutions are given in Figure 5.3.

\subsection{Result and conclusion}

The filter bank solution for positioning using two BSs proposed in Section 5.1 is numerically evaluated and the results are presented for both the simulated network and for the real experiments. 


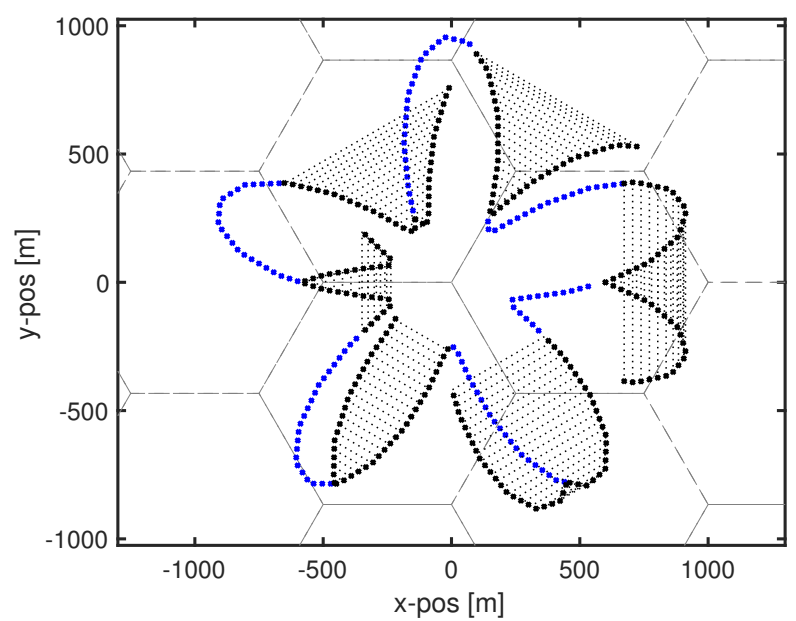

Figure 5.7: Ambiguity in position estimates with respect to possible branches over the trajectory of the simulated network illustrated in Figure 5.3. Blue dots indicate the true position of the UE. Black dots are used to indicate areas at which the second solution, marked with black, is equally likely as the first solution.

\subsubsection{Simulation study}

The simulated cellular network consists of 7 macro sites each having 3 cells, located in a hexagonal grid with an inter-site distance of $1500 \mathrm{~m}$. The UE starts at a certain point in the cellular network and passes through the network with a pre-defined flower-shape path. Figure 5.3 illustrates the cellular network deployment in which the red dots are macro sites, each with three cells, and the green line represents the true UE trajectory.

The noise-free scatter plot of two solutions obtained from the UT approximations are also given in Figure 5.3. The mode of the system changes along the trajectory depending on the set of measuring BSs and their geometrical positions. For instance, in the beginning, $\theta=[600,0]^{T}, B S 5$ and $B S 1$ are the set of measured BSs. The true position of the system thus corresponds to the mode $\delta=1$. As the UE moves along the trajectory at the position $\theta \approx[680,400]^{T}$ the measured base stations switches to BS5 and BS2. The position of the system then corresponds to the situation with mode $\delta=-1$.

In the simulations, standard deviation of the process noise is set to $\sigma_{\omega}=$ $1 \mathrm{~m} / \mathrm{s}^{2}$. The covariance matrix for the measurement noise is given by $\boldsymbol{\Lambda}_{t}(\delta)$, estimated using (5.9b). The sampling period for the simulation scenario is $T=50 \mathrm{~s}$. The positions are initialized randomly inside a circle of radius $50 \mathrm{~m}$ centered at the closest BS and the initial velocity along the $x$-and $y$-axis is set to zero, $v_{x_{0 \mid 0}}=v_{y_{0 \mid 0}}=0 \mathrm{~m} / \mathrm{s}$. The quantities for the initial uncertainty of the states, $\boldsymbol{P}_{0 \mid 0}$, are $\sigma_{x_{0}}=\sigma_{y_{0}}=10 \mathrm{~m}$ and $\sigma_{v x_{0}}=\sigma_{v y_{0}}=1 \mathrm{~m} / \mathrm{s}$. 


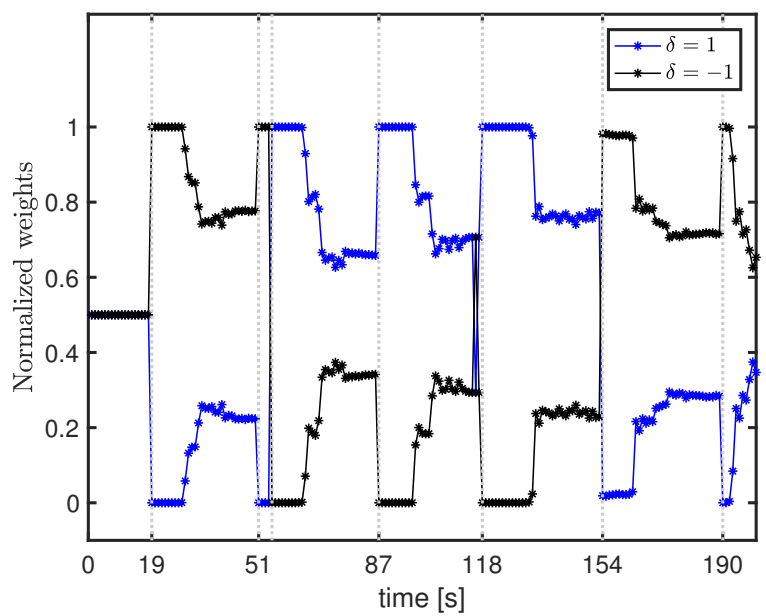

Figure 5.8: Normalized weights after the pruning stage for the filter bank with $M=2$ branches. The handover times are indicated with black dashed bars.

Figure 5.8 illustrates the Normalized weights after the pruning stage of each mode along the trajectory for one Monte Carlo run. The filter bank is allowed to have branches resulting $M=2$. The handover times are marked with the dashed lines. As soon as a handover occurs, the information obtained from the change in the set of involved BSs results in a jump in the probability of the modes. For instance, as Figure 5.8 suggests, initializing the filter bank with equal probabilities for two modes, results in equal weights until $t=19 \mathrm{~s}$. During this interval, the UE true position corresponds to the estimates with mode $\delta=1$. At $t=19 \mathrm{~s}$, when the set of involved BSs change, the second mode, marked with black, contains the UE position.

The performance of the KF bank estimator is evaluated by repeating the simulations for a total number of 1000 Monte Carlo runs. In each run, the filter bank is allowed to have $M=2$ pruned modes. The numerical RMSE is then compared to the performance measure introduced in Section 5.1.3. The values for the bound are also obtained over 1000 runs of measurement realizations.

Figure 5.9a presents the comparison between RMSE, computed for the MMSE estimate of the filtering posterior distribution of the filter bank, and the square root of $\operatorname{tr}\left(C_{t \mid t}^{\mathrm{PM}}\right)$ introduced in (5.24). Figure 5.9b, presents how the positioning error is improved by using additional information obtained from handovers. That is, the positioning error is given for the MMSE estimates $\hat{\boldsymbol{x}}_{t \mid t}$ given in (5.18b) of posterior distribution $p\left(x_{t} \mid \delta_{1: \tilde{t}, 1: t}\right)$ where $\tilde{t}>t$. Once the true mode sequence up to the time instance of BS change is known, the states are estimated. Although the estimation error for the proposed algorithm is promising, it must be noted that in the simulated network, non-line-of-sight conditions are not considered.

The scatter plot of the estimated positions of the KF bank estimator averaged 


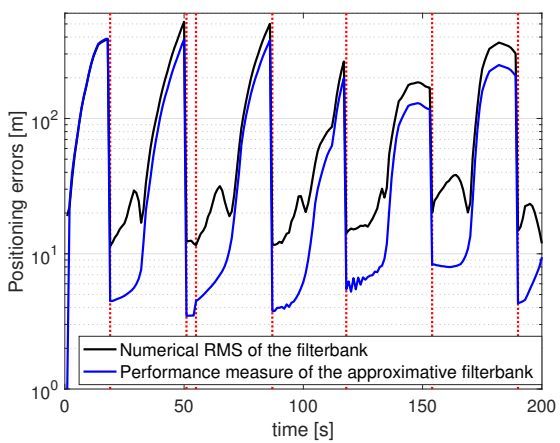

(a) Approximative filterbank performance measure.

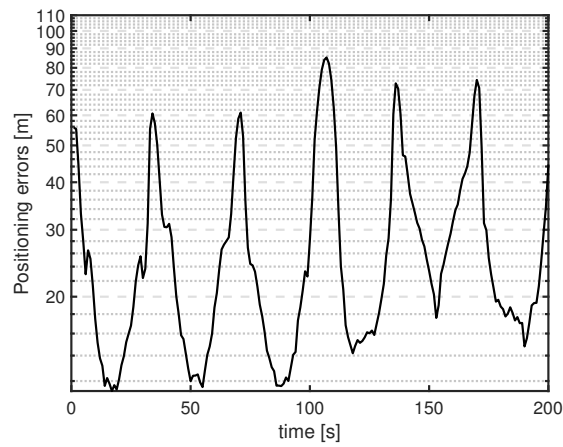

(b) Proposed filter.

Figure 5.9: Performance measure of the approximative filterbank (5.24), RMSE of the MMSE estimate $p\left(x_{t} \mid y_{1: t}\right)$, and RMSE of the MMSE estimate $p\left(\boldsymbol{x}_{t} \mid \delta_{1: \tilde{t}}, y_{1: t}\right)$ where $\tilde{t}>t$, obtained in the simulation in logarithmic scale. The handover times are indicated with red bars in Figure 5.9a.

over 1000 Monte Carlo runs is presented in Figure 5.10.

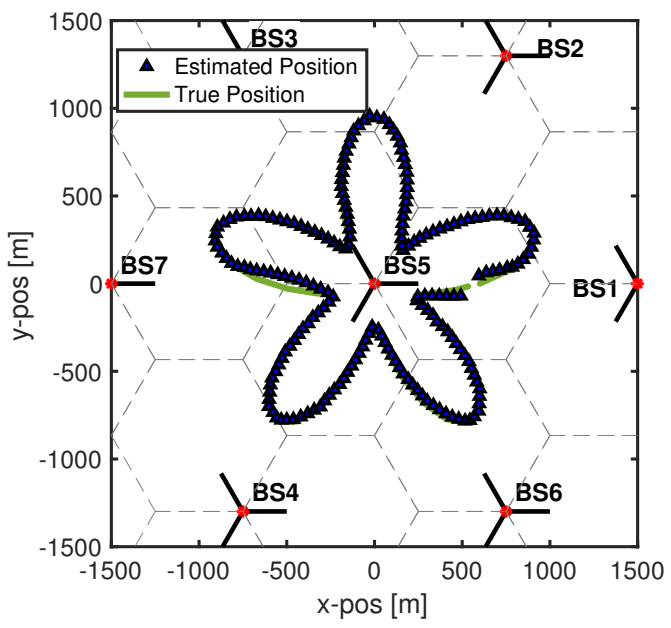

Figure 5.10: Scatter plot of estimated trajectory for the simulated network. 


\subsubsection{Experimental results}

Three separate antennas were used to collect measurements in the Kista area in Stockholm, Sweden. The network mimics a macro-cell deployment of LTE in urban areas. The inter-site distance of BSs were between $350 \mathrm{~m}$ to $600 \mathrm{~m}$ where the height of all the antennas were few meters above the average height of the buildings in the surrounding environment.

The synchronization of the transceivers were highly accurate, with standard deviation of less than $10^{-12} \mathrm{~s}$. The ground truth trajectory is obtained by logging GPS measurements. The whole trajectory took 18 minutes to be measured. For a more detailed description of hardware and the measurement campaign, see [90]. Figure 5.11 presents the measurement path, color coded based on the distance of the UE to each BS.
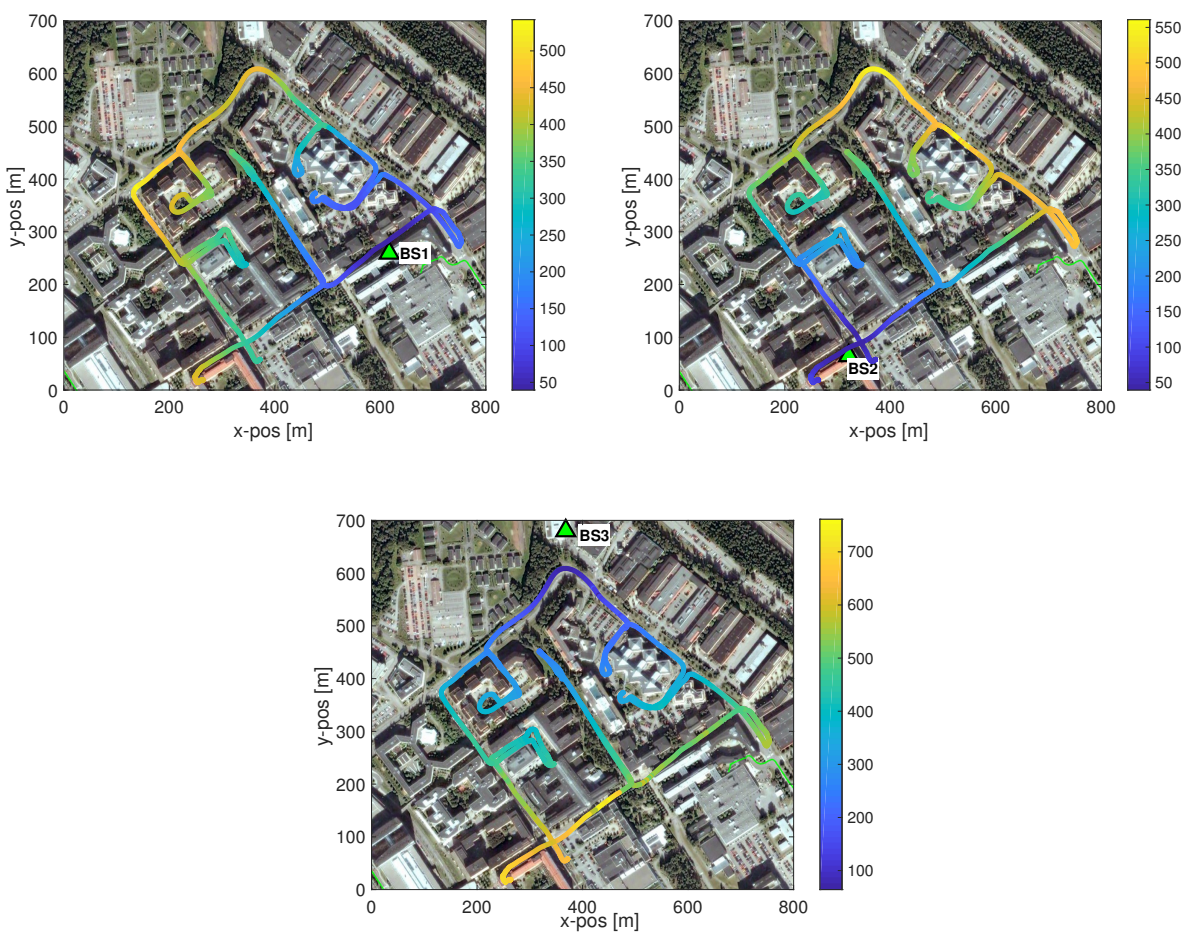

Figure 5.11: Distance of the UE from the three BSs along the whole path.

The performance of the proposed method is evaluated for the experimental scenario in terms of the scatter plot of the estimated position and the positioning error. The sampling period of the filters for the real data is $T=5 \mathrm{~s}$. The filters are initialized at the closest BSs with $v_{x_{0 \mid 0}}=v_{y_{0 \mid 0}}=0$. The quantities for the initial uncertainties are the same as the simulation scenario.

Figure 5.12 presents the estimated positions on top of the true position for 


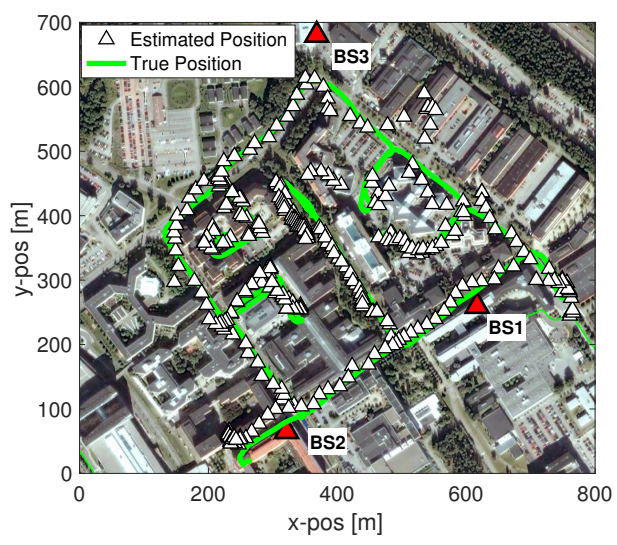

Figure 5.12: The final KF bank estimates, marked with white, on top of the GPS-logged positions of the UE, marked with green.

the whole path in the network. Most of the times, the estimated positions follow the true trajectory with high accuracy. However, in the area $x \approx[500,600]^{\mathrm{T}}$ and $y \approx[400,500]^{\mathrm{T}}$, the measurements suffer from large NLOS conditions, [90]. The poor accuracy in some areas of the real network deployment scenario is due to both the geometry of the antennas and the UE and also as a result of NLOS effects in the measurements.

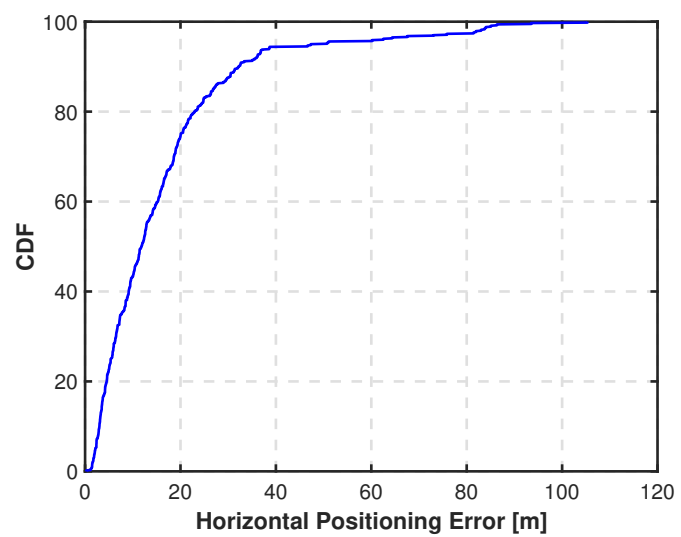

Figure 5.13: The CDF of the horizontal positioning error of the KF bank estimator applied on the data from real experiments. 


\subsubsection{Conclusions}

In this chapter, a filter bank framework was introduced for positioning based on TOF and TDOA measurement reports obtained from two base stations. We first derived the two possible analytical solutions of the intersection of TOF circle and TDOA hyperbola. Nonlinear mapping between the collected noisy measurements to the $2 \mathrm{D}$ positions of the UE was then approximated using an estimator based on the unscented transformation.

In order to estimate the UE's position from the two UT estimates, they were fed into a filter bank as pseudo measurements of the position of the UE. We note that, while the UE goes through the network, the set of involved BSs for TOF and TDOA estimations changes. The additional information obtained from the handover was then used by the filter bank to keep only the mode sequence(s) that corresponds to the UE's position and discard the rest. This allows us to deal with the ambiguity in the UE's position in cost of a certain delay.

The performance bounds of the filter bank was derived and compared to the RMSE in a simulation study. Further, the developed filtering framework was tested on real-field data collected from the network in the Kista area in Stockholm, Sweden. The results indicated good performance for simulation study and the experimental data. 


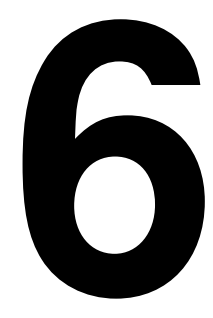

\section{Non-line-of-sight Error Estimation}

Localization error in timing-based systems highly depends on both the hardware used for timing measurements, the characteristics of the radio channel and on the geometry of BSs relative to the UE. For instance, in TOA methods, the synchronization of the BSs is one dominant factor where a synchronization error in the order of $100 \mathrm{~ns}$ causes a localization error of $30 \mathrm{~m}$. Various synchronization schemes have been studied in the literature, where GPS synchronization seems to be one of the best solutions leading to a theoretical synchronization on the order of nanoseconds.

Timestamp accuracy is another crucial factor in all timing-based methods. The timestamp of each radio signal reflect the true propagation time plus processing times at both the transmitter and the receiver. While processing time at the BSs is equal for all the involved BSs, the delay imposed by the physical and media access control (MAC) layers results in timestamp accuracies on the order of microseconds. One mitigation to the delay problem is to use a physical layer timestamp to at least avoid the delay caused by the MAC layer. This can be further improved by introducing sub-sampling steps in the physical layer, but is beyond the scope of this thesis and hence not discussed further.

Various environmental characteristics in wireless networks can also affect the localization accuracy of positioning systems in general. Timing-based systems, specifically, have two major sources of error; measurement noise and NLOS propagation errors. Measurement noise is usually modeled as a zero-mean Gaussian random variable, $\mathcal{N}\left(0, \boldsymbol{R}_{l s}\right)$, while NLOS error usually has a positive mean.

In this chapter we investigate the problem of modeling TOA errors in presence of NLOS components in terms of Gaussian mixtures. The background theory is given in Section 6.1 in which three different algorithms are introduced for Gaussian mixture parameter estimation. Finally, Section 6.2 evaluates the performance of all three algorithms and models the real data TOA error using the 
introduced quasi-Bayesian algorithm.

\subsection{Background theory}

NLOS components make the measurement errors inherently multimodal. In cases where the underlying distribution is multimodal, modeling with a single Gaussian, might lead to a mean value in region with low probability and hence overestimating the covariance. A classic example is the "Old Faithful" dataset containing measurements of the time between eruptions against duration of eruptions, both in minutes, of a geyser. Figure 6.1a presents scatter plot of the measurements together with a single Gaussian fit where the fit fails to capture the two dominant clumps of the data leading to a poor mean estimate and large covariance. Figure 6.1b, presents how a Gaussian mixture model can better represent the data. A more sophisticated algorithm is thus required to better model the

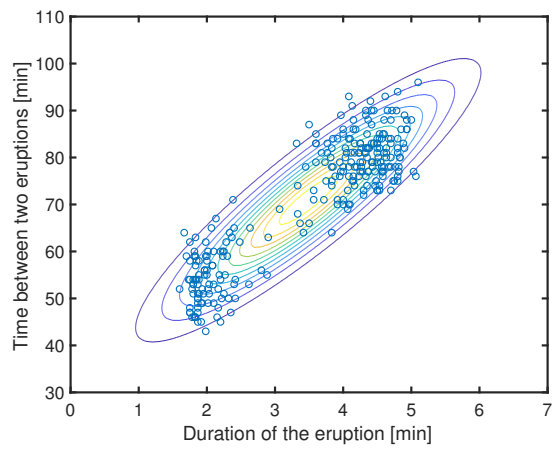

(a) Single Gaussian.

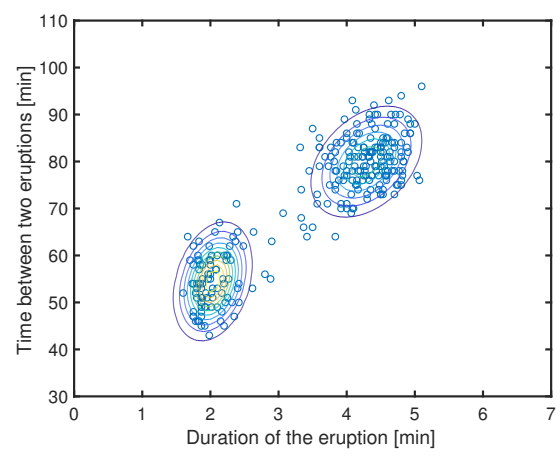

(b) Two-component Gaussian mixture.

Figure 6.1: Fitted Gaussian models to the Old Faithful dataset.

noise under NLOS conditions. In this section, we briefly introduce Gaussian mixture models followed by two well-known methods, EM and Gibbs-sampling, for estimating unknown parameters of the model. Then, a quasi-Bayes (QB) estimator is given as an alternative algorithm for joint estimation of the mixing probabilities and mixture components.

\subsubsection{Gaussian mixture models}

The multivariate Gaussian distribution of a $D$-dimensional random vector $\boldsymbol{e}$ denoted by $\mathcal{N}\left(\mu \in \mathcal{R}^{D}, \Sigma \in \mathcal{R}^{D \times D}\right)$ has the form

$$
\mathcal{N}(\boldsymbol{e} \mid \boldsymbol{\mu}, \Sigma)=\frac{1}{\left((2 \pi)^{D / 2}|\Sigma|\right)^{1 / 2}} \exp \left\{\frac{-1}{2}(\boldsymbol{e}-\boldsymbol{\mu})^{T} \Sigma^{-1}(\boldsymbol{e}-\boldsymbol{\mu})\right\}
$$


As the example in Section 6.1 suggested, a single Gaussian might fail to capture the underlying distribution of a multimodal dataset correctly. A powerful tool is to use Gaussian mixture models formed by a linear combination of basic Gaussian densities (6.1). Using a sufficient number of densities with properly chosen coefficients and adjusted means and covariances, almost all complex continuous densities can be accurately approximated [21].

Let $k \in\{1,2, \ldots, K\}$ represent the number of mixture components each defined by $\mathcal{N}\left(\boldsymbol{e} \mid \boldsymbol{\mu}^{(k)}, \Sigma^{(k)}\right)$. Further, let $\pi^{(k)}$ denote the mixing coefficients, that satisfy $0 \leq \pi^{(k)} \leq 1$ and $\sum_{k=1}^{K} \pi^{(k)}=1$. While Gaussian mixture models can be used in different applications, in this thesis we are interested in joint estimation of $\pi^{(k)}$, $\mu^{(k)}$ and $\sigma^{(k)}$ given a set of random TOA measurement errors including both measurement noise and NLOS propagation errors. Although the error terms are one dimensional, in this section we generalize our study to multi-dimensional random variables. Applying the generalized algorithms to the one-dimensional TOA errors is then straightforward. Let $\boldsymbol{e}=\left\{\boldsymbol{e}_{n}\right\}, n \in\{1, \ldots, N\}$ be a set of independent random variables collected from $N$ observations.

In order to motivate the EM method, in this thesis, we formulate Gaussian mixtures in terms of latent variable models. For this purpose, we define the discrete latent variable $s_{n} \in\{1, \ldots, K\}$ representing a discrete latent state. This way, one can interpret the mixing coefficients as prior probabilities such that $p\left(s_{n}=k\right)=\pi^{(k)}$. The joint distribution of $\boldsymbol{e}_{n}$ and $s_{n}$ is given by

$$
p\left(\boldsymbol{e}_{n}, s_{n}\right)=p\left(s_{n}\right) p\left(\boldsymbol{e}_{n} \mid s_{n}\right),
$$

with

$$
\begin{aligned}
p\left(s_{n}\right) & =\prod_{k=1}^{K}\left(\pi^{(k)}\right)^{\mathbb{I}\left(s_{n}=k\right)}, \\
p\left(\boldsymbol{e}_{n} \mid s_{n}\right) & =\prod_{k=1}^{K} \mathcal{N}\left(\boldsymbol{e}_{n} \mid \boldsymbol{\mu}^{(k)}, \Sigma^{(k)}\right)^{\mathbb{I}\left(s_{n}=k\right)},
\end{aligned}
$$

where $\mathbb{I}\left(s_{n}=k\right)$ is the indicator function. Finally, the Gaussian mixture distribution can be obtained by marginalizing $(6.2 \mathrm{a})$ over $s_{n}$ and is given by

$$
p\left(\boldsymbol{e}_{n}\right)=\sum_{s} p\left(\boldsymbol{e}_{n}, s_{n}\right)=\sum_{k=1}^{K} \pi^{(k)} \mathcal{N}\left(\boldsymbol{e}_{n} \mid \boldsymbol{\mu}^{(k)}, \Sigma^{(k)}\right) .
$$

Defining vectors $\pi=\left(\pi^{(1)}, \pi^{(2)}, \ldots, \pi^{(K)}\right), \boldsymbol{\mu}=\left(\boldsymbol{\mu}^{(1)}, \boldsymbol{\mu}^{(2)}, \ldots, \boldsymbol{\mu}^{(K)}\right)$, $\Sigma=\left(\operatorname{Sigma}^{(1)}, \boldsymbol{S i g m a} \boldsymbol{a}^{(2)}, \ldots, \boldsymbol{S i g m a} \boldsymbol{a}^{(K)}\right)$ the likelihood function of the fitted Gaussian mixture model is defined by

$$
p(\boldsymbol{e} \mid \boldsymbol{\pi}, \boldsymbol{\mu}, \Sigma)=\prod_{n=1}^{N} \sum_{k=1}^{K} \pi^{(k)} \mathcal{N}\left(\boldsymbol{e}_{n} \mid \boldsymbol{\mu}^{(k)}, \Sigma^{(k)}\right) .
$$


Extra care must be taken while estimating, or learning, the parameter vector $\psi=(\pi, \mu, \Sigma)$ as the latent variables $s_{k}$ are hidden. As shown in [95], computing a MAP estimate of the posterior distribution $p(\psi \mid \boldsymbol{e})$ is a non-convex problem. Additionally, it is shown that the parameters are not identifiable and hence $p(\psi \mid \boldsymbol{e})$ is multimodal, since a unique maximum likelihood estimator does not exist. To deal with these issues, authors in [43] employed gradient-based methods to compute the local minimum of the negative log likelihood. While this might be feasible in many cases, it imposes some constraints, see [95]. In the following, three alternatives are discussed.

\subsubsection{Expectation maximization}

The membership of data points to each individual Gaussian distribution in the mixture model (6.3) is unknown. Parameters of each distribution are also unknown. The ultimate goal of the parameter estimation is then to estimate values of the distribution parameters jointly with the mixing coefficients. The EM algorithm (first introduced in [10] and re-visited in [89] and [91]) is one of the most popular techniques for parameter estimation in all models with missing data or latent variables.

The EM algorithm iteratively alternates between two steps over the observed variables $\boldsymbol{e}$ and unobserved latent variables $s$. It must be noted that EM, in general form, can be applied to all parametric mixture models. However, in this thesis, we treat the specific case of Gaussian models for which closed-form solutions of the iterations can be derived. Additionally, EM can be employed to find either MAP estimates of the parameters or to maximize the likelihood function and for continuous or discrete latent variables. Here, we use the version tailored for maximizing the likelihood function with discrete latent variables.

The log-likelihood function to be optimized, assuming that all the parameters are known, is given by

$$
\ell(\psi)=\ln p(e, s \mid \psi) .
$$

The unknown, un-observed latent variables in (6.5), however, prevent us from computing this likelihood. That is, all the information that we have about $s$ is given by the posterior distribution of $s$ given current values the observed variables and parameters, $p(\boldsymbol{s} \mid \boldsymbol{e}, \psi)$. The EM algorithm overcomes this problem by taking the expectation of (6.5) with respect to the estimated parameters at the previous step and the observed data. Let $j$ denote the iteration number, the ex- 
pectation is given by

$$
\begin{aligned}
\mathbb{E}_{s}[\ln p(\boldsymbol{e}, \boldsymbol{s} \mid \psi)] & =\sum_{n=1}^{N} \mathbb{E}_{s}\left[\ln \left(\prod_{k=1}^{K}\left(\pi^{(k)} \mathcal{N}\left(\boldsymbol{e}_{n} \mid \boldsymbol{\mu}^{(k)}, \Sigma^{(k)}\right)\right)^{\mathbb{I}\left(s_{n}=k\right)}\right)\right] \\
& =\sum_{n=1}^{N} \sum_{k=1}^{K} \mathbb{E}\left[\mathbb{I}\left(s_{n}=k\right)\left\{\ln \pi^{(k)}+\ln \mathcal{N}\left(\boldsymbol{e}_{n} \mid \boldsymbol{\mu}^{(k)}, \Sigma^{(k)}\right)\right\}\right] \\
& =\sum_{n=1}^{N} \sum_{k=1}^{K} p\left(s_{n}=k \mid \boldsymbol{e}_{n}, \psi_{j-1}^{(k)}\right) \ln \left(\pi_{k} \mathcal{N}\left(\boldsymbol{e}_{n} \mid \psi^{(k)}\right)\right) .
\end{aligned}
$$

In the $E$ step, the algorithm parameterizes the expectation (6.6a) in terms of some general $\psi$ using the current value of parameters $\psi_{j-1}$. To do so, conditional probability of $s_{n}$ given $\boldsymbol{e}_{n}$ and the best guess of parameters at iteration $j-1$, $p\left(s_{n}=k \mid \boldsymbol{e}_{n}, \psi_{j-1}\right)$ in (6.6c) should be computed. Using Bayes' theorem, this value is given by

$$
\begin{aligned}
p\left(s_{n}=k \mid \boldsymbol{e}_{n}, \psi_{j-1}^{(k)}\right) & =\frac{p\left(s_{n}=k \mid \psi_{j-1}^{(k)}\right) p\left(\boldsymbol{e}_{n} \mid s_{n}=k, \psi_{j-1}^{(k)}\right)}{\sum_{k^{\prime}=1}^{K} p\left(s_{n}=k^{\prime} \mid \psi_{j-1}\right) p\left(\boldsymbol{e}_{n} \mid s_{n}=k^{\prime}, \psi_{j-1}^{\left(k^{\prime}\right)}\right)} \\
& =\frac{\pi^{(k)} \mathcal{N}\left(\boldsymbol{e}_{n} \mid \boldsymbol{\mu}_{j-1}^{(k)}, \Sigma_{j-1}^{(k)}\right)}{\sum_{k^{\prime}=1}^{K} \pi^{\left(k^{\prime}\right)} \mathcal{N}\left(\boldsymbol{e}_{n} \mid \mu_{j-1}^{\left(k^{\prime}\right)}, \Sigma_{j-1}^{\left(k^{\prime}\right)}\right)} .
\end{aligned}
$$

The parameters are then updated in the $M$ step by maximizing the parameterized function with respect to $\psi$. This can be achieved by setting the derivative of the $\log$ of the likelihood function (6.4) with respect to $\mu^{(k)}, \Sigma^{(k)}$, and $\pi^{(k)}$ to zero individually. The closed form solutions are provided in Algorithm 5 that also summarizes iterations in the EM. It must be noted that the log-likelihood function monotonically increases in each iteration until it reaches a local optima, see [95] for proof. Thus the difference between two consecutive log-likelihood values in Algorithm 5 should always be either positive or zero.

\subsubsection{Gibbs sampling}

The frequentist EM algorithm, as stated, is a well-studied method for estimation of parameters of mixture densities together with their corresponding mixing coefficients. The algorithm is specifically powerful in the sense that it can be applied to almost any parameterized densities with any number of clusters $K$. However, as a likelihood estimator, it might get to a local maximum. Furthermore, in some applications, in addition to the point estimates given by EM, the uncertainty of the estimated values might also be of interest. In these cases, Bayesian inference approaches can be used to overcome these issues. There is a vast literature on using Bayesian inference for mixture models parameter estimation, see for instance [35], [38], [102], and [20]. Although Markov chain Monte Carlo (MCMC) 


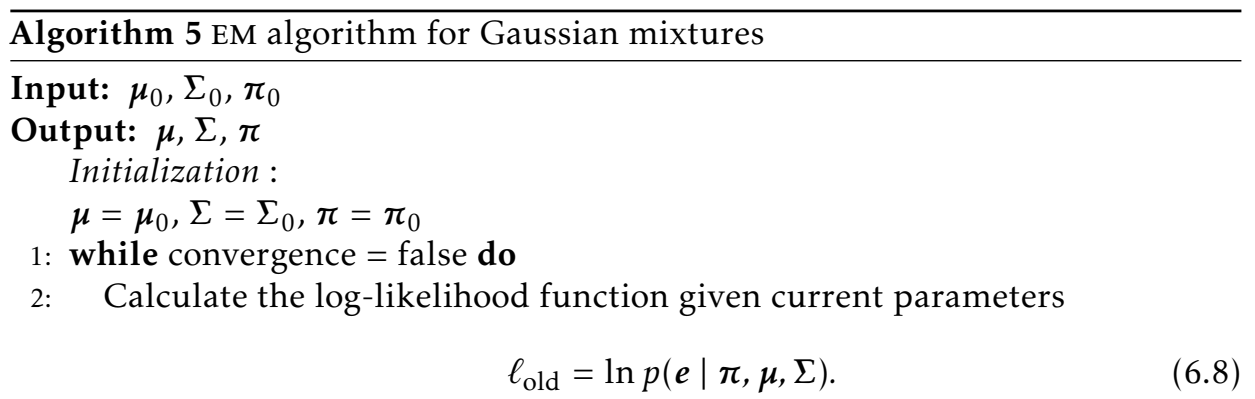

3: Perform the $E$ step by computing responsibilities for the known number of components $k=1, \ldots, K$ and for $n=1, \ldots, N$

$$
\zeta_{n k}=\frac{\pi_{K} \mathcal{N}\left(\boldsymbol{e}_{n} \mid \psi_{j-1}^{(k)}\right)}{\sum_{k^{\prime}=1}^{K} \pi_{k^{\prime}} \mathcal{N}\left(\boldsymbol{e}_{n} \mid \psi_{j-1}^{\left(k^{\prime}\right)}\right)} .
$$

4: $\quad$ Perform the $M$ step by updating the parameters for $k=1, \ldots, K$

$$
\begin{aligned}
\zeta_{k} & \triangleq \sum_{n=1}^{N} \zeta_{n k}, \\
\pi_{k} & =\frac{\zeta_{k}}{N}, \\
\boldsymbol{\mu}_{k} & =\frac{1}{\zeta_{k}} \sum_{n=1}^{N} \zeta_{n k} \boldsymbol{e}_{n}, \\
\Sigma_{k} & =\frac{\sum_{n=1}^{N} \zeta_{n k} \boldsymbol{e}_{n} \boldsymbol{e}_{n}^{T}}{\zeta_{k}}-\boldsymbol{\mu}_{k} \boldsymbol{\mu}_{k}^{T} .
\end{aligned}
$$

5: Re-calculate the log-likelihood value given the updated parameters

$$
\ell_{\text {new }}=\ln p(e \mid \pi, \mu, \Sigma) \text {. }
$$

6: Check for convergence of the algorithm. If $\ell_{\text {new }}-\ell_{\text {old }}$ is below a pre-defined threshold set convergence to true.

7: end while

8: return $\mu, \Sigma, \pi$.

methods might take more time to converge, they will reach the target distribution eventually.

One of the simplest, yet widely applicable, MCMC algorithms is Gibbs sam- 
pling, first discussed in [49]. The idea with Gibbs sampling is to draw samples of each variable conditioned on the values of all other variables. Thus, this method is known to give dependent draws of the target distribution where parameters are autocorrelated. That is why, the convergence rate might not be as fast as other techniques, for instance, assume that the goal is to draw a sample from the joint distribution of $M$ random variables, $p(\tilde{z})=p\left(\tilde{z}^{(1)}, \tilde{z}^{(2)}, \ldots, \tilde{z}^{(M)}\right)$. Given an initial sample $\tilde{z}_{0}$, we can obtain $\tilde{z}_{1}$ by drawing $\tilde{z}_{1}^{(i)}$ iteratively as follows

- $\operatorname{draw} \tilde{z}_{1}^{(1)} \sim p\left(\tilde{z}^{(1)} \mid \tilde{z}_{0}^{(2)}, \tilde{z}_{0}^{(3)}, \ldots, \tilde{z}_{0}^{(M)}\right)$

- $\operatorname{draw} \tilde{z}_{1}^{(2)} \sim p\left(\tilde{z}^{(2)} \mid \tilde{z}_{0}^{(1)}, \tilde{z}_{0}^{(3)}, \ldots, \tilde{z}_{0}^{(M)}\right)$

- $\operatorname{draw} \tilde{z}_{1}^{(M)} \sim p\left(\tilde{z}^{(M)} \mid \tilde{z}_{0}^{(1)}, \tilde{z}_{0}^{(2)}, \ldots, \tilde{z}_{0}^{(M-1)}\right)$

Repeating this procedure, the draws will eventually be made from the target distribution. It must be noted that these updates can either be performed in order or randomly. Applying Gibbs sampling in fitting mixture models to the data becomes simpler if conjugate priors are used for the unknown parameters $\psi$. We first introduce semi-conjugate priors for unknown parameters $\pi, \mu$, and $\Sigma$ of the Gaussian mixture model, for more details see [78].

\section{Prior distributions}

In the special case that each component in the mixture model has univariate Gaussian distribution, the inverse chi-squared, $\chi_{1}^{2}$ distribution can be used as a conjugate prior for the variances. For a general Gaussian mixture model with multivariate Gaussian densities, the inverse Wishart distribution, denoted by IW, is a conjugate prior for $\Sigma^{(k)}$

$$
\Sigma^{(k)} \sim \operatorname{IW}\left(\Sigma^{(k)} \mid v_{0}^{(k)}, \mathbf{\Omega}_{0}^{(k)}\right),
$$

where $v_{0}^{(k)}$ is the the degrees of freedom used to adjust the strength of the prior belief for each component and $\boldsymbol{\Omega}_{0}^{(k)}$ is the scaling matrix used to model the larger variance of a component $i$ by increasing $\Omega_{0}^{(i)}$. Subscript 0 denotes that these are prior parameters. The prior model for $\boldsymbol{\mu}^{(k)}$ is a normal distribution conditioned on the covariance matrix $\Sigma^{(k)}$

$$
\boldsymbol{\mu}^{(k)} \mid \Sigma^{(k)} \sim \mathcal{N}\left(\boldsymbol{\mu}^{(k)} \mid \boldsymbol{m}_{0}^{(k)}, \boldsymbol{V}_{0}^{(k)}\right),
$$

where $\boldsymbol{m}_{0}^{(k)}$ is the prior belief of each component's mean and $\boldsymbol{V}_{0}^{(k)}$ is defined by an additional precision parameter $\tilde{\tau}^{(k)}, V_{0}^{(k)}=\Sigma^{(k)} / \tilde{\tau}^{(k)}$. Finally, the prior distribution of mixing coefficients is given by the Dirichlet distribution with prior parameters $\alpha_{0}$

$$
\pi^{(k)} \sim \operatorname{Dir}\left(\pi \mid \alpha_{0}\right)=\frac{\Gamma\left(\alpha_{0}^{(1)}+\ldots+\alpha_{0}^{(k)}\right)}{\Gamma\left(\alpha_{0}^{(1)}\right) \times \ldots \times \Gamma\left(\alpha_{0}^{(k)}\right)}\left(\pi^{(1)}\right)^{\alpha_{0}^{(1)}-1} \times \ldots \times\left(\pi^{(k)}\right)^{\alpha_{0}^{(k)}-1} .
$$


The joint conjugate prior distribution is thus given by

$$
p(\psi)=\operatorname{Dir}\left(\boldsymbol{\pi} \mid \alpha_{0}\right) \prod_{k=1}^{K} \mathcal{N}\left(\boldsymbol{\mu}^{(k)} \mid \boldsymbol{m}_{0}^{(k)}, \boldsymbol{V}_{0}^{(k)}\right) \operatorname{IW}\left(\Sigma^{(k)} \mid v_{0}^{(k)}, \mathbf{\Omega}_{0}^{(k)}\right) .
$$

The full joint distribution is then defined by

$$
\begin{aligned}
p(\boldsymbol{e}, \boldsymbol{s}, \boldsymbol{\mu}, \Sigma, \boldsymbol{\pi}) & =p(\boldsymbol{e} \mid \boldsymbol{s}, \boldsymbol{\mu}, \Sigma) p(\boldsymbol{s} \mid \boldsymbol{\pi}) p(\boldsymbol{\pi}) \prod_{k=1}^{K} p\left(\boldsymbol{\mu}^{(k)}\right) p\left(\Sigma^{(k)}\right) \\
& =\prod_{n=1}^{N} \prod_{k=1}^{K}\left(\pi^{(k)} \mathcal{N}\left(\boldsymbol{e}_{n} \mid \boldsymbol{\mu}^{(k)}, \Sigma^{(k)}\right)\right)^{\mathbb{I}\left(s_{n}=k\right)} \times p(\psi) .
\end{aligned}
$$

\section{Posterior distribution}

The conditional posterior distributions of the latent variables and the unknown parameters can be derived from the conjugate prior distributions.

- Latent variables

$$
p\left(s_{n}=k \mid \boldsymbol{e}_{n}, \boldsymbol{\mu}, \Sigma, \boldsymbol{\pi}\right)=\frac{\pi^{(k)} \mathcal{N}\left(\boldsymbol{e}_{n} \mid \boldsymbol{\mu}^{(k)}, \Sigma^{(k)}\right)}{\sum_{k^{\prime}=1}^{K} \pi^{\left(k^{\prime}\right)} \mathcal{N}\left(\boldsymbol{e}_{n} \mid \boldsymbol{\mu}^{\left(k^{\prime}\right)}, \Sigma^{\left(k^{\prime}\right)}\right)} .
$$

- Covariance matrices

$$
p\left(\Sigma^{(k)} \mid \boldsymbol{e}, \boldsymbol{s}\right)=\operatorname{IW}\left(\Sigma^{(k)} \mid v_{0}^{(k)}+N^{(k)}, \mathbf{\Omega}_{0}^{(k)}+O^{(k)}\right),
$$

where $N^{(k)} \triangleq \sum_{n=1}^{N} \mathbb{I}\left(s_{n}=k\right)$ is the number of observations corresponding to component $k$ and

$$
\begin{aligned}
O^{(k)}= & \frac{N^{(k)} \tilde{\tau}^{(k)}}{N^{(k)}+\tilde{\boldsymbol{\tau}}^{(k)}}\left(\boldsymbol{m}_{0}^{(k)}-\overline{\boldsymbol{e}}^{(k)}\right)\left(\boldsymbol{m}_{0}^{(k)}-\overline{\boldsymbol{e}}^{(k)}\right)^{T} \\
& +\sum_{n=1}^{N} \mathbb{I}\left(s_{n}=k\right)\left(\boldsymbol{e}_{n}-\overline{\boldsymbol{e}}^{(k)}\right)\left(\boldsymbol{e}_{n}-\overline{\boldsymbol{e}}^{(k)}\right)^{T},
\end{aligned}
$$

where $\overline{\boldsymbol{e}}$ are the mean values estimated from data.

- Means

$$
p\left(\boldsymbol{\mu}^{(k)} \mid \boldsymbol{e}, \boldsymbol{s}, \Sigma^{(k)}\right)=\mathcal{N}\left(\boldsymbol{\mu}^{(k)} \mid \boldsymbol{m}^{(k)}, \boldsymbol{V}^{(k)}\right)
$$

with

$$
\begin{aligned}
& \boldsymbol{m}^{(k)}=\frac{\tilde{\boldsymbol{\tau}}^{(k)} \boldsymbol{m}_{0}^{(k)}+N^{(k)} \overline{\boldsymbol{e}}^{(k)}}{N^{(k)}+\tilde{\tau}^{(k)}} \\
& \boldsymbol{V}^{(k)}=\frac{1}{N^{(k)}+\tilde{\tau}^{(k)}} \Sigma^{(k)}
\end{aligned}
$$


- Mixing coefficients

$$
p\left(\pi^{(1)}, \ldots, \pi^{(K)} \mid s\right)=\operatorname{Dir}\left(\alpha_{0}^{(1)}+N^{(1)}, \ldots, \alpha_{0}^{(k)}+N^{(K)}\right)
$$

The Gibbs sampling method thus can be used to compute a point estimate of the unknown parameters from the posterior distributions given above. Algorithm 6 provides the $I$ iterations in a Gibbs sampler used to infer Gaussian mixture mode parameters.

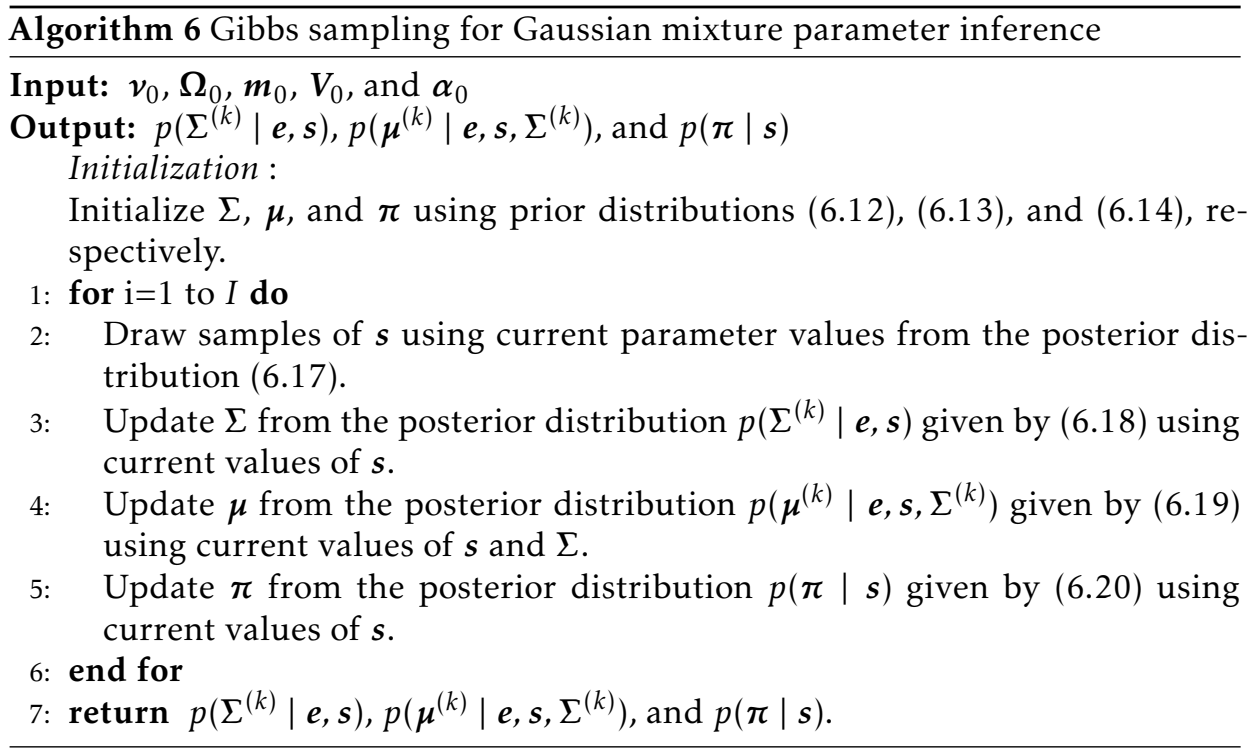

\subsubsection{Quasi-Bayes approximation}

The Bayesian algorithm for learning $\psi$ involves the specification of an a priori density for $\psi$ and the subsequent recursive computation of the posterior $p(\psi \mid \boldsymbol{e})$ as seen in the Gibbs sampling method in Section 6.1.3. This results in an unavoidable increase in computer time and memory requirements that might make the formal Bayes learning procedure less practical in some applications. As an alternative, a QB procedure which is both highly computationally efficient and retains the flavor of the formal Bayes solution, has been proposed by [86], [85], and [113]. By a suitable approximation, a recursive procedure can be obtained that can be efficiently computed.

Maximizing the observed likelihood given by

$$
p\left(\boldsymbol{e}_{1}, \boldsymbol{e}_{2}, \ldots, \boldsymbol{e}_{N} \mid \psi\right)=\prod_{n=1}^{N} p\left(\boldsymbol{e}_{n} \mid \psi\right)
$$


has the drawback of singularities in points where one of the components has a mean exactly equal to the observation with no variance, see [95]. At any such points, the likelihood value goes to infinity. The author in [56] suggests a new optimization function that includes the maximum likelihood estimator as a special case. Let the prior distributions be given by

$$
\begin{gathered}
\boldsymbol{\mu}^{(k)} \mid \Sigma^{(k)} \sim \mathcal{N}\left(\boldsymbol{m}_{0}^{(k)}, \Sigma^{(k)} / \tilde{\tau}^{(k)}\right) \\
\left(\Sigma^{(k)}\right)^{-1} \sim \operatorname{Wi}\left(\nu_{0}^{(k)}, \mathbf{\Omega}_{0}^{(k)}\right)
\end{gathered}
$$

where $\operatorname{Wi}\left(v_{0}, \boldsymbol{\Omega}\right)$ denotes the Wishart distribution. The suggested log- likelihood function is given by

$$
\begin{aligned}
\ell^{\mathrm{QB}}(\psi)= & \log p\left(\boldsymbol{e}_{1}, \boldsymbol{e}_{2}, \ldots, \boldsymbol{e}_{N} \mid \psi\right)+\sum_{k=1}^{K}\left(a^{(k)} / 2\right) \log \left|\left(\Sigma^{(k)}\right)^{-1}\right| \\
& -1 / 2 \sum_{k=1}^{K} \operatorname{tr}\left[\left(c^{(k)}\left(\boldsymbol{m}^{(k)}-\boldsymbol{\mu}^{(k)}\right)\left(\boldsymbol{m}^{(k)}-\boldsymbol{\mu}^{(k)}\right)^{T}+\boldsymbol{B}^{(k)}\right)\left(\Sigma^{(k)}\right)^{-1}\right] .
\end{aligned}
$$

Setting the design parameters $a^{(k)}=c^{(k)}=\boldsymbol{B}^{(k)}=0$ gives the maximum likelihood estimator as a special case. Instead, as shown in [56], setting $a^{(k)}=2 v_{0}^{(k)}-1$, $c^{(k)}=\tilde{\tau}^{(k)}$, and $\boldsymbol{B}^{(k)}=2 \boldsymbol{\Omega}_{0}^{(k)}$ results in the posterior mode estimator. Another interpretation of (6.23) is to see it as a penalized maximum likelihood. In this interpretation, assuming that $a^{(k)}$ and $c^{(k)}$ are greater than zero and $\boldsymbol{B}^{(k)}$ is positive definite, $\ell^{\mathrm{QB}} \rightarrow-\infty$ if $\Sigma^{(k)} \rightarrow \mathbf{0}$. The $\mathrm{QB}$ algorithm sets the design parameters based on the values of prior beliefs. That is, $a^{(k)}=v_{0}^{(k)}-K, c^{(k)}=\tilde{\tau}^{(k)}$ and $\boldsymbol{B}^{(k)}=\mathbf{\Omega}_{0}^{(k)}$.

The unknown parameters $\psi$ can be estimated from (6.23) using any numerical optimization method. However, in this thesis, we note that priors in (6.22) are independent of the latent variables. Thus, the problem fits into an EM algorithm where each parameter can be estimated in an iterative manner [10]. To do so, we set the derivative of $\ell^{\mathrm{QB}}(\psi)$ with respect to the mixture parameters to zero and then iterate in an EM algorithm by updating one parameter and fixing the others.

The $E$ step of the algorithm is similar to Section 6.1 .2 where the responsibilities are computed by

$$
\zeta_{n k}=p\left(s_{n}=k \mid \boldsymbol{e}_{n}, \psi_{j-1}^{(k)}\right)=\frac{\pi_{j-1}^{(k)} \mathcal{N}\left(\boldsymbol{e}_{n} \mid \boldsymbol{\mu}_{j-1}^{(k)}, \Sigma_{j-1}^{(k)}\right)}{\sum_{k^{\prime}=1}^{K} \pi_{j-1}^{\left(k^{\prime}\right)} \mathcal{N}\left(\boldsymbol{e}_{n} \mid \boldsymbol{\mu}_{j-1}^{\left(k^{\prime}\right)}, \Sigma_{j-1}^{\left(k^{\prime}\right)}\right)}
$$

In the $M$ step we first derive the expression for $\pi^{(k)}$ by setting the derivative of $\ell^{\mathrm{QB}}(\psi)$ with respect to $\pi^{(k)}$ to zero

$$
0=\frac{\partial \ell^{\mathrm{QB}}(\psi)}{\partial \pi^{(k)}}=\frac{\partial p\left(\boldsymbol{e}_{1}, \boldsymbol{e}_{2}, \ldots, \boldsymbol{e}_{N} \mid \psi\right)}{\partial \pi^{(k)}}
$$


Since the extra terms of $(6.23)$ do not depend on mixing coefficients, this term is also similar to Section 6.1.2, hence

$$
\pi^{(k)}=\frac{\sum_{n=1}^{N} \zeta_{n k}}{N}
$$

Then, we maximize $\boldsymbol{\mu}^{(k)}$ by setting $\frac{\partial \ell^{\mathrm{QB}}(\psi)}{\partial \boldsymbol{\mu}^{(k)}}$ to zero

$$
0=c_{k}\left(\Sigma^{(k)}\right)^{-1}\left(\boldsymbol{m}_{0}^{(k)}-\boldsymbol{\mu}^{(k)}\right)+\sum_{n=1}^{N}\left(\Sigma^{(k)}\right)^{-1}\left(\boldsymbol{e}_{n}-\boldsymbol{\mu}^{(k)}\right) \zeta_{n k}
$$

That yields

$$
\boldsymbol{\mu}^{(k)}=\frac{c^{(k)} \boldsymbol{m}_{0}^{(k)}+\sum_{n=1}^{N} \boldsymbol{e}_{n} \zeta_{n k}}{c_{k}+\sum_{n=1}^{N} \zeta_{n k}} .
$$

Finally, covariances are maximized by setting $\frac{\partial \ell^{\mathrm{QB}}(\psi)}{\partial\left(\Sigma^{(k)}\right)^{-1}}=0$

$$
\begin{aligned}
0= & 1 / 2\left(a^{(k)} \Sigma^{(k)}-c^{(k)}\left(\boldsymbol{m}_{0}^{(k)}-\boldsymbol{\mu}^{(k)}\right)\left(\boldsymbol{m}_{0}^{(k)}-\boldsymbol{\mu}^{(k)}\right)^{T}-\boldsymbol{B}^{(k)}\right) \\
& +1 / 2 \sum_{n+1}^{N}\left(\Sigma^{(k)}-\left(\boldsymbol{e}_{n}-\boldsymbol{\mu}^{(k)}\right)\left(\boldsymbol{e}_{n}-\boldsymbol{\mu}^{(k)}\right)^{T}\right) \zeta_{n k}
\end{aligned}
$$

That gives

$$
\begin{aligned}
\left(\Sigma^{(k)}\right)^{-1} & =\left(a^{(k)}+\sum_{n=1}^{N} \zeta_{n k}\right) \\
& \times\left(\boldsymbol{B}^{(k)}+\sum_{n=1}^{N} \zeta_{n k}\left(\boldsymbol{e}_{n}-\boldsymbol{\mu}^{(k)}\right)\left(\boldsymbol{e}_{n}-\boldsymbol{\mu}^{(k)}\right)^{T}+c^{(k)}\left(\boldsymbol{m}_{0}^{(k)}-\boldsymbol{\mu}^{(k)}\right)\left(\boldsymbol{m}_{0}^{(k)}-\boldsymbol{\mu}^{(k)}\right)^{T}\right)^{-1}
\end{aligned}
$$

Algorithm 7 summarizes the iterations of the QB method for learning parameters of Gaussian mixtures. The design parameters $\boldsymbol{a}^{(k)}, c^{(k)}, \boldsymbol{B}^{(k)}$, and $\boldsymbol{m}_{0}^{(k)}$ are used to initialize the parameters using prior distributions given in (6.22). The likelihood given initial parameters can then be calculated as in the E step of EM but with a different likelihood function (6.23) . The $M$ step is then performed using closedform equations $(6.26),(6.27 \mathrm{~b})$, and $(6.28 \mathrm{~b})$. The procedure is repeated until a convergence criterion is met or the maximum number of iterations is reached.

\subsection{Performance evaluation}

In this section, the performance of the three estimators are evaluated over both simulated samples and real TOA measurements collected from LTE BSs with network parameters given in Section 5.2.2. In the simulation study, three different 


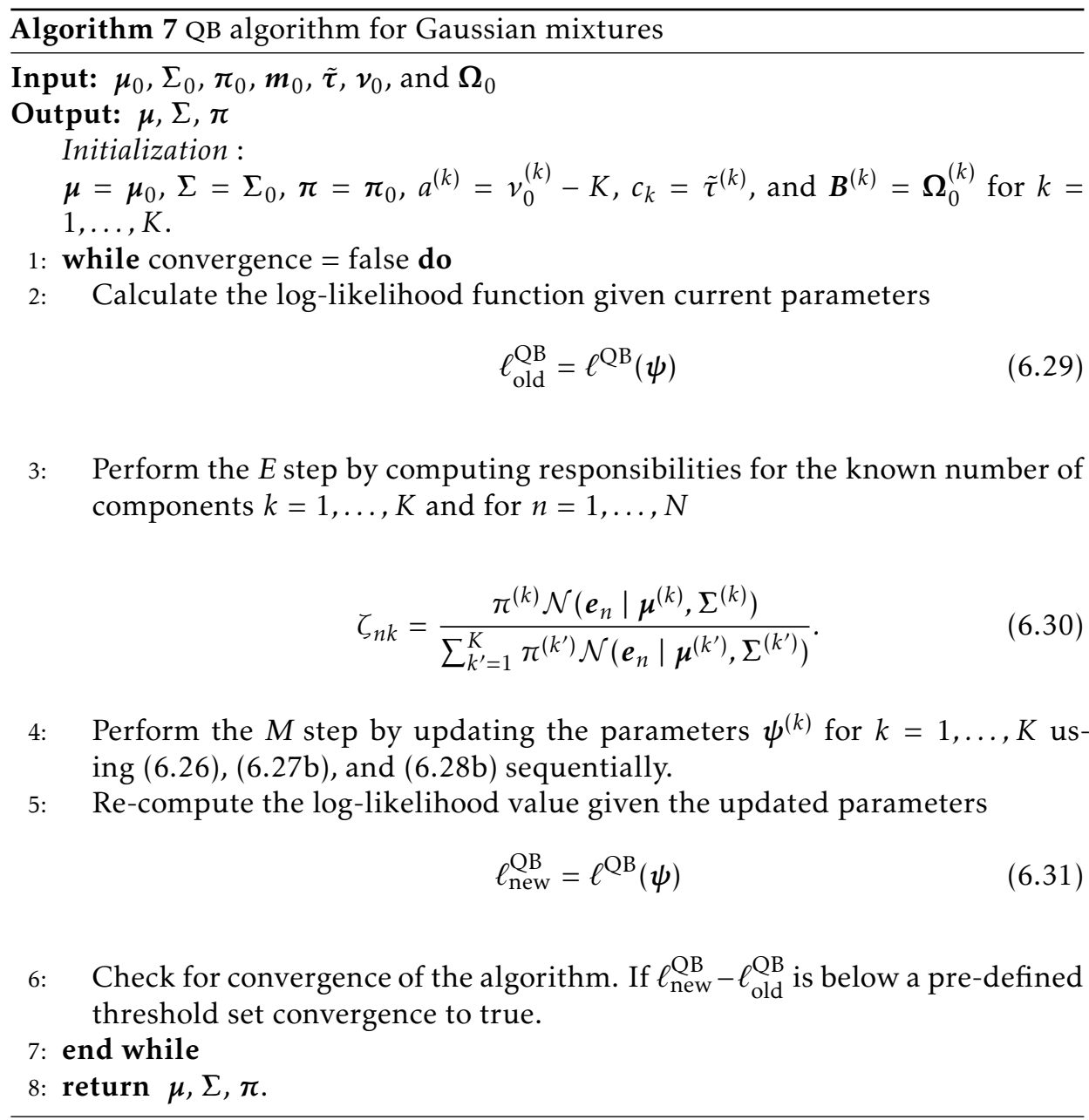

scenarios are defined. The data points in each scenario have unique characteristics in terms of their actual underlying distribution. Evaluations are performed in terms of the MSE of the estimated parameter vector given by $\sum_{j=1}^{J}\left(\hat{\psi}_{j}-\psi_{j}\right)^{2}$.

\subsubsection{Simulated model}

In all simulations, the whole dataset consists of 500 Monte Carlo samples of size $N=1000$ for a 2-component mixture of 2-dimensional Gaussian distributed random vectors. The number of unknown parameters to be estimated can be expressed as a function of components $k$ as

$$
J(k)=\frac{k\left(d^{2}+3 d\right)}{2}+k-1 .
$$


That is, in total, 11 unknown parameters should be estimated. In the first scenario, the mean of the two components are well separated from each other while covariances are equal and measurements are equally drawn from each component. In the second scenario, measurement errors in presence of outliers are simulated. For this purpose, 1 out of 100 measurements are simulated such that they come from a distribution with 4 times bigger standard deviations. The last scenario investigates the performance of each algorithm modeling the samples if the data is in fact coming from a 2-dimensional single Gaussian distribution rather than a real mixture. Figure 6.2 visualizes 1 sample of all scenarios of the simulated models and Table 6.1 summarizes the scenarios and gives their true parameters. Parameter values of the EM algorithm are initialized randomly. $\mu_{0}$

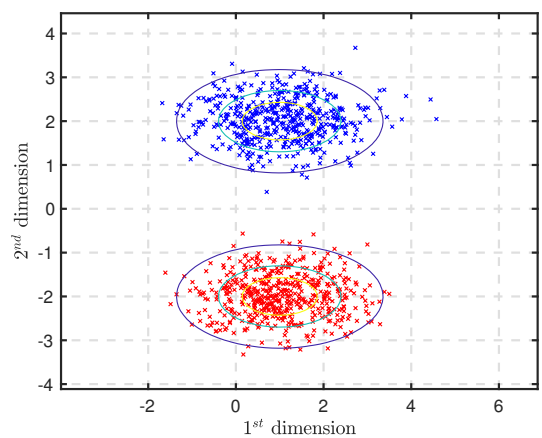

(a) Scenario 1 .

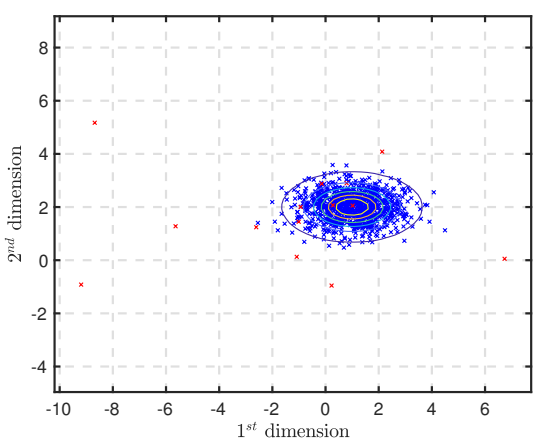

(b) Scenario 2.

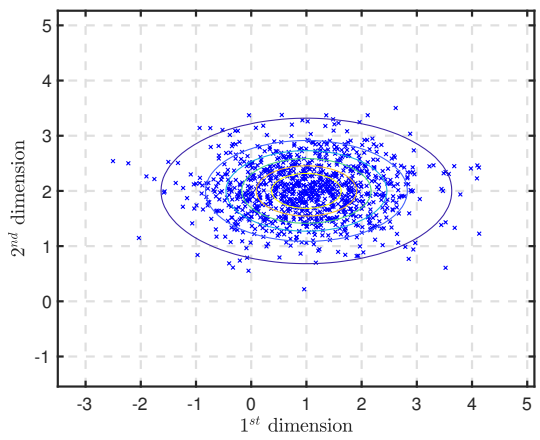

(c) Scenario 3 .

Figure 6.2: One sample of all three scenarios whose true parameters are given in Table 6.1. The first component is marked with blue and the second component with red.

in Algorithm 5 is obtained by multiplying the largest data point in each sample by a uniform distribution and $\Sigma_{0}$ is obtained by multiplying the absolute value of the largest number by an identity matrix of appropriate size. In both Gibbs 
Table 6.1: The simulated two-component mixture of two-dimensional Gaussians in three different scenarios.

\begin{tabular}{|c|c|c|c|}
\hline Scenario & mean & covariance & $\begin{array}{c}\text { mixing } \\
\text { coefficient }\end{array}$ \\
\hline \multirow[t]{2}{*}{1} & $\boldsymbol{\mu}^{(1)}=\left(\begin{array}{l}1 \\
2\end{array}\right.$ & $\Sigma^{(1)}=\left(\begin{array}{cc}1 & 0 \\
& 0.25\end{array}\right)$ & $\pi^{(1)}=0.5$ \\
\hline & $\boldsymbol{\mu}^{(2)}=\left(\begin{array}{c}1 \\
-2\end{array}\right.$ & $\Sigma^{(2)}=\left(\begin{array}{cc}1 & 0 \\
& 0.25\end{array}\right)$ & \\
\hline \multirow[t]{2}{*}{2} & $\boldsymbol{\mu}^{(1)}=\left(\begin{array}{l}1 \\
2\end{array}\right.$ & $\Sigma^{(1)}=\left(\begin{array}{cc}1 & 0 \\
& 0.25\end{array}\right)$ & $\pi^{(1)}=0.99$ \\
\hline & $\mu^{(2)}=\left(\begin{array}{l}1 \\
2\end{array}\right.$ & $\Sigma^{(2)}=\left(\begin{array}{ll}16 & 0 \\
& 4\end{array}\right)$ & \\
\hline \multirow[t]{2}{*}{3} & $\mu^{(1)}=\left(\begin{array}{l}1 \\
2\end{array}\right.$ & $\Sigma^{(1)}=\left(\begin{array}{cc}1 & 0 \\
& 0.25\end{array}\right)$ & - \\
\hline & $\mu^{(2)}=\left(\begin{array}{l}1 \\
2\end{array}\right.$ & $\Sigma^{(1)}=\left(\begin{array}{cc}1 & 0 \\
& 0.25\end{array}\right)$ & \\
\hline
\end{tabular}

sampling method and the $\mathrm{QB}$, all components have equal prior distributions. The Gibbs sampler is allowed to have 100 iterations while both EM and QB are either stopped if the change in likelihood between two consecutive iterations is less than $\exp (-6)$ or if they reach maximum number of 100 iterations.

As the obtained results reported in Table 6.2 present, all three methods have reasonable accuracy for the first scenario while Gibbs sampler outperforms the others in cost of much higher convergence time. Figure 6.3 presents the convergence rate of the estimated parameters $\mu_{d}^{(k)}$ of the three algorithms for one sample with $d$ denoting the dimension and $k$ the component number. As the Figures suggest, $\mathrm{QB}$ and $\mathrm{EM}$ both have relatively fast convergence rate while Gibbs sampler requires more iterations.

In the second scenario where data samples are simulated such that $1 \%$ of the data is outlier, the QB algorithm outperforms the two others in the MSE sense. In the scenario where the underlying distribution is a single multivariate Gaussian, both $\mathrm{QB}$ and $\mathrm{EM}$ can appropriately estimate the unknown parameters.

To further validate the QB algorithm, its likelihood value over the iterations is also presented in Figure 6.4. As the figure suggests, the likelihood value is monotonically increasing until convergence to the estimated optimal value.

\subsubsection{Experimental data}

In this section, the performance of the QB algorithm is evaluated on the real experimental data. TOA measurement errors corresponding to the explained data collection campaign in Section 5.2.2 are computed relative to the logged GPS positions. That is, at each time instant, knowing the location of the BS, the true distance to the UE is calculated using GPS positions and then subtracted from 
Table 6.2: MSE of estimated parameters corresponding to the three scenarios described in 6.1 over 500 Monte Carlo simulations.

\begin{tabular}{|c|c|c|c|c|}
\hline \multirow{2}{*}{ Scenario } & \multirow{2}{*}{ Parameter } & \multicolumn{3}{|c|}{ MSE } \\
\hline & & QB & EM & Gibbs \\
\hline \multirow{6}{*}{1} & $\hat{\mu}^{(1)}$ & 0.0732 & 0.2257 & 0.0048 \\
\hline & $\hat{\boldsymbol{\mu}}^{(2)}$ & 0.0753 & 0.2276 & 0.0049 \\
\hline & $\hat{\Sigma}^{(1)}$ & 0.1566 & 0.5172 & 0.0048 \\
\hline & $\hat{\Sigma}^{(2)}$ & 0.1575 & 0.5102 & 0.0051 \\
\hline & $\hat{p}\left(s_{n}=1 \mid \boldsymbol{e}, \psi\right)$ & 0.0004 & 0.0103 & 0.0001 \\
\hline & $\hat{\psi}$ & 0.4630 & 1.4911 & 0.0196 \\
\hline \multirow{6}{*}{2} & $\hat{\mu}^{(1)}$ & 0.0008 & 0.0087 & 0.0052 \\
\hline & $\hat{\mu}^{(2)}$ & 1.6160 & 0.7774 & 14.6624 \\
\hline & $\hat{\Sigma}^{(1)}$ & 0.0012 & 0.0123 & 0.0051 \\
\hline & $\hat{\Sigma}^{(2)}$ & 10.3177 & 15.5936 & 43.4677 \\
\hline & $\hat{p}\left(s_{n}=1 \mid \boldsymbol{e}, \psi\right)$ & 0.0003 & 0.0005 & 0.0001 \\
\hline & $\hat{\psi}$ & 11.9360 & 16.3925 & 58.1404 \\
\hline \multirow{5}{*}{3} & $\hat{\mu}^{(1)}$ & 0.0035 & 0.0325 & 0.0129 \\
\hline & $\hat{\boldsymbol{\mu}}^{(2)}$ & 0.0421 & 0.0874 & 37.9156 \\
\hline & $\hat{\Sigma}^{(1)}$ & 0.0026 & 0.0130 & 0.0065 \\
\hline & $\hat{\Sigma}^{(2)}$ & 0.0157 & 0.0302 & 53.6914 \\
\hline & $\hat{\psi}$ & 0.0639 & 0.1631 & 91.6264 \\
\hline
\end{tabular}

the TOA measurement to obtain TOA errors.

There exists algorithms such as a modified version of EM introduced in [121] or the adaptive model proposed in [128] that can be applied to jointly estimate $k$ and parameters. However, in this thesis, the goal is to model the underlying distribution of the TOA errors with a Gaussian mixture with known number of components. We identify $k$ using the two well-known model selection criteria, Akaike's Information criterion (AIC) and Bayesian Information criterion (BIC) given by

$$
\begin{gathered}
A I C(k)=-2 \log p\left(\boldsymbol{e}_{1}, \ldots, \boldsymbol{e}_{N} \mid \psi\right)+2 J(k) \\
B I C(k)=-2 \log \left(\boldsymbol{e}_{1}, \ldots, \boldsymbol{e}_{N} \mid \psi\right)+J(k) \log N .
\end{gathered}
$$

The two criteria are computed using the QB method for $k=1,2, \ldots, 10$ and the results are presented in Figure 6.5. For both BSs, according to the values of the information criteria, the underlying distribution can be modeled using a 3-component Gaussian mixture.

The estimated parameters for the one-dimensional Gaussian mixtures using all three algorithms introduced in Section 6.1 are reported in Table 6.3. It must be noted that in this one-dimensional case all the multivariate distributions should be changed to their univariate counterparts. For instance, the Wishart distribution (6.22) is changed to the Gamma distribution, hence $\left(\sigma^{(k)}\right)^{-2} \sim \Gamma\left(\alpha^{(k)}, \beta^{(k)}\right)$ 

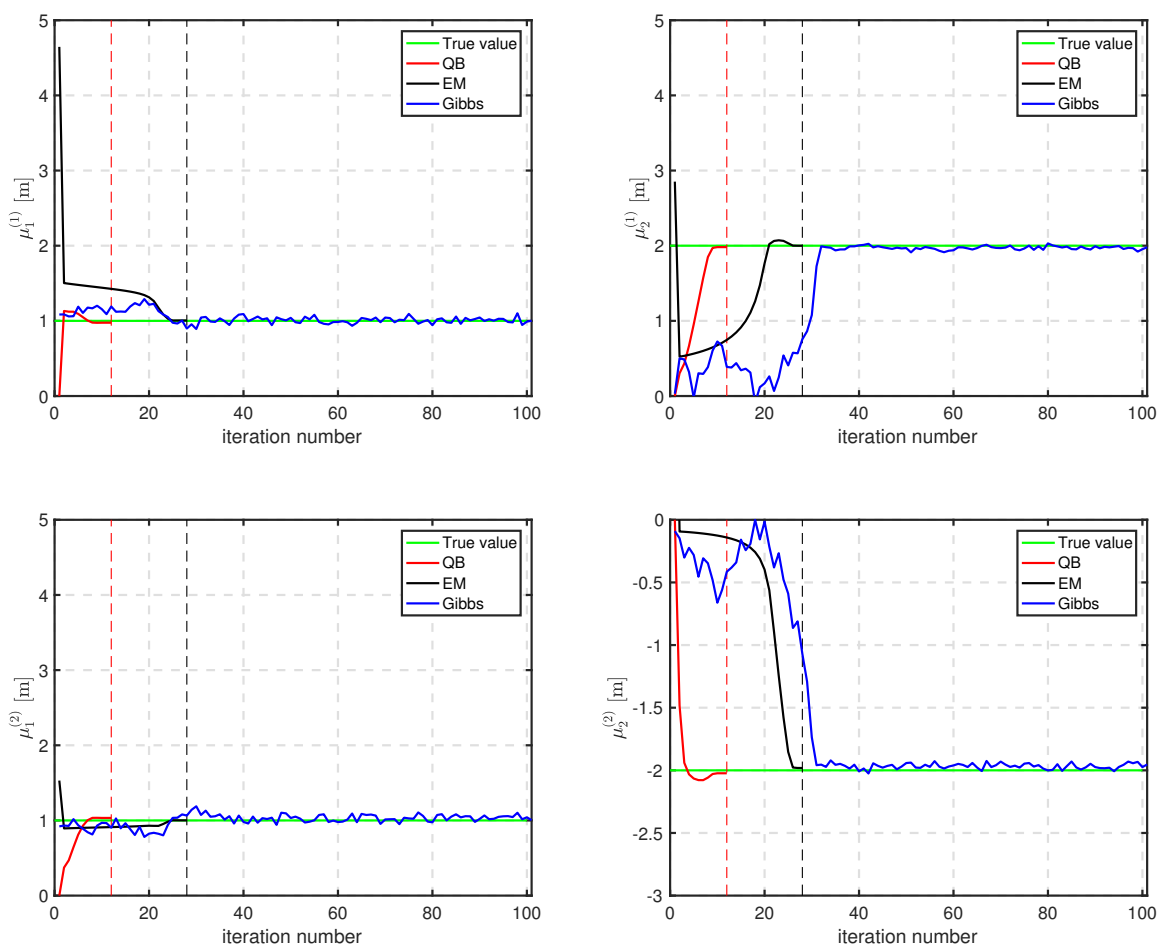

Figure 6.3: Convergence rate of $Q B, E M$, and Gibbs sampling algorithms for estimating parameters $\mu_{d}^{(k)}$ with $k$ denoting the component and $d$ the dimension corresponding to scenario 1 in Table 6.1. The true values are marked with green. The iteration number at which $Q B$ and EM algorithms terminated are marked with red and black vertical dashed lines, respectively.

with $2 \alpha^{(k)}=a^{(k)}+1$ and $2 \beta^{(k)}=b^{(k)}$. The conditional prior for $\mu$ is also given by a uni-variate Normal distribution $\mu^{(k)} \sim \mathcal{N}\left(m^{(k)},\left(\sigma^{(k)}\right)^{2} / v^{(k)}\right)$ with $v^{(k)}=c^{(k)}$.

Table 6.3: Estimated paramters of the 3-component, 1-dimensional, Gaussian mixture fitted to the TOA measurement dataset using QB, EM, and Gibbs sampling algorithms.

\begin{tabular}{l|c|ccc|ccc|cccc}
\hline \multirow{2}{*}{ BS } & \multirow{2}{*}{ Comp } & \multicolumn{3}{|c|}{ QB } & \multicolumn{3}{c|}{ EM } & \multicolumn{3}{c}{ Gibbs } \\
\cline { 3 - 12 } & & $\hat{\mu}$ & $\hat{\sigma}^{2}$ & $\hat{\pi}$ & $\hat{\mu}$ & $\hat{\sigma}^{2}$ & $\hat{\pi}$ & $\hat{\mu}$ & $\hat{\sigma}^{2}$ & $\hat{\pi}$ \\
\hline \multirow{3}{*}{ Serving } & 1 & -0.872 & 29.548 & 0.583 & 1.449 & 59.952 & 0.775 & -0.7 & 28.551 & 0.6 \\
& 2 & 15.542 & 62.476 & 0.404 & 19.557 & 60.77 & 0.188 & 16.12 & 50.157 & 0.3999 \\
& 3 & 0.2142 & 992.026 & 0.013 & 26.572 & 84.396 & 0.037 & 1.1 & 1140 & 0.0001 \\
\hline \multirow{3}{*}{ Neighboring } & 1 & 3.0691 & 33.361 & 0.627 & 1.3232 & 27.5096 & 0.832 & 2.888 & 33.780 & 0.727 \\
& 2 & 24.193 & 246.872 & 0.308 & 14.9358 & 82.6445 & 0.142 & 24.753 & 223.156 & 0.206 \\
& 3 & -2.856 & 2.862 & 0.065 & 45.6678 & 64.8427 & 0.026 & -2.397 & 2.957 & 0.067 \\
\hline
\end{tabular}

The two components with mean values close to zero can be interpreted as 


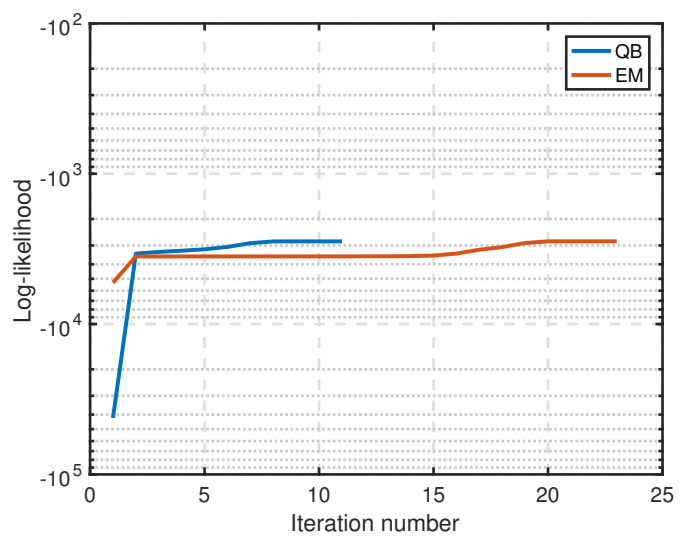

Figure 6.4: The log-likelihood values over iterations of $Q B$ and $E M$ algorithms.

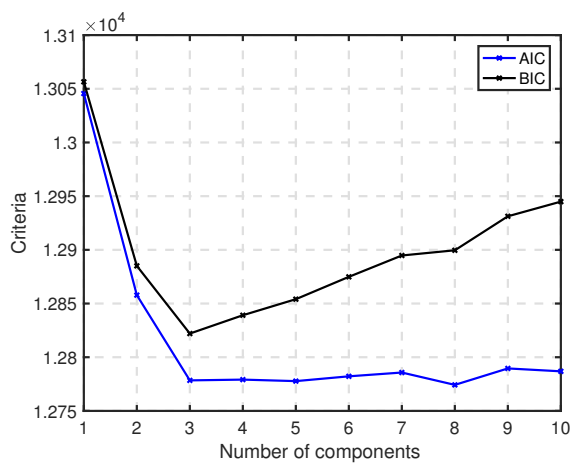

(a) Serving BS.

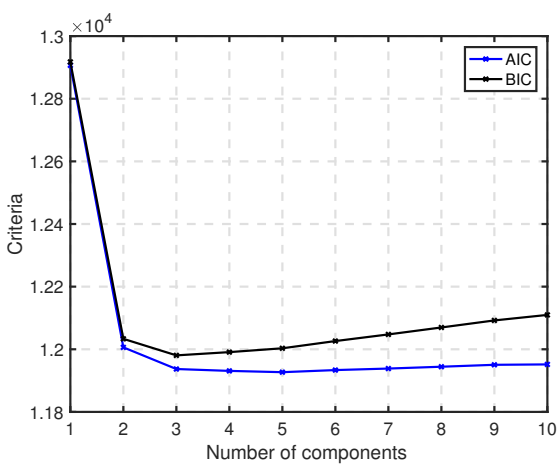

(b) Neighboring BS.

Figure 6.5: Information criteria values given by the $Q B$ algorithm for $k=$ $1, \ldots, 10$ for both serving and neighboring BSs.

measurement noise and multipath propagation errors. As stated in Section 1.2, these two sources of error should have close-to-zero means. The third component, for both BSs, has a larger mean value further away from the origin. This can be interpreted as the NLOS error whose variance is larger than measurement noise variance. While both QB and Gibbs sampler estimated two components with means close to zero, EM estimates one component with small mean and two components with larger mean values. The NLOS component is estimated by all three algorithms with almost similar statistics for the serving BS. However, in case of the neighboring BS, QB and Gibbs sampler estimates are very similar while EM estimates NLOS component closer to the origin with much less variance.

Figure 6.6 illustrates the histogram of the errors of the serving and neighbor- 
ing BSs where the $y$-axis is normalized by density. The Gaussian mixture PDF parameterized by the estimated parameters reported in Table 6.3 are also illustrated in Figure 6.6. As it can be observed from Figure 6.6b, for the neighboring $\mathrm{BS}, \mathrm{QB}$ and Gibbs sampler capture the measurement noise and multipath propagation errors better than EM.

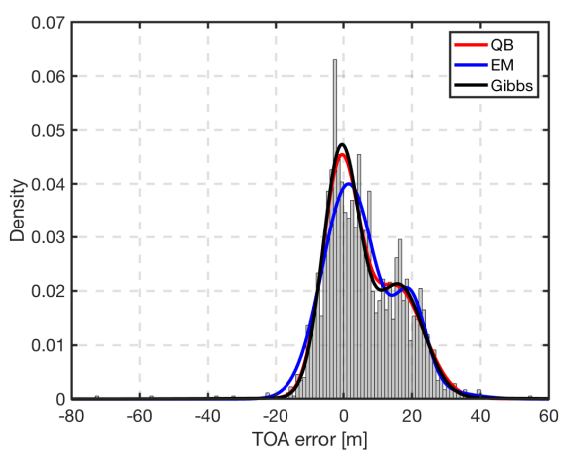

(a) Serving BS.

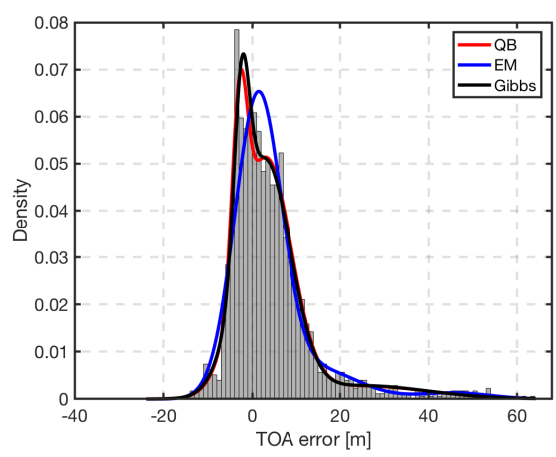

(b) Neighboring BS.

Figure 6.6: Histogram of TOA measurement error of the serving, and neighboring BSs involved in the data collection experiment performed in Kista area, Sweden. The bin width of histogram is $1 \mathrm{~m}$. The Gaussian mixture PDF parameterized by the estimated values reported in Table 6.3 is marked with red.

In order to further evaluate the fit of the estimated parameters, Figure 6.7 compares the empirical CDF of each dataset with the parametric CDF computed by the estimated parameters of the three algorithms. The empirical CDF is obtained from MATLAB built-in function. For errors above $10 \mathrm{~m}$ the empirical CDF and parametric $\mathrm{CDF}$ corresponding to all the three algorithms have almost equal values. However, around $60 \%$ of the times, error terms are below $10 \mathrm{~m}$. In such cases, Gibbs sampler and QB outperform EM, specially for the neighboring BS. 


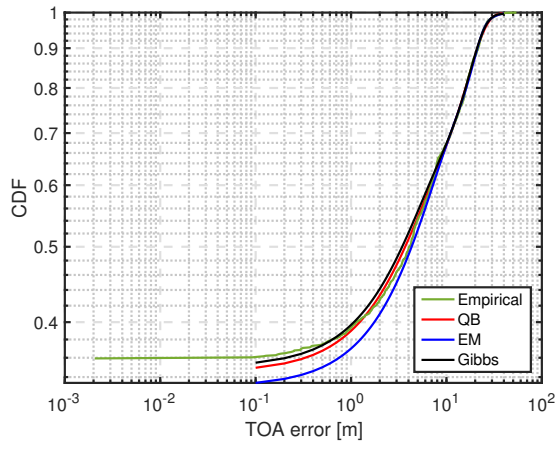

(a) Serving BS.

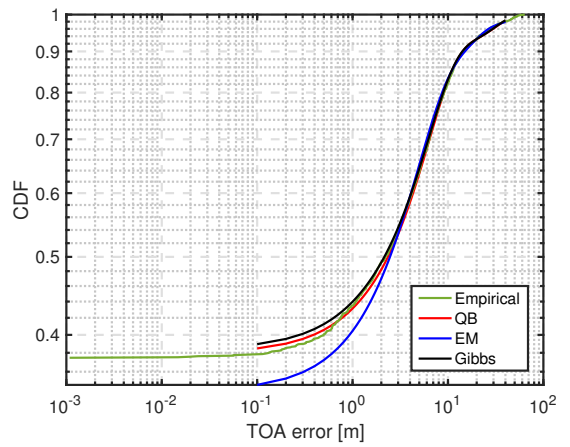

(b) Neighboring BS.

Figure 6.7: Empirical CDF (marked with green), parametric CDF using $Q B$ estimates (marked with red), EM estimates (marked with blue), and Gibbs sampler estimates (marked with black). 



\section{Concluding Remarks and Future Work}

The initial part of this thesis was devoted to a survey of the state of the art in radio network positioning and introduced a general positioning framework and system models. The main contribution of the remainder of the thesis was then to study positioning in radio cellular networks using timing-based measurements. In particular, we evaluated the potential of timing-based positioning in two different scenarios:

- OTDOA positioning in NBIOT systems

- Fusion of TOF and TDOA for 3GPP LTE positioning using measurements collected from two BSs

Finally, initial results of TOA measurement error modeling in presence of NLOS components have been reported. In the following subsections, concluding remarks and possible future works on each of the contributions are provided.

\subsection{State of the art in radio network positioning}

The purpose of this survey was to describe the over-all picture of how state of the art is organized today (see Figure 2.1), recent advances in how the fundamental measurements are computed in recent standards, and pointing out new trends.

The survey presented in this thesis gives the fusion community a fast way into the state-of-the-art in network positioning. It gives an overall picture of the field, the fundamental measurements available, and its organization. This allows for creating relevant simulations and performance analyses, and indicates directions for new research contributions. 


\subsection{Performance of OTDOA positioning in NBIOT systems}

To fulfill different requirements of IOT positioning, 3GPP standardization evolved towards NBIOT systems with enhanced positioning support. The recent 3GPP Release 14 introduces new positioning reference signals with configurable downlink transmission schemes tailored for the narrower system bandwidth. While extensive studies have been performed on timing-based positioning for the legacy LTE systems, NBIOT systems have not been treated equally. This thesis evaluated the performance of localization in NBIOT systems in terms of the horizontal positioning accuracy in a simulation study. The UE assisted OTDOA positioning method was tested to take full advantage of the enhanced NPRS. Simulations accounted for imperfections in wireless channels by considering tapped-delay channel models; EPA and ETU. The positioning performance of both channel models was evaluated for both static and dynamic cases. In the static case, it was assumed that the UE detects at least three and up to six unique BSs. In the dynamic case, an RSTD reporting budget was defined and the effect of the budget on the device tracking performance in NBIOT systems was evaluated.

One possible extension is to derive the theoretical performance limit of NBIOT positioning. Additionally, algorithms more robust against measurement outliers can be developed and compared against the theoretical lower bounds. For example, in a filtering context with a decent prior, gating can be applied, as a simple alternative to order statistics. A second alternative is to formulate the RSTD reporting budget in terms of a mixed integer optimization problem. For instance, given a certain lowest acceptable accuracy threshold, the developed algorithm should adaptively change the number of reported BSs and the reporting interval.

\subsection{Fusion of TDOA and TOF for positioning with two involved BSS}

Different aspects of timing-based 2D positioning in LTE systems when at least three base stations are detected by the UE have already been studied in the literature. This thesis considered the scenario in which only two BSs, one serving and one neighboring, could be detected. Fusion of the TOF measurement of the serving and TDOA measurement of the serving relative to the neighboring BSs has been studied.

The limited number of detected BSs results in ambiguity in the position of the UE. A framework based on a bank of Kalman filters was proposed to deal with the ambiguity in the estimates. Lower bounds on the estimation accuracy were then compared to the obtained RMSE in a simulation study. To further evaluate the developed filtering method, it was tested on real TOA measurements. The achieved results for both the simulated and real data indicated good performance of the introduced method.

One possibility for future work is to extend the filter bank solution with a 
smoothing stage to further increase the estimation accuracy. Additionally, using the geometrical distance for finding the serving BS could also be improved by defining a more relevant handover criterion. The simulation study could also be further extended to be more realistic by adding NLOS imperfections to the measurement errors.

\subsection{TOA error modeling in presence of NLOS components}

The first step towards developing a timing-based position estimation method which is robust against NLOS effects, is to estimate the PDF of the errors. This thesis has set the stage for a robust method by modeling TOA errors in terms of Gaussian mixtures. The number of components in the mixture model was first estimated by investigating AIC and BIC. Given the prior knowledge on the number of components, the maximum likelihood based EM algorithm, pure Bayesian Gibbs sampler, and the quasi-Bayesian algorithms were defined and compared to each other in a simulation study with known true parameter values. Then, they all were tested on real TOA measurements obtained through data collection experiments performed in LTE network to estimate the Gaussian mixture PDF components.

A future research direction is to derive the distribution of TDOA measurement errors and then estimate its parameters using the QB algorithm. This allows us to model both the TOA and TDOA errors in the applications introduced in this thesis. The next step would then be to develop an iterative algorithm that jointly estimates the error PDF and the position. 



\section{Bibliography}

[1] Wireless E911 location accuracy requirements. Technical report, Federal Communications Commission, 2015. Cited on page 19.

[2] 3GPP TS 05.05. 3rd generation partnership project;radio transmission and reception, 1999. Cited on page 38 .

[3] 3GPP TS 22.368. Service requirements for machine-type communications (MTC), December 2014. Cited on page 35.

[4] 3GPP TS 36.101. User equipment (UE) radio transmission and receptio. Cited on page 38 .

[5] 3GPP TS 36.104. Base station (BS) radio transmission and reception. Cited on page 38 .

[6] 3GPP TS 36.211. Evolved Universal Terrestrial Radio Access (E-UTRA); physical channels and modulation. Cited on pages 13 and 37.

[7] Nokia Motorola Rohde \& Schwarz 3GPP, Ericsson. LTE channel models and simulations. Technical report, RAN4-38, 2006. Cited on page 38.

[8] Nokia Motorola Rohde \& Schwarz 3GPP, Ericsson. Proposal for LTE channel models. Technical report, RAN4-43, 2007. Cited on page 38.

[9] 3GPP TR 36.355. Evolved Universal Terrestrial Radio Access (E-UTRA); LTE Positioning Protocol (LPP), Release 12. Cited on page 24.

[10] N. M. Laird A. P. Dempster and D. B Rubin. Maximum-likelihood from incomplete data via the EM algorithm. Journal of the Royal Statistical Society, B39:1-38, 1977. Cited on pages 70 and 76.

[11] M. Agiwal, A. Roy, and N. Saxena. Next generation 5G wireless networks: A comprehensive survey. IEEE Communications Surveys Tutorials, 18(3): 1617-1655, 2016. Cited on page 23.

[12] B. Alavi and K. Pahlavan. Bandwidth effect on distance error modeling for indoor geolocation. In Proc. of 14th Annual International Symposium on 
Personal Indoor and Mobile Radio Communications (PIMRC), pages 21982202, Beijing, China, September 2003. Cited on page 4.

[13] B. Alavi and K. Pahlavan. Modeling of the distance error for indoor geolocation. In Proc. of IEEE Wireless Communications and Networking, pages 668-672, New Orleans, USA, March 2003. Cited on page 4.

[14] B. Alavi and K. Pahlavan. Modeling of the TOA-based distance measurement error using UWB indoor radio measurements. IEEE Communications Letters, 10(4):275-277, April 2006. Cited on page 4.

[15] I. Arasaratnam and S. Haykin. Cubature Kalman filters. IEEE Transactions on Automatic Control, 54(6):1254-1269, June 2009. Cited on page 30.

[16] I. Arasaratnam, S. Haykin, and R. J. Elliott. Discrete-time nonlinear filtering algorithms using Gauss-Hermite quadrature. Proceedings of the IEEE, 95(5):953-977, May 2007. Cited on page 30.

[17] I. Arasaratnam, S. Haykin, and T. R. Hurd. Cubature Kalman filtering for continuous-discrete systems: Theory and simulations. IEEE Transactions on Signal Processing, 58(10):4977-4993, October 2010. Cited on page 30.

[18] D. Astely, E. Dahlman, G. Fodor, S. Parkvall, and J. Sachs. LTE release 12 and beyond. IEEE Communications Magazine, 51(7):154-60, July 2013. Cited on page 22 .

[19] B. M. Bell and F. W. Cathey. The iterated Kalman filter update as a GaussNewton method. IEEE Transactions on Automatic Control, 38(2):294-297, February 1993. Cited on page 31.

[20] H. Bensmail, G. Celeux, A. E. Raftery, and C. P. Robert. Inference in modelbased cluster analysis. Statistics and Computing, 7(1):1-10, March 1997. ISSN s. Cited on page 71.

[21] C. M. Bishop. Pattern Recognition and Machine Learning (Information Science and Statistics). Springer-Verlag New York, Inc., 2006. Cited on page 69.

[22] J. J. Caffery. Wireless Location in CDMA Cellular Radio Systems. Kluwer Academic Publishers, 1999. Cited on pages 2 and 18.

[23] J. J. Caffery and G. L. Stuber. Overview of radiolocation in CDMA cellular systems. IEEE Communications Magazine, 36(4):38-45, April 1998. Cited on pages 2 and 11 .

[24] Y. T. Chan and K. C. Ho. A simple and efficient estimator for hyperbolic location. IEEE Transactions on Signal Processing, 42(8):1905-1915, August 1994. Cited on page 2.

[25] C. Y. Chen and W. R. Wu. Three-dimensional positioning for LTE systems. IEEE Transactions on Vehicular Technology, 66(4):3220-3234, Apr. 2017. Cited on page 24 . 
[26] J. C. Chen, C. S. Maa, Y. C. Wang, and J. T. Chen. Mobile position location using factor graphs,. IEEE Communications Letters, 7(9):431-433, September 2003. Cited on page 2 .

[27] P. C. Chen. A non-line-of-sight error mitigation algorithm in location estimation. In Proc. of IEEE International Conference Wireless Communication Networks (WCNC), pages 316-320, New Orleans, LA, USA, September 1999. Cited on page 3.

[28] K. W. Cheung, H. C. So, W. K. Ma, and Y. T. Chan. A constrained least squares approach to mobile positioning: Algorithms and optimality. EURASIP Journal on Advances in Signal Processing, 2006(1):1-23, April 2006. Cited on page 2 .

[29] C. Chong, C. Tan, D. I. Laurenson, S. McLaughlin, M. A. Beach, and A. R. Nix. A new statistical wideband spatio-temporal channel model for 5-GHz band WLAN systems. IEEE Journal on Selected Areas in Communications, 21(2):139-150, February 2003. Cited on page 48.

[30] L. Cong and W. Zhuang. Hybrid TDOA/AOA mobile user location for wideband CDMA cellular systems. IEEE Transactions on Wireless Communications, 1(3):439-447, July 2002. Cited on pages 2 and 48.

[31] L. Cong and W. Zhuang. Non-line-of-sight error mitigation in mobile location. IEEE Transactions on Wireless Communications, 4(2):560-573, March 2005. Cited on page 3.

[32] J. A. del Peral-Rosado, J. A. López-Salcedo, G. Seco-Granados, F. Zanier, and M. Crisci. Evaluation of the LTE positioning capabilities under typical multipath channels. In Proc. of 6th Advanced Satellite Multimedia Systems Conference (ASMS) and 12th Signal Processing for Space Communications Workshop (SPSC), pages 139-146, Vigo, Spain, September 2012. Cited on page 38 .

[33] J. A. del Peral-Rosado, J. A. López-Salcedo, F. Zanier, and M. Crisci. Achievable localization accuracy of the positioning reference signal of 3GPP LTE. In Proc. of 2nd International Conference on Localization and GNSS, pages 1-6, Starnberg, Germany, June 2012. Cited on page 18.

[34] J. Dennis and R. Schnabel. Numerical Methods for Unconstrained Optimization and Nonlinear Equations. Society for Industrial and Applied Mathematics, 1996. Cited on page 27.

[35] J. Diebolt and C. P. Robert. Estimation of finite mixture distributions through Bayesian sampling. Royal Statistical Society, 56(2):363-375, 1994. Cited on page 71.

[36] C. Drane, M. Macnaughtan, and C. Scott. Positioning GSM telephones. IEEE Communications Magazine, 36(4):46-54,59, April 1998. Cited on pages 11 and 18 . 
[37] M. Driusso, C. Marshall, M. Sabathy, F. Knutti, H. Mathis, and F. Babich. Vehicular position tracking using LTE signals. IEEE Transactions on Vehicular Technology, 66(4):3376-3391, Apr. 2017. Cited on page 24.

[38] M. D. Escobar and M. West. Bayesian density estimation and inference using mixtures. American Statistical Association, 90(430):577-588, June 1995. Cited on page 71 .

[39] FCC. Wireless e911 location accuracy requirements, fourth report and order, March 2015. Cited on page 2.

[40] S. Fischer. Observed time difference of arrival (OTDOA) positioning in 3GPP LTE. 2014. Cited on page 36.

[41] R. A. Fisher. On the mathematical foundations of theoretical statistics. Philosophical Transactions of the Royal Society of London A: Mathematical, Physical and Engineering Sciences, 222(594-604):309-368, 1922. Cited on page 58.

[42] R. A. Fisher. Theory of statistical estimation. Mathematical Proceedings of the Cambridge Philosophical Society, 22(5):700-725, 1925. Cited on page 58 .

[43] R. Fletcher. Practical methods of optimization. Wiley, 1987. Cited on page 70.

[44] IEEE Standard for Information technology. Telecommunications and information exchange between systems local and metropolitan area networksspecific requirements - part 11: Wireless LAN medium access control (MAC) and physical layer (PHY) specifications. IEEE Std 802.11-2016 (Revision of IEEE Std 802.11-2012), pages 1-3534, December 2016. Cited on page 21 .

[45] S. Frattasi and F. D. Rosa. Mobile Positioning and Tracking: From Conventional to Cooperative Techniques. John Wiley \& Sons, Ltd, 2017. Cited on pages 15,16 , and 17 .

[46] C. Fritsche and A. Klein. On the performance of mobile terminal tracking in urban GSM networks using particle filters. In Proc. of 17th European Signal Processing Conference, pages 1953-1957, Glasgow, Scotland, August 2009. Cited on page 3 .

[47] C. Fritsche, U. Hammes, A. Klein, and A. M. Zoubir. Robust mobile terminal tracking in NLOS environments using interacting multiple model algorithm. In Proc. of International Conference on Acoustics, Speech and Signal Processing (ICASSP), pages 3049-3052, Taipei, Taiwan, April 2009. Cited on page 3.

[48] N. Garcia, H. Wymeersch, E. G. Larsson, A. M. Haimovich, and M. Coulon. Direct localization for massive MIMO. IEEE Transactions on Signal Processing, 65(10):2475-2487, May 2017. Cited on page 21. 
[49] S. Geman and D. Geman. Stochastic relaxation, Gibbs distributions, and the Bayesian restoration of images. IEEE Transactions on Pattern Analysis and Machine Intelligence, PAMI-6(6):721-741, November 1984. Cited on page 73 .

[50] M. D. Gillette and H. F. Silverman. A linear closed-form algorithm for source localization from time-differences of arrival. IEEE Signal Processing Letters, 15:1-4, January 2008. Cited on page 2.

[51] A. Guerra, F. Guidi, and D. Dardari. Position and orientation error bound for wideband massive antenna arrays. In Proc. of IEEE International Conference on Communication Workshop (ICCW), pages 853-858, June 2015. Cited on page 21.

[52] F. Guidi, A. Guerra, and D. Dardari. Personal mobile radars with Millimeter-Wave massive arrays for indoor mapping. IEEE Transactions on Mobile Computing, 15(6):1471-1484, June 2016. Cited on page 23.

[53] F. Gunnarsson, F. Lindsten, and N. Carlsson. Particle filtering for networkbased positioning terrestrial radio networks. In Proc. of 10th IET conference on Data Fusion Target Tracking (DF TT), pages 1-7, University of Liverpool, UK, April 2014. Cited on pages 14 and 19.

[54] F. Gustafsson. Statistical Sensor Fusion. Professional Publishing House, 2012. Cited on pages 26, 30, 51, and 55 .

[55] F. Gustafsson and F. Gunnarsson. Mobile positioning using wireless networks: possibilities and fundamental limitations based on available wireless network measurements. IEEE Signal Processing Magazine, 22(4):4135, July 2005. Cited on pages 3, 11, and 18.

[56] J. D. Hamilton. A quasi-bayesian approach to estimating parameters for mixtures of normal distributions. Journal of Business and Economic Statistics, 9(1):27-39, January 1991. Cited on page 76.

[57] U. Hammes and A. M. Zoubir. Robust MT tracking based on M-estimation and interacting multiple model algorithm. IEEE Transactions on Signal Processing, 59(7):3398-3409, July 2011. Cited on page 3.

[58] U. Hammes, E. Wolsztynski, and A. M. Zoubir. Robust tracking and geolocation for wireless networks in NLOS environments. IEEE Journal of Selected Topics in Signal Processing, 3(5):889-901, October 2009. Cited on page 3.

[59] D. N. Hatfield. A report on technical and operational issues impacting the provision of wireless enhanced 911 services. Technical report, Federal Communications Commission, 2002. Cited on page 19.

[60] G. Hendeby. Performance and Implementation Aspects of Nonlinear Filtering. PhD thesis, Linköping University, The Institute of Technology, SE58183 Linköping, Sweden, 2008. Cited on page 55. 
[61] Y. Huang, J. Benest, G. W. Elko, and R. M. Mersereati. Real-time passive source localization: a practical linear-correction least-squares approach. IEEE Transactions on Speech and Audio Processing, 9(8):943-956, November 2001. Cited on page 2.

[62] J. M. Huerta and J. Vidal. Mobile tracking using UKF, time measures and LOS-NLOS expert knowledge. In Proc. of International Conference on Acoustics, Speech, and Signal Processing (ICASSP), pages 901-904, Philadelphia, PA, USA, March 2005. Cited on page 3.

[63] A. H. Jazwinski. Stochastic Processes and Filtering Theory. Dover Publications, 2007. Cited on pages 28 and 30.

[64] Y. Jiang, Q. Hu, and D. K. Yang. A novel position estimation method using hybrid TOA and TDOA in AIS. Journal of Convergence Information Technology, 8(5), March 2013. Cited on page 48.

[65] L. Jiangi, W. Jiafu, W. Qinruo, D. Pan, Z Keliang, and Q. Yupeng. A survey on position-based routing for vehicular ad hoc networks. Telecommunication Systems, 62(1):15-30, May 2016. Cited on page 22.

[66] S. J. Julier and J. K. Uhlmann. Unscented filtering and nonlinear estimation. Proceedings of the IEEE, 92(3):401-422, March 2004. Cited on page 31.

[67] A. Kangas, I. Siomina, and T. Wigren. Positioning in LTE. John Wiley \& Sons, Inc., 2011. Cited on page 24.

[68] H. Kaouach, L. Dussopt, J. Lanteri, T. Koleck, and R. Sauleau. Wideband low-loss linear and circular polarization transmit-arrays in V-Band. IEEE Transactions on Antennas and Propagation, 59(7):2513-2523, July 2011. Cited on page 23.

[69] P. Kasebzadeh, C. Fritsche, G. Hendeby, F. Gunnarsson, and F. Gustafsson. Improved pedestrian dead reckoning positioning with gait parameter learning. In Proc. of 19th International Conference on Information Fusion (FUSION), pages 379-385, Heidelberg, Germany, July 2016. Cited on pages 15 and 25.

[70] P. Kasebzadeh, G. Hendeby, C. Fritsche, F. Gunnarsson, and F. Gustafsson. IMU dataset for motion and device mode classification. In Proc. of 8th International Conference on Indoor Positioning and Indoor Navigation (IPIN), pages 1-8, Sapporo, Japan, September 2017. Cited on page 18.

[71] R. Kaune, J. Horst, and W. Koch. Accuracy analysis for TDOA localization in sensor networks. In Proc. of 14th International Conference on Information Fusion, pages 1-8, Chicago, Illinois, USA, July 2011. Cited on page 41 .

[72] S. M. Kay. Fundamentals of Statistical Signal Processing: Estimation Theory. Prentice-Hall, Inc., Upper Saddle River, NJ, USA, 1993. Cited on pages 26 and 59. 
[73] K. Keunecke and G. Scholl. Deriving 2d TOA/TDOA IEEE 802.11 g/n/ac location accuracy from an experimentally verified fading channel model. In Proc. of 4th Indoor Positioning and Indoor Navigation, pages 1-10, Belfort, France, October 2013. Cited on page 18.

[74] S. H. Kong and B. Kim. Error analysis of the OTDOA from the resolved first arrival path in LTE. IEEE Transactions on Wireless Communications, 15(10):6598-6610, Oct. 2016. Cited on page 3.

[75] H. Krim and M. Viberg. Two decades of array signal processing research: the parametric approach. IEEE Signal Processing Magazine, 13(4):67-94, July 1996. Cited on pages 14,21 , and 48 .

[76] Y. Amizur L. Banin, U. Schatzberg. WiFi FTM and map information fusion for accurate positioning. In Proc. of 7th Indoor Positioning and Indoor Navigation (IPIN), Alcalá de Henares, Spain, October 2016. Cited on page 21.

[77] C. Lass, D. A. Medina, I. D. H. Pinzón, and R. Ziebold. Methods of robust snapshot positioning in Multi-Antenna systems for inland water applications. In Proc. of the European Navigation Conference, Helsinki, Finnland, May 2016. Cited on page 28.

[78] M. Lavine and M. West. A Bayesian method for classification and discrimination. Canadian Journal of Statistics, 20(4):451-461, December 1992. Cited on page 73.

[79] E. L. Lehmann. Theory of Point Estimation. John Wiley and sons, 1983. Cited on page 58.

[80] X. Rong Li and V. P. Jilkov. Survey of maneuvering target tracking. Part I. dynamic models. IEEE Transactions on Aerospace and Electronic Systems, 39(4):1333-1364, October 2003. Cited on pages 32 and 33.

[81] Z. Li, D. C. Dimitrova, D. H. Raluy, and T. Braun. TDOA for narrow-band signal with low sampling rate and imperfect synchronization. In Proc. of the 7th IFIP Wireless and Mobile Networking Conference (WMNC), pages 1-8, Vilamoura, Portugal, May 2014. Cited on page 3.

[82] J. F. Liao and B. S. Chen. Robust mobile location estimator with NLOS mitigation using interacting multiple model algorithm. IEEE Transactions on Wireless Communications, 5(11):3002-3006, November 2006. Cited on page 3 .

[83] X. Lin, J. Bergman, F. Gunnarsson, O. Liberg, S. M. Razavi, H. S. Razaghi, H. Rydn, and Y. Sui. Positioning for the internet of things: A 3GPP perspective. IEEE Communications Magazine, 55(12):179-185, December 2017. Cited on pages 35 and 36. 
[84] G. Liu, M. Iwai, Y. Tobe, D. MAtekenya, K. M. A. Hossain, M. Ito, and K. Sezaki. Beyond horizontal location context: Measuring elevation using smartphone's barometer. In Proc. of ACM International Joint Conference on Pervasive and Ubiquitous Computing, pages 459-468, Seattle, Washington, September 2014. Cited on page 20.

[85] U. Makov and A. Smith. A quasi-bayes unsupervised learning procedure for priors. IEEE Transactions on Information Theory, 23(6):761-764, November 1977. Cited on page 75.

[86] U. E. Makov and A. F. M. Smith. Quasi-bayes procedures for unsupervised learning. In Conf. on Decision and Control including the 15th Symposium on Adaptive Processes, pages 408-412, December 1976. Cited on page 75.

[87] A. Mallat, J. Louveaux, and L. Vandendorpe. UWB based positioning in multipath channels: CRBs for AOA and for hybrid TOA-AOA based methods. In Proc. of IEEE International Conference on Communications, pages 5775-5780, Glasgow, Scotland, June 2007. Cited on page 48.

[88] M. McGuire, K. N. Plataniotis, and A. N. Venetsanopoulos. Data fusion of power and time measurements for mobile terminal location. IEEE Transactions on Mobile Computing, 4(2):142-153, March 2005. Cited on page 3.

[89] G. McLachlan and T. Krishnan. The EM Algorithm and Extensions. Wiley, 1996. Cited on page 70.

[90] J. Medbo, I. Siomina, A. Kangas, and J. Furuskog. Propagation channel impact on LTE positioning accuracy: A study based on real measurements of observed time difference of arrival. In Proc. of 20th IEEE International Symposium on Personal, Indoor and Mobile Radio Communications, pages 2213-2217, Westin Toyko, Toyko, Japan, September 2009. Cited on pages 2,64 , and 65 .

[91] X. L. Meng and D. Van Dyk. The EM algorithm-an old folk-song sung to a fast new tune. Royal Statistical Society, 59(3):511-567, 1997. Cited on page 70 .

[92] C. Mensing and S. Plass. TDoA positioning based on factor graphs. In Proc. of 17th International Symposium on Personal, Indoor and Mobile Radio Communications, pages 1-5, Helsinki, Finland, September 2006. Cited on page 2 .

[93] A. F. Molisch. Wireless Communications. Wiley Publishing, 2011. Cited on page 38 .

[94] K. Muralidharan, A. J. Khanand Misra, B. Archan, K. Rajesh, and S. Agarwal. Barometric phone sensors: More hype than hope! In Proc. of 15th Workshop on Mobile Computing Systems and Applications, pages 12:112:6, Santa Barbara, California, February 2014. Cited on page 20. 
[95] K. P. Murphy. Machine Learning: A Probabilistic Perspective. The MIT Press, 2012. Cited on pages 70, 71, and 76.

[96] D. Niculescu and B. Nath. Ad hoc positioning system (APS) using AOA. In Proc. of IEEE INFOCOM. 22nd Annual Joint Conference of the IEEE Computer and Communications Societies, pages 1734-1743, March 2003. Cited on page 22 .

[97] J. Nocedal and S. J. Wright. Numerical Optimization. Springer, 2006. Cited on page 27.

[98] Y. Qi, H. Kobayashi, and H. Suda. Analysis of wireless geolocation in a non-line-of-sight environment. IEEE Transactions on Wireless Communications, 5(3):672-681, March 2006. Cited on page 48.

[99] K. Radnosrati, F. Gunnarsson, and F. Gustafsson. New trends in radio network positioning. In Proc. of 18th International Conference on Information Fusion (Fusion), pages 492-498, Washington, D.C., USA, July 2015. Cited on pages 3 and 12 .

[100] K. Radnosrati, C. Fritsche, G. Hendeby, F. Gunnarsson, and F. Gustafsson. Fusion of TOF and TDOA for 3GPP positioning. In Proc. of 19th International Conference on Information Fusion (FUSION), pages 1454-1460, Heidelberg, Germany, July 2016. Cited on pages 6 and 50.

[101] K. Radnosrati, G. Hendeby, C. Fritsche, F. Gunnarsson, and F. Gustafsson. Performance of OTDOA positioning in narrowband IoT systems. In Proc. of 28th Annual International Symposium on Personal, Indoor, and Mobile Radio Communications (PIMRC), pages 1-7, Montreal, Canada, October 2017. Cited on pages $5,37,40,42,43$, and 44 .

[102] S. Richardson and P. J. Green. On Bayesian analysis of mixtures with an unknown number of components. Royal Statistical Society, 59(4):731-792, 1997. Cited on page 71 .

[103] A. Rico-Alvarino, M. Vajapeyam, H. Xu, X. Wang, Y. Blankenship, J. Bergman, T. Tirronen, and E. Yavuz. An overview of 3GPP enhancements on machine to machine communications. IEEE Communications Magazine, 54(6):14-21, June 2016. Cited on page 35.

[104] S. Rosati, K. Krużelecki, G. Heitz, D. Floreano, and B. Rimoldi. Dynamic routing for flying ad hoc networks. IEEE Transactions on Vehicular Technology, 65(3):1690-1700, March 2016. Cited on page 22.

[105] F. Rusek, D. Persson, B. K. Lau, E. G. Larsson, T. L. Marzetta, O. Edfors, and F. Tufvesson. Scaling up MIMO: Opportunities and challenges with very large arrays. IEEE Signal Processing Magazine, 30(1):40-60, January 2013. Cited on page 21. 
[106] H. Rydén, S. M. Razavi, F. Gunnarsson, S. M. Kim, M. Wang, Y. Blankenship, A. Grövlen, and Å. Busin. Baseline performance of LTE positioning in 3GPP 3D MIMO indoor user scenarios. In Proc. of the International Conference on Location and GNSS (ICL-GNSS), pages 1-6, Gothenburg, Sweden, Jun. 2015. Cited on pages 3, 5, 39, and 40.

[107] S. Särkkä. Bayesian Filtering and Smoothing. Cambridge University Press, New York, NY, USA, 2013. Cited on pages 28, 30, and 51.

[108] V. Savic and E. G. Larsson. Fingerprinting-based positioning in distributed massive MIMO systems. In Proc. of 82nd IEEE Vehicular Technology Conference (VTC), pages 1-5, Boston, USA, September 2015. Cited on page 21.

[109] A. H. Sayed, A. Tarighat, and N. Khajehnouri. Network-based wireless location: challenges faced in developing techniques for accurate wireless location information. IEEE Signal Processing Magazine, 22(4):24-40, July 2005. Cited on page 11.

[110] A. Shahmansoori, G. E. Garcia, G. Destino, G. Seco-Granados, and $\mathrm{H}$. Wymeersch. Position and orientation estimation through millimeterwave MIMO in 5G systems. IEEE Transactions on Wireless Communications, 17(3):1822-1835, March 2018. Cited on page 23.

[111] H. Shariatmadari, R. Ratasuk, S. Iraji, A. Laya, T. Taleb, R Jäntti, and A. Ghosh. Machine-type communications: current status and future perspectives toward 5G systems. IEEE Communications Magazine, 53(9):1017, September 2015. Cited on page 35.

[112] Y. Shen and M. Z. Win. On the accuracy of localization systems using wideband antenna arrays. IEEE Transactions on Communications, 58(1): 270-280, January 2010. Cited on page 48.

[113] A. Smith and U. Makov. Unsupervised learning for signal versus noise. IEEE Transactions on Information Theory, 27(4):498-500, July 1981. Cited on page 75 .

[114] G. L. Smith, S. F. Schmidt, and L. A. McGee. Application of Statistical Filter Theory to the Optimal Estimation of Position and Velocity on Board a Circumlunar Vehicle. National Aeronautics and Space Administration, 1962. Cited on page 30.

[115] H. C. So, Y. T. Chan, and F. K. W. Chan. Closed-form formulae for timedifference-of-arrival estimation. IEEE Transactions on Signal Processing, 56(6):2614-2620, June 2008. Cited on page 2.

[116] T. B. Sorensen, P. E. Mogensen, and F. Frederiksen. Extension of the ITU channel models for wideband (OFDM) systems. In Proc. of 62nd Vehicular Technology Conference (VTC), pages 392-396, Stockholm, Sweden, September 2005. Cited on page 38. 
[117] G. Sun, J. Chen, W. Guo, and K. J. R. Liu. Signal processing techniques in network-aided positioning: a survey of state-of-the-art positioning designs. IEEE Signal Processing Magazine, 22(4):12-23, July 2005. Cited on page 11.

[118] J. Talvitie, M. Valkama, G. Destino, and H. Wymeersch. Novel algorithms for high-accuracy joint position and orientation estimation in $5 \mathrm{G}$ mmWave systems. In Proc. of IEEE Globecom Workshops (GC Wkshps), pages 1-7, Singapore, December 2017. Cited on page 23.

[119] ITU-R M.1225 International Telecommunication Union. Guidelines for evaluation of radio transmission technologies for IMT-2000, 1997. Cited on page 38 .

[120] B. D. Van Veen and K. M. Buckley. Beamforming: a versatile approach to spatial filtering. IEEE ASSP Magazine, 5(2):4-24, April 1988. Cited on page 48 .

[121] H. X. Wang, B. Luo, and Q. B. Zhang. Estimation for the number of components in a mixture model using stepwise split-and-merge EM algorithm. Pattern Recognition Letters, 25(16):1799-1809, December 2004. Cited on page 81 .

[122] Y. P. E. Wang, X. Lin, A. Adhikary, A. Grovlen, Y. Sui, Y. Blankenship, J. Bergman, and H. S. Razaghi. A primer on 3GPP narrowband internet of things. IEEE Communications Magazine, 55(3):117-123, Mar. 2017. Cited on pages 24 and 35 .

[123] M. Wax, T.J. Shan, and T. Kailath. Location and the spectral density estimation of multiple sources. Technical Report AFOSR-TR-83-0323, DTIC, Fort Belvoir, VA, USA, 1982. Cited on page 21.

[124] S. Wu, D. Xu, J. Tan, K. Xu, and H. Wang. Two base station location techniques with adjusted measurements in circular scattering environments. International Journal of Communication Systems, 29(6):1073-1083, September 2016. Cited on page 48.

[125] Y. Wu, D. Hu, M. Wu, and X. Hu. A numerical-integration perspective on Gaussian filters. IEEE Transactions on Signal Processing, 54(8):2910-2921, August 2006. Cited on page 30.

[126] H. Wymeersch, G. Seco-Granados, G. Destino, D. Dardari, and F. Tufvesson. $5 \mathrm{G}$ mmWave positioning for vehicular networks. IEEE Wireless Communications, 24(6):80-86, December 2017. Cited on page 23.

[127] H. Xiao, H. Zhang, Z. Wang, and T. A. Gulliver. An RSSI based DV-hop algorithm for wireless sensor networks. In Proc. of Pacific Rim Conference on Communications, Computers and Signal Processing (PACRIM), pages 1-6, Victoria, B.C., Canada, August 2017. Cited on page 22. 
[128] C. H. Xie, J. Y. Chang, and Y. J. Liu. Estimating the number of components in Gaussian mixture models adaptively for medical image. International Journal for Light and Electron Optics, 124(23):6216-6221, December 2013. Cited on page 81.

[129] W. Xu, M. Huang, C. Zhu, and A. Dammann. Maximum likelihood TOA and OTDOA estimation with first arriving path detection for 3GPP LTE system. Transactions on Emerging Telecommunications Technologies, 27 (3):339-356, 2016. Cited on page 19.

[130] F. Yin and A. M. Zoubir. Robust positioning in NLOS environments using nonparametric adaptive kernel density estimation. In IEEE International Conference on Acoustics, Speech and Signal Processing (ICASSP), pages 3517-3520, Kyoto, Japan, March 2012. Cited on page 3.

[131] F. Yin, C. Fritsche, F. Gustafsson, and A. M. Zoubir. TOA-based robust wireless geolocation and Cramér-Rao lower bound analysis in harsh LOS/NLOS environments. IEEE Transactions on Signal Processing, 61(9):2243-2255, May 2013. Cited on pages 4 and 7.

[132] J. Yin, Q. Wan, S. Yang, and K. C. Ho. A sample and accurate TDOA-AOA localization method using two stations. IEEE Signal Processing Letters, 23 (1):144-148, January 2016. Cited on page 48.

[133] A. Zanella. Best practice in RSS measurements and ranging. IEEE Communications Surveys Tutorials, 18(4):2662-2686, April 2016. Cited on page 18.

[134] Y. Zhao. Overview of 2G LCS technnologies and standards. In 3GPP TSG SA2 LCS Workshop, London, UK, January 2001. Cited on page 18.

[135] Y. Zhao. Standardization of mobile phone positioning for 3G systems. IEEE Communications Magazine, 40(7):108-116, July 2002. Cited on pages 11, 18 , and 19.

[136] D. Zhong and Z. Li. The study of MIMO channel modeling and simulation based on 3GPP LTE. In Proc. of 2nd Consumer Electronics, Communications and Networks (CECNet), pages 3646-3651, Hubei, China, April 2012. Cited on page 38 . 


\section{Licentiate Theses \\ Division of Automatic Control \\ Linköping University}

P. Andersson: Adaptive Forgetting through Multiple Models and Adaptive Control of Car Dynamics. Thesis No. 15, 1983.

B. Wahlberg: On Model Simplification in System Identification. Thesis No. 47, 1985.

A. Isaksson: Identification of Time Varying Systems and Applications of System Identification to Signal Processing. Thesis No. 75, 1986.

G. Malmberg: A Study of Adaptive Control Missiles. Thesis No. 76, 1986.

S. Gunnarsson: On the Mean Square Error of Transfer Function Estimates with Applications to Control. Thesis No. 90, 1986.

M. Viberg: On the Adaptive Array Problem. Thesis No. 117, 1987.

K. Ståhl: On the Frequency Domain Analysis of Nonlinear Systems. Thesis No. 137, 1988.

A. Skeppstedt: Construction of Composite Models from Large Data-Sets. Thesis No. 149, 1988.

P. A. J. Nagy: MaMiS: A Programming Environment for Numeric/Symbolic Data Processing. Thesis No. 153, 1988.

K. Forsman: Applications of Constructive Algebra to Control Problems. Thesis No. 231, 1990.

I. Klein: Planning for a Class of Sequential Control Problems. Thesis No. 234, 1990.

F. Gustafsson: Optimal Segmentation of Linear Regression Parameters. Thesis No. 246, 1990.

H. Hjalmarsson: On Estimation of Model Quality in System Identification. Thesis No. 251, 1990.

S. Andersson: Sensor Array Processing; Application to Mobile Communication Systems and Dimension Reduction. Thesis No. 255, 1990.

K. Wang Chen: Observability and Invertibility of Nonlinear Systems: A Differential Algebraic Approach. Thesis No. 282, 1991.

J. Sjöberg: Regularization Issues in Neural Network Models of Dynamical Systems. Thesis No. 366, 1993.

P. Pucar: Segmentation of Laser Range Radar Images Using Hidden Markov Field Models. Thesis No. 403, 1993.

H. Fortell: Volterra and Algebraic Approaches to the Zero Dynamics. Thesis No. 438, 1994.

T. McKelvey: On State-Space Models in System Identification. Thesis No. 447, 1994.

T. Andersson: Concepts and Algorithms for Non-Linear System Identifiability. Thesis No. 448, 1994.

P. Lindskog: Algorithms and Tools for System Identification Using Prior Knowledge. Thesis No. 456, 1994.

J. Plantin: Algebraic Methods for Verification and Control of Discrete Event Dynamic Systems. Thesis No. 501, 1995.

J. Gunnarsson: On Modeling of Discrete Event Dynamic Systems, Using Symbolic Algebraic Methods. Thesis No. 502, 1995.

A. Ericsson: Fast Power Control to Counteract Rayleigh Fading in Cellular Radio Systems. Thesis No. 527, 1995.

M. Jirstrand: Algebraic Methods for Modeling and Design in Control. Thesis No. 540, 1996.

K. Edström: Simulation of Mode Switching Systems Using Switched Bond Graphs. Thesis No. 586, 1996. 
J. Palmqvist: On Integrity Monitoring of Integrated Navigation Systems. Thesis No. 600, 1997.

A. Stenman: Just-in-Time Models with Applications to Dynamical Systems. Thesis No. 601, 1997.

M. Andersson: Experimental Design and Updating of Finite Element Models. Thesis No. 611, 1997.

U. Forssell: Properties and Usage of Closed-Loop Identification Methods. Thesis No. 641, 1997.

M. Larsson: On Modeling and Diagnosis of Discrete Event Dynamic systems. Thesis No. 648, 1997.

N. Bergman: Bayesian Inference in Terrain Navigation. Thesis No. 649, 1997.

V. Einarsson: On Verification of Switched Systems Using Abstractions. Thesis No. 705, 1998.

J. Blom, F. Gunnarsson: Power Control in Cellular Radio Systems. Thesis No. 706, 1998.

P. Spångéus: Hybrid Control using LP and LMI methods - Some Applications. Thesis No. 724, 1998.

M. Norrlöf: On Analysis and Implementation of Iterative Learning Control. Thesis No. 727, 1998.

A. Hagenblad: Aspects of the Identification of Wiener Models. Thesis No. 793, 1999.

F. Tjärnström: Quality Estimation of Approximate Models. Thesis No. 810, 2000.

C. Carlsson: Vehicle Size and Orientation Estimation Using Geometric Fitting. Thesis No. 840, 2000.

J. Löfberg: Linear Model Predictive Control: Stability and Robustness. Thesis No. 866, 2001.

O. Härkegård: Flight Control Design Using Backstepping. Thesis No. 875, 2001.

J. Elbornsson: Equalization of Distortion in A/D Converters. Thesis No. 883, 2001.

J. Roll: Robust Verification and Identification of Piecewise Affine Systems. Thesis No. 899, 2001.

I. Lind: Regressor Selection in System Identification using ANOVA. Thesis No. 921, 2001.

R. Karlsson: Simulation Based Methods for Target Tracking. Thesis No. 930, 2002.

P.-J. Nordlund: Sequential Monte Carlo Filters and Integrated Navigation. Thesis No. 945, 2002.

M. Östring: Identification, Diagnosis, and Control of a Flexible Robot Arm. Thesis No. 948, 2002.

C. Olsson: Active Engine Vibration Isolation using Feedback Control. Thesis No. 968, 2002.

J. Jansson: Tracking and Decision Making for Automotive Collision Avoidance. Thesis No. 965, 2002.

N. Persson: Event Based Sampling with Application to Spectral Estimation. Thesis No. 981, 2002.

D. Lindgren: Subspace Selection Techniques for Classification Problems. Thesis No. 995, 2002.

E. Geijer Lundin: Uplink Load in CDMA Cellular Systems. Thesis No. 1045, 2003.

M. Enqvist: Some Results on Linear Models of Nonlinear Systems. Thesis No. 1046, 2003.

T. Schön: On Computational Methods for Nonlinear Estimation. Thesis No. 1047, 2003.

F. Gunnarsson: On Modeling and Control of Network Queue Dynamics. Thesis No. 1048, 2003.

S. Björklund: A Survey and Comparison of Time-Delay Estimation Methods in Linear Systems. Thesis No. 1061, 2003. 
M. Gerdin: Parameter Estimation in Linear Descriptor Systems. Thesis No. 1085, 2004.

A. Eidehall: An Automotive Lane Guidance System. Thesis No. 1122, 2004.

E. Wernholt: On Multivariable and Nonlinear Identification of Industrial Robots. Thesis No. 1131, 2004.

J. Gillberg: Methods for Frequency Domain Estimation of Continuous-Time Models. Thesis No. 1133, 2004.

G. Hendeby: Fundamental Estimation and Detection Limits in Linear Non-Gaussian Systems. Thesis No. 1199, 2005.

D. Axehill: Applications of Integer Quadratic Programming in Control and Communication. Thesis No. 1218, 2005.

J. Sjöberg: Some Results On Optimal Control for Nonlinear Descriptor Systems. Thesis No. 1227, 2006.

D. Törnqvist: Statistical Fault Detection with Applications to IMU Disturbances. Thesis No. 1258, 2006.

H. Tidefelt: Structural algorithms and perturbations in differential-algebraic equations. Thesis No. 1318, 2007.

S. Moberg: On Modeling and Control of Flexible Manipulators. Thesis No. 1336, 2007.

J. Wallén: On Kinematic Modelling and Iterative Learning Control of Industrial Robots. Thesis No. 1343, 2008.

J. Harju Johansson: A Structure Utilizing Inexact Primal-Dual Interior-Point Method for Analysis of Linear Differential Inclusions. Thesis No. 1367, 2008.

J. D. Hol: Pose Estimation and Calibration Algorithms for Vision and Inertial Sensors. Thesis No. 1370, 2008.

H. Ohlsson: Regression on Manifolds with Implications for System Identification. Thesis No. 1382, 2008.

D. Ankelhed: On low order controller synthesis using rational constraints. Thesis No. 1398, 2009.

P. Skoglar: Planning Methods for Aerial Exploration and Ground Target Tracking. Thesis No. 1420, 2009.

C. Lundquist: Automotive Sensor Fusion for Situation Awareness. Thesis No. 1422, 2009.

C. Lyzell: Initialization Methods for System Identification. Thesis No. 1426, 2009.

R. Falkeborn: Structure exploitation in semidefinite programming for control. Thesis No. 1430, 2010.

D. Petersson: Nonlinear Optimization Approaches to $\mathcal{H}_{2}$-Norm Based LPV Modelling and Control. Thesis No. 1453, 2010.

Z. Sjanic: Navigation and SAR Auto-focusing in a Sensor Fusion Framework. Thesis No. 1464, 2011.

K. Granström: Loop detection and extended target tracking using laser data. Thesis No. 1465, 2011.

J. Callmer: Topics in Localization and Mapping. Thesis No. 1489, 2011.

F. Lindsten: Rao-Blackwellised particle methods for inference and identification. Thesis No. 1480, 2011.

M. Skoglund: Visual Inertial Navigation and Calibration. Thesis No. 1500, 2011.

S. Khoshfetrat Pakazad: Topics in Robustness Analysis. Thesis No. 1512, 2011.

P. Axelsson: On Sensor Fusion Applied to Industrial Manipulators. Thesis No. 1511, 2011.

A. Carvalho Bittencourt: On Modeling and Diagnosis of Friction and Wear in Industrial Robots. Thesis No. 1516, 2012.

P. Rosander: Averaging level control in the presence of frequent inlet flow upsets. Thesis No. 1527, 2012. 
N. Wahlström: Localization using Magnetometers and Light Sensors. Thesis No. 1581, 2013.

R. Larsson: System Identification of Flight Mechanical Characteristics. Thesis No. 1599, 2013.

Y. Jung: Estimation of Inverse Models Applied to Power Amplifier Predistortion. Thesis No. 1605, 2013.

M. Syldatk: On Calibration of Ground Sensor Networks. Thesis No. 1611, 2013.

M. Roth: Kalman Filters for Nonlinear Systems and Heavy-Tailed Noise. Thesis No. 1613, 2013.

D. Simon: Model Predictive Control in Flight Control Design - Stability and Reference Tracking. Thesis No. 1642, 2014.

J. Dahlin: Sequential Monte Carlo for inference in nonlinear state space models. Thesis No. 1652, 2014.

M. Kok: Probabilistic modeling for positioning applications using inertial sensors. Thesis No. 1656, 2014.

J. Linder: Graybox Modelling of Ships Using Indirect Input Measurements. Thesis No. 1681, 2014.

G. Mathai: Direction of Arrival Estimation of Wideband Acoustic Wavefields in a Passive Sensing Environment. Thesis No. 1721, 2015.

I. Nielsen: On Structure Exploiting Numerical Algorithms for Model Predictive Control. Thesis No. 1727, 2015.

C. Veibäck: Tracking of Animals Using Airborne Cameras. Thesis No. 1761, 2016.

N. Evestedt: Sampling Based Motion Planning for Heavy Duty Autonomous Vehicles. Thesis No. 1762, 2016.

H. Nyqvist: On Pose Estimation in Room-Scaled Environments. Thesis No. 1765, 2016.

Y. Zhao: Position Estimation in Uncertain Radio Environments and Trajectory Learning. Thesis No. 1772, 2017.

P. Kasebzadeh: Parameter Estimation for Mobile Positioning Applications. Thesis No. 1786, 2017. 